\title{
Oral pentoxifylline in the treatment of venous leg ulcers: a meta-analysis
}

\author{
Andrew B Jull
}

A thesis submitted to the Victoria University of Wellington in partial fulfilment of the requirements for the degree of Master of Arts (Applied) in Nursing

Victoria University of Wellington

1999 


\begin{abstract}
Compression bandaging improves rates of healing in people with venous ulceration. Some ulcers appear resistant to compression therapy and may benefit from adjunctive therapy. Pentoxifylline is known to improve circulation, but individual trials have failed to conclusively demonstrate its effectiveness in venous ulceration. The objective of this meta-analysis was to assess the effectiveness of pentoxifylline as an adjunct to compression bandaging in the treatment of venous leg ulcers.
\end{abstract}

The CENTRAL registers of the Cochrane Peripheral Vascular Diseases and Wounds Groups were searched - each register is routinely updated by extensive searches of electronic databases, handsearching of relevant journals and conference proceedings, and contact with product companies and experts in the field. The drug's manufacturer was contacted and the references of review articles and all obtained trials were scrutinised for further citations.

Randomised controlled trials published in any language comparing pentoxifylline and compression with placebo in adult participants with venous ulceration were included. Trials must have reported a meaningful objective outcome (rates of healing, proportions healed, time to healing). Details from eligible trials (independently selected by two reviewers) were extracted and summarised by one reviewer. A second reviewer independently verified extracted data.

Eleven clinical trials were identified. Five trials compared pentoxifylline with placebo (compression standard therapy). Six trials were excluded. Pentoxifylline and compression was more effective than placebo and compression (RR 1.30, 95\% CI 1.10-1.54) and was robust to sensitivity analyses. The greater number of adverse effects (46 reports) occurred in the pentoxifylline group, although this was not significant (RR 1.12, 95\% CI 0.77-1.62); 34 percent of adverse effects were gastrointestinal. Seven of 21 reported withdrawals were for adverse effects.

In conclusion, pentoxifylline appears an effective adjunct to compression bandaging in the treatment of venous ulcers. The absence of cost-effectiveness data suggests it not be employed as a routine adjunct, but it could be considered as for those patients not responding to compression therapy alone. The majority of adverse effects are likely to be tolerated by patients. 


\section{ACKNOWLEDGEMEN'TS}

I wish to thank my co-reviewers, Associate Professor Bruce Arroll, Department of General Practice, Faculty of Medicine and Health Sciences, University of Auckland, and Dr Jill Waters, Geriatrician, A+ Links, Auckland Healthcare Services, for assistance and advice. Without their gracious support, this review could not have been conducted with the necessary degree of rigour. Librarians, and reference librarians in particular, are a group of people I would include on my desert isle should that choice ever arise. Sue Foggin, Lorraine Neilsen, Susan Hope, and Sarak 'Tritt, Reference Librarians, Philson Library, University of Auckland, Alison Cooke and Tricia Binghams, Librarians, Auckland Healthcare Services, always responded magnificently to my frequent requests. Many people helped me when I encountered a problem that seemed insurmountable: Andrea Nelson, Co-ordinator, Cochrane Wound Review Group, University of York, deserves especiai mention for her tolerant, timely advice and rapid response to my frequent pleas; Amanda Henry assisted me with typing during an OOS crisis; Sarah Hetrick, Co-ordinator, Cochrarie Sub-fertility and Dysmenorrhoea Group, University of Auckland, sorted out one of my translation problems with marvellous ease, and organised Dr Jorg L. Neumann to provide a translation from Germar; Dr Patricia Price, Wound Healing Research Unit, University of Wales, Cardiff, Dr Henri Stoffers, Department of General Practice, University of Maastricht and Professor Vincent Falanga, Department of Dermatology, Boston University; all graciously provided imprints of articles, letters and books at their own cost that were essential to parts of this review. The Effie Redmond Trust, the Stella Luty Trust and the New Zealand Centre for Evidence Based Nursing provided assistance with the funding of this project. Auckland Healthcare Services have assisted my continued education throughout my Master's degree. John McArthur, Director of the New Zealand Centre for Evidence Based Nursing, provided me with not infrequent opportunities to vent my frustrations with thesis projects. Professor Alison Dixon, Department of Nursing and Midwifery, Victoria University of Wellington, provided gentle guidance and entrusted me with a scholastic freedom that I have endeavoured to respond to. Finally, neither this thesis nor my Master's degree would have been possible without the love, release from so many domestic and parental responsibilities, and support of my partner, Kay Suzanne Parish. 


\section{For Kay,}

who foresaw how difficult this year would be and just how occasionally our hearts might go swimrning,

and

Rhys \& Daphne,

our two precious children,

whiose desire to learn humbles my own. 


\section{TABLE OF CONTENTS}

$\begin{array}{lll}\text { Abstract } & \text { ii }\end{array}$

Acknowledgements

List of Tables vii

List of Figures vii

Chapter 1: Introduction 1

Epidemiology of Leg Ulceration $\quad 1$

Causation of Venous Leg Ulcers $\quad 4$

Socio-economic Impact of Venous Ulceration 9

Treatment of People with Venous Ulceration $\quad 14$

Pentoxifylline $\quad 14$

$\begin{array}{lr}\text { Chapter 2: Methodology } & 16\end{array}$

Development of Research Synthesis in the Social Sciences 18

Development of Research Synthesis in Health Sciences 21

Conducting a Meta-analysis $\quad 25$

Conclusion $\quad 33$

$\begin{array}{ll}\text { Chaptex 3: Method } & 34\end{array}$

Search Strategy $\quad 34$

Criteria for Inclusion of Studies $\quad 35$

Initial Selection of Studies $\quad 36$

Translation of Studies $\quad 36$

Selection of Included Studies $\quad 36$

Data Extraction $\quad 37$

Data Synthesis $\quad 37$

Chapter 4: Data Collection and Synthesis 41

Details of Excluded Studies $\quad 41$

Description of Included Studies $\quad 43$

Methodological Quality of Included Studies $\quad 46$

Comparisons of Studies $\quad 48$

$\begin{array}{ll}\text { Chapter 5: Discussion } & 53\end{array}$

Implications for Clinical Practice $\quad 58$

Implications for Research $\quad 60$

Chapter 6: Criticism and Non-Random Reflections 63

Personal Reflections on Meta-analysis $\quad 64$

Nursing and Meta-analysis $\quad 66$ 
Chapter 7: Conclusions

Epilogue: Personal Reflections on Nursing Research

Appendix 1: Contact Addresses of Co-reviewer

Appendix 2: Search Strategy for Cochrane Wound

Review Group

Appendix 3: Search Strategy for Cochrane Peripheral Vascular

Diseases Group

Appendix 4: Inclusion/Exclusion Coding Sheet

Appendix 5: Data Extraction Coding Sheet

95

Appendix 6: Evidence Tables

97

References

102 


\section{LIST OF TABLES}

Table 1 Prevalence of chronic venous ulceration related to age and sex

Table 2 Causes of leg ulcers

Table 3 Diagnostic assessments utilised to determine venous aetiology in included studies

Table 4 Characteristics of populations of included studies - Mean age and sex ratio

Table 5 Characteristics of populations of included studies - Mean ulcer size and duration

\section{LIST OF FIGURES}

Figure 1 Cycle of causative mechanism of venous ulceration

Figure 2 Cycle of causative mechanism of venous ulceration

Figure 3 Treatments with factorial trial of Dale et al (1999)

Figure 4 Comparison of pentoxifylline and compression versus placebo and compression (fixed effects model)

Figure 5 Sensitivity analysis - excluding $2400 \mathrm{mg}$ arm (fixed effects model)

Figure 6 Sensitivity analysis - double blind studies only (fixed effects model)

Figure $7 \quad$ Sensitivity analysis - including participants excluded after (fixed effects model)

Figure 8 Sensitivity analysis - excluding trials not specifying an outpatient only setting (fixed effects model).

Figure 9 Sensitivity analysis - excluding trials not specifying oral treatment only (fixed effects model)

Figure 10 Sensitivity analysis - exclusion of trials not specifying participants' sex or counter to trends in prevalence studies (fixed effects model)

Figure 11 Sensitivity analysis - diabetic participants (fixed effects model)

Figure 12 Trial duration 8-12 weeks (fixed effects model)

Figure 13 Trial duration 24 weeks (fixed effects model)

Figure 14 Sensitivity analysis - Trial duration 24 weeks excluding $2400 \mathrm{mg}$ arm (fixed effects model)

Figure 15 Adverse effects, pentoxifylline versus placebo (fixed effects model)

Figure 16 Funnel plot 


\section{Chapter 1. INTRODUCTION}

Approximately 1 percent of the population are thought to have leg ulcers at some point during their lives (Dale, Callam, Ruckley, Harper, \& Berrey, 1983). Widmer (1978) found 1.3 percent of 4376 adult workers in the Basle chemical industry had suffered or were currently suffering from leg ulcers. Leg ulcers are defined as a defect in the skin, below the level of the knee, persistent for 4 weeks or longer (Baker, Stacey, Jopp-McKay, Hoskin \& Thompson 1991), although Nelzén, Berqvist, and Lindhargen (1991) use 6 weeks as the threshold of chronicity. These definitions may also include isolated foot ulcers. Ulcers of venous aetiology are also known as varicose ulcers, stasis ulcers and gravitational ulcers. This chapter explores the prevalence of leg ulceration and venous leg ulcers, the causative mechanism of venous ulceration, the socioeconomic impact of leg ulceration and treatment options for people with venous ulcers.

\section{Epidemiology of Leg Ulceration}

Callam, Ruckley, Harper, and Dale (1985) investigated active leg ulceration in two Scottish Health Board areas encompassing a population of one million people. Surveys were posted to all general practitioners, district nurses, occupational health nurses, outpatient clinics, acute and long-stay hospital wards, old people's homes and physiotherapy departments, with returns cross-checked to ensure duplicate reports were not counted. A total of 1477 people were identified as having leg ulcers, giving a point prevalence for active ulcers of $1.48 / 1000$, with prevalence increasing with the age of the population. Lees and Lambert (1992) also used a postal survey, distributed to district nurses with a caseload of patients with ulcers within an urban area encompassing a population of approximately 240,000. Eighty-four percent of nurses replied, reporting 206 patients aged 45 or more, currently being treated for ulceration. This converted to a prevalence of 1.9/1000; the authors also reported the incidence as $3.5 / 1000{ }^{1}$ In relying upon patients being known to health workers (thus failing to estimate those self-caring) and focusing only on active ulceration (thereby excluding those people who were 'between' ulcers), these studies are likely to have underestimated the true prevalence of leg ulceration.

\footnotetext{
${ }^{1}$ Prevalence is the percentage of a population with target disorder; incidence is the proportion of new cases in the population with the target disorder (Sackett, Haynes, Guyatt, and Tugwell, 1991).
} 
Venous disease, in the form of superficial or deep venous insufficiency, is thought to be causative of the majority of leg ulcers. Early reports were descriptive, relying on clinical history and examination to determine aetiology. Anning (1954) reported a case series of 1026 patients with ulcers, identifying venous disease as causative in all. Cornwall, Dore and Lewis (1986) randomly selected 150 people from a cross-section study investigating the prevalence of leg ulcers. Sixty-seven percent accepted the invitation to have an assessment and non-invasive physical investigation of the aetiology of their leg ulcer. ${ }^{2}$ They found thirty-one percent had ischaemia, and ten percent had other aetologies, leaving the reader to intuit that fifty-nine percent of the sample had a venous causation of their leg ulcer. Callam, Harper, Dale, and Ruckley (1987), using a similar approach, investigated the aetiological distribution of 600 participants from a previous sampling frame of 1477 people with open leg ulcers (Callam, et al., 1985). They found 76 percent had evidence of venous disease, 22 percent had arterial insufficiency, and 5 percent diabetes.

Only two published studies have systematically investigated the aetiology of leg ulcers in a cross-section of defined populations. Baker, et al. (1991) sampled the population of Perth $^{3}$ over a three-month period, drawing participants from self-referral, institutions, and health professionals. All participants provided a clinical history, and underwent a physical assessment including a non-invasive vascular examination by photoplethysmography. The investigators found 259 people with a chronic ulcer ${ }^{4}$, ninety-three percent (242 participants) of whom consented to be assessed. An abnormal refilling time was found in 138 participants (with 163 ulcerated legs), representing fifty-seven percent of the sample. ${ }^{5}$ The investigators assumed the 242 participants to be representative of all those identified with ulcers, and calculated the prevalence of chronic venous ulceration as $0.62 / 1000$, with prevalence increasing with age (Table 1). It should be noted that this included a very small number of foot ulcers, determined to be of venous origin.

\footnotetext{
2 Using photoplethysmography and Doppler ultrasound. Photoplethysmography examines the competence of valves in the venous bed, while Doppler ultrasound is used to determine the AnkleBrachial Pressure Index.

${ }^{3}$ Estimated population 238,000 in June 1988.

${ }^{4}$ An ulcer was defined as a defect in the dermis below the knee, including foot ulcers, lasting for 4 or more weeks.

5 A refill time less than twenty seconds, as determined by photoplethysmography is considered indicative of venous insufficiency (Porter, et al., 1988), although Baker, et al. have used a more conservative twenty-five seconds as the threshold.
} 
Table 1: Prevalence of chronic venous ulceration related to age and sex; adapted from Baker, et al. (1991)

\begin{tabular}{lcccc}
\hline & \multicolumn{2}{c}{ Male } & \multicolumn{2}{c}{ Female } \\
Age & Number & Prevalence & Number & Prevalence \\
\hline $0-39$ & 1 & 0.015 & 2 & 0.029 \\
$40-49$ & 0 & 0.0 & 2 & 0.15 \\
$50-59$ & 4 & 0.38 & 5 & 0.46 \\
$60-69$ & 13 & 1.5 & 13 & 1.3 \\
$70-79$ & 16 & 3.3 & 29 & 4.0 \\
$80+$ & 15 & 8.3 & 38 & 9.1 \\
\hline
\end{tabular}

Nelzén, et al. (1991) aimed to identify all people with current leg ulcers, including foot ulcers, known to health professionals within a defined population of 270,800 in Sweden. Eight hundred and twenty-seven people were identified, and half of this group was randomly sampled to attend a clinical examination, consisting of a history and physical assessment with Doppler ultrasonography, to determine ulcer aetiology. Ninety-two percent of the sample accepted the invitation to participate and this group corresponded with the original sample on age and sex distribution. All participants were examined by the same clinician and the aetiology was recorded as one of eleven categories (Table 2, see page 4). Fifty-four percent of the 382 participants were determined to have ulcers of primarily venous aetiology ${ }^{6}$, giving a point prevalence of 1.6/1000 (95\% confidence interval 1.5-1.8/1000). Ninety-five percent of the ulcers were on the gaiter area of the leg; excluding the isolated foot ulcers increased the percentage of venous leg ulcers from fifty-four percent to seventy percent. As with Baker, et al. (1991), prevalence of ulceration increased with age.

Nelzén, et al. (1991) explain the greater prevalence in their study as being a factor of population difference; the percentage of the population older than 65 was 18.4 percent in the Swedish study compared to 12.4 percent in Baker, et al. (1991). However, both Baker, et al. (1991) and Nelzén, et al. (1991) believed they have under-reported the true prevalence of ulceration. Neither study attempted to estimate the numbers of people 'between' ulcers, and whilst Baker, et al. (1991) attempted to accrue people who might be self-caring of their ulcers through a newspaper article at

${ }^{6}$ Ulcers in legs with ultrasonic proof of reflux in the venous bed and absence of any other major aetiological factor. 
the start of the study period, this was not followed up over the course of the study. Nelzén, et al. (1991) only approached health professionals to identify those with an open ulcer and did not attempt to determine

Table 2: Causes of leg ulcers (percentages rounded); adapted from Nelzén, et al. (1991)

\begin{tabular}{lcc}
\hline \multicolumn{1}{c}{ Aetologic Group } & All Ulcers & Ulcers above the foot \\
\hline Venous & $184(40)$ & $182(52)$ \\
Mixed venous/arterial & $66(14)$ & $65(18)$ \\
Mixed arterial/venous & $20(5)$ & $15(4)$ \\
Arterial & $34(7)$ & $13(4)$ \\
Arterial/diabetes & $43(9)$ & $5(2)$ \\
Diabetes & $18(4)$ & $2(1)$ \\
Traumatic & $9(2)$ & $8(2)$ \\
Pressure & $15(3)$ & $1(<1)$ \\
Multi-factorial & $48(11)$ & $40(11)$ \\
Other single causes & $26(6)$ & $22(6)$ \\
\hline
\end{tabular}

numbers of people who might be self-caring. Thus, the true extent of the problem is still concealed, hidden by the methodological approaches of recent attempts to quantify the prevalence of leg ulceration, the desire of some people to care for their own ulcer, and possibly by the variety of health professionals who care for patients with leg ulcers ${ }^{7}$ (Ruckley, 1997).

\section{Causation of Venous Leg Ulcers}

Hippocrates is said to have recognised the relationship between ulceration and superficial varicosities 2000 years ago, as did the Roman physicians Aurelius Cornelius Celsus, Claudius Galen and Aetios of Amida (Negus, 1995). With the rise of the humoral theory in the Dark Ages, ulcers were thought to be caused by the efflux of dangerous humours, but this theory began to lose ground from the seventeenth century, as the understanding of thrombus formation began to be understood. ${ }^{8}$ John Gay introduced the term 'venous ulcer' and described the role of

7 Plastic surgeons, vascular surgeons, dermatologists, rheumatologists, geriatricians, general practitioners, nurse specialists and district nurses.

${ }^{8}$ Rudolph Virchow first used the term fibrinogen and described his triad of the causes of thrombi in 1860. 
damage to the deep veins by thrombus formation in 1868 (Browse \& Burnand, 1982; Negus, 1995). This theory found few adherents, but was revived by John Homans in 1917, who noted that few venous ulcers occur in the presence of varicose veins and described the destruction of venous valves by thrombus formation ${ }^{9}$ (Negus, 1995). Thrombosis destroys the deep veins, leaving either, narrow irregular and valveless channels if the vein recanalises, or tortuous dilated collateral veins with incompetent valves if the deep vein does not recanalise (Browse, 1983).

When the legs are in a dependent position, a significant volume of blood is held in the venous bed of the leg, $100-140 \mathrm{ml}$ in the sinusoids alone. This is approximately equivalent to that held in the heart (Negus, 1995). Quietly standing, without any movement of the legs, the muscles of the lower extremity rhythmically contract and relax, ejecting blood from the superficial venous system into the deep veins, the sinusoids of the gastrocinemius and soleus muscles, and then into the proximal veins, aiding venous return to the heart. It is thought that this pumping action, although not required to maintain venous return to the heart, "is of vital importance to preserve the integrity of the microcirculation, by reducing distal capillary pressure when standing" (Stranden \& Kroese, 1995, p59). The venous pumps are located in the thigh (quadriceps, sartorius and hamstring pumps), and most importantly, in the calf muscles. During the systole of the calf pump, competent valves in the deep and communicating veins protect the superficial venous system from the high contractile pressures of up to $250 \mathrm{mmHg}^{10}$ within the muscle. In the absence of any pathology, this is seen in ambulatory venous hypotension during exercise, where the pressure in the dorsal vein of the foot falls from $90 \mathrm{mmHg}$ to $20 \mathrm{mmHg}$ (Negus, 1995). The valves also prevent backflow during the diastole, and the veins are refilled. Without the venous pumps acting as a compensatory mechanism, severe capillary distension would occur from exposure to high pressures during the systole of the calf pump (ibid.). Obstruction of the deep veins by thrombi, or destruction of the valves in the communicating or deep venous systems may reverse the blood flow from superficial to deep to proximal veins on exercise, leaving the superficial veins distended by high pressure throughout exercise by ambulatory venous hypertension.

Despite the knowledge that venous ulcers occur in the presence of calf muscle pump

9 Varicose veins in the absence of any deep venous damage are termed superficial venous insufficiency. 
deficiencies, the mechanism by which the ulceration occurs is unknown. The two principle explanations of the early to mid-twentieth century, venous stasis and arterio-venous shunting, have been rejected. Browse and Burnand (1982) noted that venous stasis, or stagnant blood lying close to the skin causing tissue anoxia was debunked by the finding that oxygen saturation in the blood of venous ulcers was higher than in the non-affected limb of participants. The higher oxygen content and faster blood flow observed in venous ulcers was then considered to be the result of shunting through microscopic fistulas between arterioles and venules, resulting in anoxia of the overlying tissues. However, Browse and Burnand (1982) observed that examination of people's ulcers with radioactively labelled macroaggregates had failed to show any such fistulas, and therefore proposed a new hypothesis (Browse \& Burnand, 1982; Browse, 1983). They had noted that skin biopsies of patients with deep venous insufficiency showed fibrin cuffs around the capillaries. Browse and Burnand (1982) suggested that the venous hypertension increased the intraluminal pressure of capillaries, stretching the interendothelial pores, and allowing large molecules such as fibrinogen to escape into the interstitium at greater than normal rates. This was converted into fibrin, but the abnormal volumes exhausted the fibrinolytic activator, allowing the creation of pericapillary fibrin cuffs which prevented the diffusion of oxygen, leading to tissue anoxia, cell death and ulceration (Figure 1, see page 7).

Other investigators have proposed a theory that incorporates fibrin cuff formation, but advance that this is not the causative mechanism of ulceration, only a by-product of the aggregation of white blood cells. Coleridge Smith, Thomas, Scurr and Dormandy (1988) noted that there is no evidence that a fibrin cuff is a barrier to diffusion, and that other investigators had found areas of skin under capillary fluorescence microscopy with no apparent blood flow. They hypothesized that the slowing of venous perfusion by hypertension caused the capillaries to become plugged by white cells. The trapped white cells release proteolytic enzymes and superoxide radicals resulting in endothelial damage. This causes the increased permeability with the consequent loss of fibrinogen and formation of a fibrin cuff. However, Coleridge Smith, et al. (1988) hypothesize it is the trapped white cells that prevent circulation in the affected capillaries with the subsequent anoxia resulting in tissue death in the area surrounding the capillaries (Figure 2, see page 8). Mirshahi,

${ }^{10} \mathrm{mmHg}$ represents pressure measured in millimetres of mercury. 


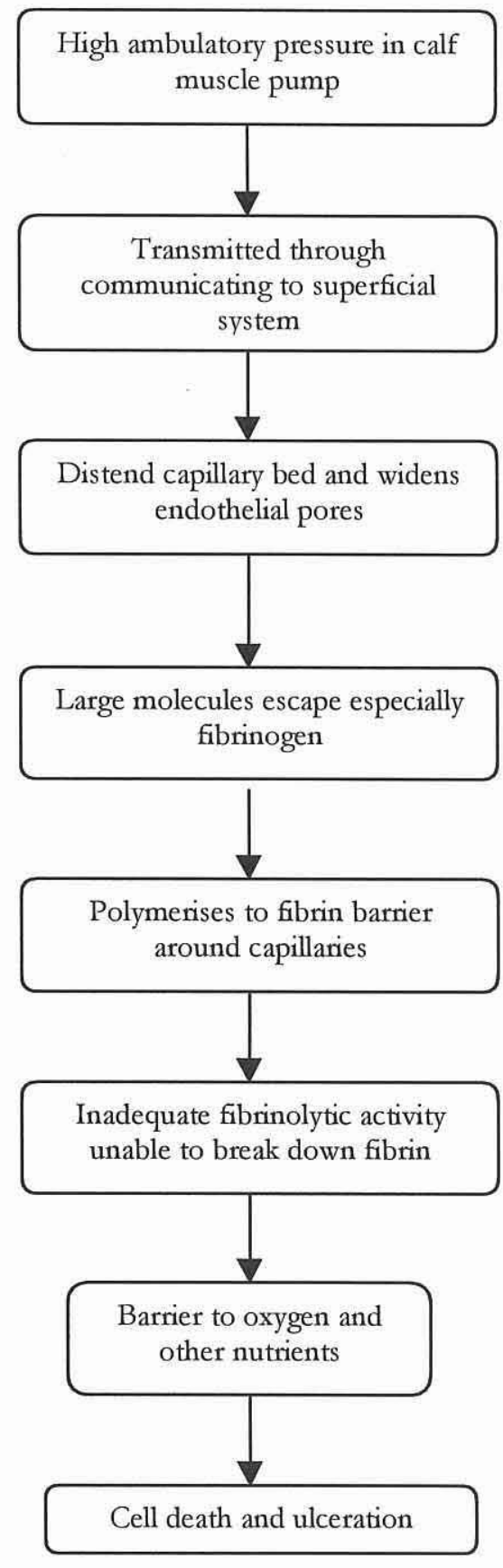

Figure 1: Cycle of causative mechanism of venous ulceration; adapted from Browse and Burnand (1982).

Soria, Mirshahi, Soria, Lenoble, Vasmant, Cambazard and Claudy (1995), in a double blind randomised controlled trial of 10 people with venous ulcers, found lower levels of fibrin and elastase (an enzyme released by activated white cells) in the group treated with pentoxifylline. Pentoxifylline is known to reduce white cell 
aggregation and fibrinogen loss (Stellin \& Waxman, 1989). The authors concluded this evidence was supportive of the white cell trapping theory, and suggested that "inflammation generated by activated white blood cells that accumulate with unrelieved venous pressure is the first event in chronic leg ulcer" (ibid. pS105).

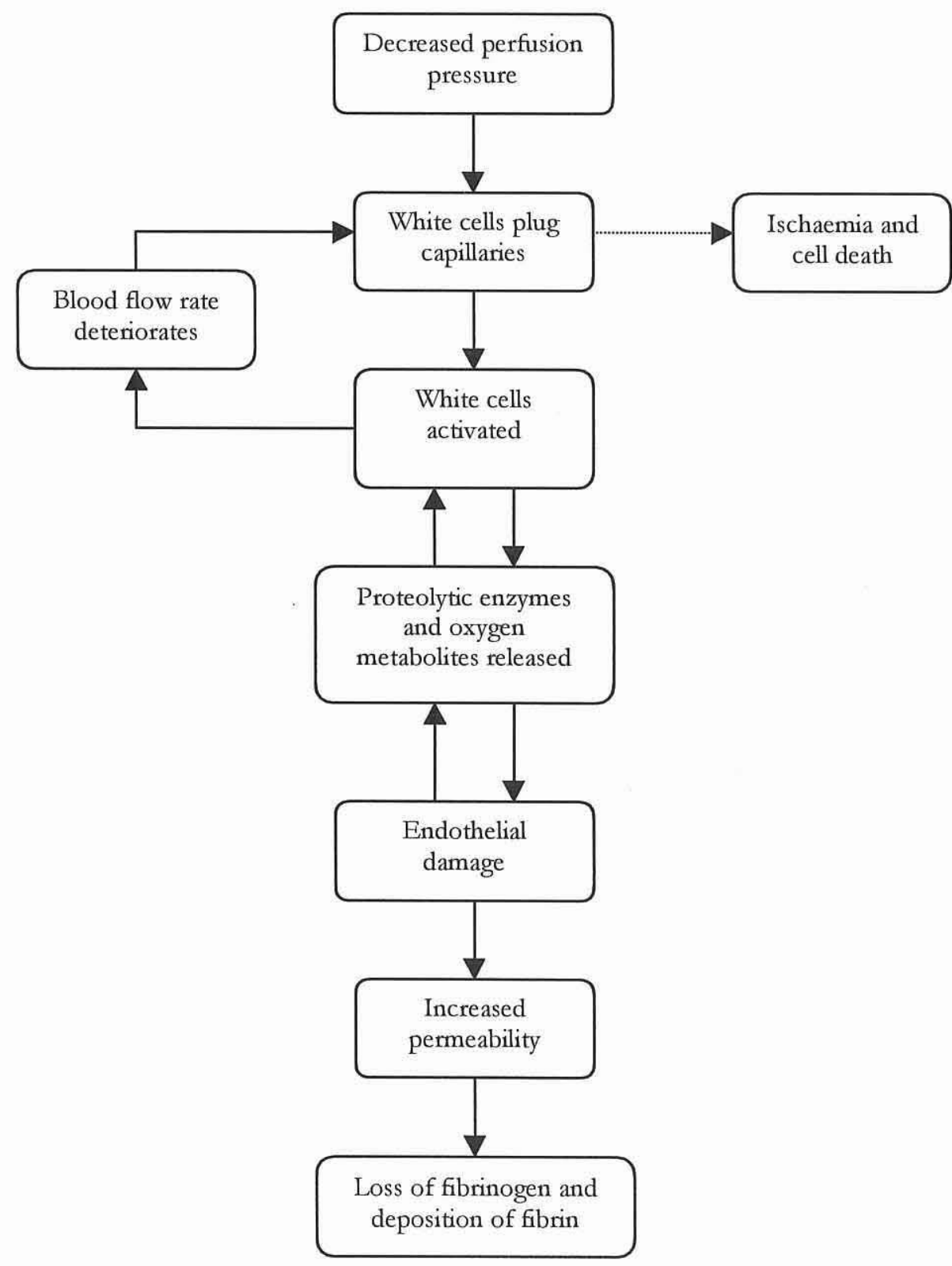

Figure 2: Cycle of causative mechanism of venous ulceration; adapted from Coleridge Smith, et al. (1988). 


\section{Socio-economic Impact of Venous Ulceration}

The impact of ulceration on perceived health is considered in terms of quality of life. There are few studies available in English that have examined this aspect of leg ulceration, fewer still that have examined the problems confronted by people with venous ulceration (Lamping, 1997; Ruckley, 1997). However, Lamping (1997) notes that quality of life studies are assuming more importance because the traditional definition of health has broadened to incorporate physical, mental and social wellbeing, health interventions are recognised to have an effect on other outcomes than morbidity and mortality, and purchasers are demanding increased evidence of costeffectiveness in terms of health outcomes meaningful to patients. Quality of life (QoL) has been studied using well-validated generic tools, as well as qualitative studies. The Short Form Health Survey-36 (SF 36) has been used in one study (Price \& Harding, 1995; Price \& Harding 1996), whilst the Nottingham Health Profile (NHP) has been used in three studies, two in Britain and one in Sweden (Lindholm, Bjellerup, Christensen, \& Zederfeldt, 1993; Hamer, Cullum, \& Roe, 1994; Franks, Bosanquet, Brown, Straub, Harper, \& Ruckley, 1999). The richer texts of the descriptive and the qualitative approaches provide the existential narratives of people living with chronic ulceration, which despite non-probability sampling techniques, are consistent with the data of the quantitative approaches (Phillips, Stanton, Provan, \& Lew, 1994; Charles, 1995; Walshe, 1995; Chase, Melloni, \& Savage, 1997).

Price and Harding (1996) used the SF-36 to assess 55 patients, 37 women and 18 men, against age-equivalent norms on eight sub-scales. ${ }^{11}$ The patient group rated themselves more poorly on all sub-scales except for mental health "confirming clinical impressions that these patients have a condition that affects the QoL" (ibid., p445). The male participants rated themselves as significantly healthier than the women on physical functioning $(p=0.006)$, vitality $(p=0.028)$ and social functioning $(p=0.017)$. The authors also note that when followed up 4 months later, the self-ratings had not changed significantly despite the ulcers having healed in 35 percent of the patients. Price and Harding suggest that this may be a limitation of the generic measures of health-related quality of life (HRQOL), and that disease-specific tools should be developed for people with chronic wounds. Studies using the NHP also found that

11 The 8 subscales of the SF-36 are physical functioning, social functioning, role limitation (physical), role limitation (emotional), pain, vitality, general health perception and mental health. 
people with chronic ulcers are burdened by their ulcer. Lindholm, et al. (1993) administered only part 1 of the two part NHP questionnaire ${ }^{12}$ to 154 consecutive patients with venous, mixed aetiology and arterial ulcers attending a Department of Dermatology clinic over 13 months. One hundred and twenty-five questionnaires were evaluated. The mean global score was 173 percent of normal (age and sexadjusted), indicating significantly lower perceptions of well-being. These scores varied considerably between men and women, with men scoring lower on perceived health (232 percent of normal) than women (132 percent of normal), thus contradicting the findings of Price and Harding (1996). ${ }^{13}$ Lindholm, et al. (1993) found men scored higher than women on every parameter, and globally men and women scored higher than normal on each parameter. The exception was that the women with ulcers scored normal on social isolation.

Franks and Moffatt (1998) noted that the gender difference observed by Price and Harding (1996) was reversed in the study by Lindholm, et al. (1993), and hypothesized that this was a consequence of Price and Harding (1996) using raw scores, whereas Lindholm, et al. used expected age/sex adjusted values of the normal population. ${ }^{14}$ Franks and Moffatt (1998) tested 758 people with leg ulcers that had been identified by a previous prevalence survey using the NHP. They found the participants had significantly higher NHP scores on all six scales, indicating poorer perceived health-related quality of life than age/sex matched normal values. Increasing age lead to greater deficits in energy, mobility and social isolation. Using raw scores, women had lower perceived HRQOL than men, but this would be expected as $64 \%$ of the sample were women and the sample became more skewed towards women as the age of the participants increased. When scores were adjusted for age/sex matched normal values, it was the younger people that had the greatest deficit in perceived HRQOL in all domains and men had lower perceived HRQOL than women in the domains of bodily pain, sleep and social isolation and energy.

\footnotetext{
12 The 6 areas rated in the Nottingham Health Profile are pain, physical mobility, sleep, energy, emotional reactions and social isolation.

${ }^{13}$ It should be noted that Price and Harding (1996) considered their study to be exploratory, rather than definitive.

${ }^{14}$ Franks and Moffatt (1998) also suggested the difference may be as a consequence of fundamental differences between the two populations and that the studies used different tools that have subtle variations in scoring. However, the authors believed the difference most likely derived from the use of controls or age and sex matched normal values.
} 
A descriptive quantitative study using standardised interviews reinforces the findings of the HRQoL studies using generic questionnaires. Phillips, et al. (1994) interviewed 62 people ( 25 men and 37 women) about the financial, psychological, functional and emotional impact of leg ulceration. They found that 81 percent believed their mobility was adversely affected, with men reporting more immobility than women; that in 42 percent of those not working, ulceration was a factor in the decision to stop working, and that in all participants still working, their work capacity was limited by ulceration. Sixty-five percent of participants reported severe pain associated with their ulcer and 68 percent said the ulcer had a negative emotional impact, with the variety of emotions being fear, social isolation, anger, depression and negative selfimage. This was significant in younger people "who tended to be more depressed about their condition and have a more negative self-image" (Phillips, et al., 1994, p52). Despite the fact that 71 percent had other severe medical problems, such as hypertension and cardiac disease, all participants believed that their leg ulcer was their most significant problem. Multivariate analysis revealed that the primary concerns of the participants were the ability to walk, accomplish everyday tasks and maintain mobility.

The above studies included participants with ulcers of any type in their samples. Three phenomenological studies focused on the experiences of people with venous leg ulceration, with remarkably consistent results (Charles, 1995; Chase, et al., 1997; Walshe, 1995). Chase, et al. (1997) identified 4 major themes from interviews with 7 patients - 'a forever healing process', 'limits and accommodations', 'powerlessness' and 'who cares?' A 'forever healing process' related to patients having had extensive histories of ulceration, with half not able to remember their first episode. Healing no longer had the positive connotations of overcoming an acute illness; healing comes to mean a constant attention to the positive and negative changes in the body, and a constant need for health care. It is not an isolated episode of professional care and attention, "it's like a forever bealing process, not getting worse, not getting better" (Chase, et al., 1997, p74). The second theme identified by Chase, et al. was limits and accommodations'. Participants described major limitations in activity as a consequence of pain and mobility impairments: "I cannot do anything I want. I cannot go any place ... I kind of got used to it" (ibid. p75); "People can't believe the pain you bave with an ulcer" (Charles, 1995, p574). Lifestyle limitations were also a result of job loss, treatments and self-imposed restrictions. Negative body images associated with open wounds meant that many participants reported the need to hide their bodies, with 
clinics being the only place participants were told their legs looked good. Both Charles (1995) and Walshe (1995) report the restrictions caused by immobility and participants' need to disguise or hide their ulceration: "I couldn't stand and do things like bousework. I used to be a competitive swimmer before I had the ulcer. I didn't swim any more, not even with my children" (Charles, 1995, p574). This theme has significant impact on human interaction, with social isolation increasing, especially as participants drop out of the workforce, or age.

Chase, et al. (1997) described the third theme as 'powerlessness', revealed in the effects of slow wound healing and the likelihood of recurrence, and patients not being able to control the healing outcome. One participant described his ulcer as running his life: "I have to take more care of my foot than I bave to take care of myself" (Chase, et al., 1997, p76). Participants quoted by Walshe (1995) described this as the uncertainty of healing, reinforced by the lack of a known timescale, whereas Charles described this as hopelessness, helplessness and lack of control: "It broke down every year. Every year at the same spot. It gradually got bigger and then stayed the same size" (Charles, 1995, p574). Even patients, who had an internal locus of control, usually associated with an active approach to problem solving, were worn down by the 'forever healing' and came to believe they had no control over ulceration (ibid.). The fourth theme, 'who cares?' describes both who treats and manages the participant's ulcer Some participants managed their own ulcers, although neither Charles (1995) nor Walshe (1995) report this. Walshe does note that participants tended to hand over control of their treatments to professionals and rarely were included in the care. Charles expands this theme to incorporate concerns about whether health workers actually care about their patients or know what they are doing:

In hospital, at first the sister took me into the dressing room and pointed to several applications She stopped at [product A], and for three years I was stuck with [product A]. You are just a number, another tin of baked beans, they walk you in, dress you and walk you out.

(Charles, 1995, p574)

The economic impact of venous leg ulceration is considered significant, but has mainly been reported as a cost to the health system. Only one study has considered the economic impact on those afflicted with leg ulceration, venous or otherwise. 
Phillips, et al. (1994) reported both the impact of ulceration on work capacity as well as patient estimates of annual costs they bear for ulcer treatment. Leg ulceration was significantly correlated with time lost from work and job loss. Expenses incurred included costs of medical care, time lost from work, cost of dressing and transportation costs. ${ }^{15}$ More than 30 percent of the participants reported costs of between $\$ 101$ and $\$ 1000$ per annum, with time lost from work being the smallest element of this. However, time lost from work was estimated at more $\$ 1000$ per annum by 25 percent of participants.

The cost to the health service of caring for people with venous ulceration in Britain has been estimated at between $£ 230-£ 400$ million at 1991 prices (Bosanquet, 1992). Laing has estimated that people with venous diseases utilised 1.5-2 percent of the total health care budgets in Britain, France and West Germany at 1992 exchange rates (in Ruckley, 1997). Harkiss (1985) estimated the 4 month costs for a variety of products used by community nurses to dress leg ulcers, as ranging from $£ 132$ to £1290. This figure was dependent on the type of product selected as well as the number of dressing changes per week. It did not include indirect costs such as nursing time or vehicle costs. More precise estimated costs are reported from randomised controlled trials. Franks, Bosanquet, Brown, Straub, Harper, and Ruckley (1999) found the total direct and indirect costs of treating 200 patients over 24 weeks to be approximately $£ 193,000$, with a cost per healed limb of $£ 1654$. The 41 percent of people whose ulcers remained unhealed after the trial concluded continued to represent a significant cost to the health service. Morrell, Walters, Dixon, Collins, Brereton, Peters and Brooker (1998), in a cost-effectiveness study of specialist ulcer service which achieved better healing rates than district nursing, estimated the direct and indirect costs of the ulcer service at $£ 878$ per patient per year compared to the 'normal' treatment group $£ 859 .{ }^{16}$ Again the 44 percent of participants whose ulcers remained unhealed at the completion of the trial remained a cost to the health system.

\footnotetext{
15 This study was conducted in the United States and estimated costs will reflect the American model of health care.

16 The superior healing rates achieved by the specialist service compensated for the minor cost difference making the specialist ulcer service the more cost-effective service.
} 


\section{Treatment of People with Venous Ulceration}

The therapeutic mainstay in management of venous ulceration is graduated compression bandaging, achieving pressures of $23 \mathrm{mmHg}$ at the ankle and decreasing as it ascends to the knee. A systematic review found high compression bandaging of any nature (3 layer, 4 layer or short stretch bandaging) achieved healing in larger proportions of people with venous ulcers than low compression bandaging or no compression (Cullum, Fletcher, Nelson, \& Sheldon, 1999). Despite this many people's ulcers remain unhealed after 3 months of compression therapy, with the proportions of people with unhealed ulcers varying ranging from 25 to 60 percent (Hendricks \& Swallow, 1985; Kitka, Schuler, Meyer, Durham, Eldrup-Jorgensen, Schwarcz \& Flanigan, 1988; Charles, 1991; Duby, Hofman, Cameron, DobloffBrown, Cherry \& Ryan, 1993; Wilkinson, Butterfield, Cooper, \& Young, 1997). Adjunctive treatments need to be considered for people with ulcers refractory to compression therapy. Such treatments include extended bedrest, split skin grafting, pinch grafting, hyperbaric oxygen therapy, as well as pharmacological treatments. One promising pharmacological agent is pentoxifylline.

\section{Pentoxifylline}

Pentoxifylline was developed by Albert-Roussel, a subsidiary of Hoechst AG. It is a xanthine derivative that has haemorrheolgical properties and first became available commercially in 1972, primarily for the treatment of intermittent claudication. Haemorrheology is a term coined in 1952 by Alfred Copley and is concerned with the flow properties of blood (Savitsky, 1986). Fluid mechanics is influenced by the interrelationship between the length and diameter of the tube through which a fluid is moving, the pressure within the tube and the viscosity of the fluid. Normally blood viscosity is low, but this can be increased by a greater percentage of erythrocytes in the plasma, a decrease in the rate of flow produced by an obstruction or the presence of abnormal levels of proteins in the plasma (Savitsky, 1986).

Erythrocytes are approximately 8 microns in size and disc shaped when moving through major vessels, but must flex, bend or elongate to move through capillaries, which have a diameter of between three and five microns. Tissue ischaemia creates an anaerobic cascade, releasing lactic acid and causing local acidosis and 
hyperosmolarity. This seems to cause erthyrocytes to lose flexibility by decreasing intracellular adenosine triphosphate (ATP) and enhancing local hypoxia (Stellin \& Waxman, 1989). Pentoxifylline has been shown to improve erthyrocyte flexibility by inhibiting erthyrocyte phosphodiesterase, thus increasing available ATP (Spittell, 1986). More importantly with regard to microcirculatory causes of venous ulceration, pentoxifylline has been shown to influence leukocyte migration, decrease fibrinogen concentrations, and inhibit platelet aggregation. In low flow states, leukocytes are activated and adhere to capillary walls, releasing oxygen metabolites and proteolytic enzymes (Stellin \& Waxman, 1989). Leukocytes, though smaller in number than erythrocytes (approximately 1:700), can significantly contribute to plasma viscosity (Ward \& Clissold, 1987). Pentoxifylline destabilises polymorphonulcear leukocyte membranes, exposing the cell to chemotactic agents that encourage cell migration (Stellin \& Waxman, 1989). Pentoxifylline also decreases plasma fibrinogen levels either through increasing fibrinolytic activity or decreasing the production of fibrinogen (Ward \& Clissold, 1987; Stellin \& Waxman, 1989). Mirshahi, et al. (1995) demonstrated that the expression of the enzyme elastase and creation of fibrin was reduced in people with leg ulcers, following treatment with pentoxifylline. This may be a result of the drug's action on leukocytes and fibrinogen. Pentoxifylline also inhibits the formation of thromboxane, a major stimulant to the aggregation of platelets, probably by inducing the synthesis and release of the precursor, prostacyclin (Spittell, 1986; Ward \& Clissold, 1987).

The therapeutic actions described above prompted investigators to research the effectiveness of pentoxifylline in the treatment of venous ulceration, as an adjunct to compression therapy. These studies, tending to small numbers, different outcome measures and methodological problems, have produced confusing results. Two of the larger studies produced a significant result in favour of pentoxifylline, and an insignificant result, in the direction of pentoxifylline (Colgan, Dormandy, Jones, Schraibman, Shanik, \& Young, 1990; Dale, Ruckley, Harper, Gibson, Nelson, \& Prescott, 1999). That conventional treatment using compression bandaging does not heal all people with venous leg ulcers means that other treatment modalities deserve investigation. Any adjunctive treatment that improves rates of healing may hold promise for those that do not respond to compression alone. Therefore, it is the aim of this investigation to use meta-analysis in order to determine whether pentoxifylline is effective at improving rates of healing for patients with venous leg ulcers. 


\section{Chapter 2. METHODOLOGY}

Review articles are a necessary disciplinary tool "because they deliberately aim at cumulating knowledge in an area" (Feldman, 1971, p86). The actual purpose may vary from review to review. Reviews may attempt to explore the impact of developments in a field, verify existing theories or develop new ones, synthesize knowledge from different lines, or infer generalisations about substantive hypotheses from a set of studies directly bearing on those hypotheses (Jackson, 1980). The subject of this chapter is reviews that meet Jackson's fourth purposes; those that attempt to draw generalisations from different studies that have tested a similar hypothesis. Such reviews are known by a variety of names: data pooling, pooled analyses, integrative reviews, meta-analyses, met-analyses, quantitative review, research integration, research syntheses, research overviews, systematic reviews and systematic overviews (Taveggia, 1974; Glass, 1976; Glass, 1977; Jackson, 1980; Gerbag \& Horwitz, 1988; Jenick, 1989; Dickersin \& Berlin, 1992; Cooper \& Hedges, 1994; Cook, Sackett, \& Spitzer, 1995; Finney, 1995). Glass (1976) distinguishes such reviews from secondary analyses, which are the re-analysis of data for the purpose of re-evaluating data with better statistical techniques in order to better answer the original research question. Research synthesis is used as a generic term throughout this chapter and refers to the retrieval and analysis of results from individual studies for the purpose of integrating the studies' findings. Research synthesis can thus include both qualitative and quantitative methods of synthesis.

It is common to all literature reviews that they are not based on new facts, but that they utilise published material as a base and synthesize the information from previous publications in some manner (Cooper \& Hedges, 1994). The review has become increasingly necessary because

Virtually every field of science is now pervaded by a relentless crossfire in which the findings of new studies not only differ from previously established trutbs, but disagree with one another, often vehemently.

(Hunt, 1997, p1)

The role of the literature review has considerable potential for gate-keeping in professional practice, and as such standard, rigorous and replicable criteria should be 
applied to them (Cooper \& Rosenthal, 1980). The most common type of review is probably the narrative review, in which the author gathers together studies around a topic, summarising them and arriving at a qualitative conclusion. However, the opportunity for biased presentation is enormous in this type of review, through selective inclusion of studies, and subjective weighting and interpretation of the findings (Wolf, 1986; Gerbag \& Horwitz, 1988; Hunt, 1997). Such reviews also cannot examine the characteristics of studies as potential reasons for disparate results, or examine for moderating variables in the relationship being reviewed (Wolf, 1986). Furthermore, the generally implicit method of narrative review means that it cannot be reproduced and thus is of limited merit scientifically (Gerbag \& Horwitz, 1988).

Cooper and Rosenthal (1980) investigated the vulnerability of the narrative review, by presenting graduate students and faculty members with 7 articles, which were to form the basis of either statistical summation of effect size, or traditional narrative review. The participants were randomly assigned to groups and the investigators measured support for the hypothesis that "the evidence in general supports the conclusion that females are more persisient, or become more involved in tasks, than males" (ibid., p444). They found that participants using the statistical technique perceived more support and a greater magnitude of effect than did the narrative reviewers, suggesting narrative reviews could gate-keep by reflecting reviewers' own beliefs rather than that of the units of data.

Antman, Lau, Kupelnick, Mosteller and Chalmers (1992) also addressed the issue of bias in a comparison of the results of a meta-analysis of randomised controlled trials (RCTs) and the recommendations of clinical experts in narrative reviews and textbooks on the treatment of acute myocardial infarction (MI). The authors cumulatively combined the results of RCTs by year and compared the annual summary statistic to the advice contained in reviews and textbooks published the same year. They found that "review articles often failed to mention important advances in treatment or exbibited delays in recommending effective preventive measures" (ibid., p240). Ineffective treatments or treatments that were harmful also continued to be recommended by some authors. For example, 6 years elapsed between the time a meta-analysis published in the New England Medical Journal first demonstrated the reduction in mortality associated with thrombolytic therapy post MI (at $\mathrm{p}<0.001$ ), 
and the time the majority of review authors recommended it. The authors believed that this and similar intervals could not be explained by the usual publication delays. Similarly, the authors found lignocaine, which has been recommended as a prophylactic for ventricular fibrillation throughout the last 25 years in textbooks and narrative reviews, was not supported by evidence of reduction in mortality in trials.

Mulrow (1987) had earlier investigated the overall rigor of review methods in medicine by examining 50 review articles drawn from four premier medical journals ${ }^{1}$, and evaluating them against 8 criteria drawn from guidelines on information synthesis. ${ }^{2}$ Mulrow did not report how the articles were selected, but found that no review article met all criteria and only one review met 6 of the criteria. Qualitative synthesis was the commonest method of integrating the studies' conclusions, with just 3 reviews using quantitative methods of synthesis. Mulrow identified steps that review authors could take to improve the quality of information syntheses in medicine and concluded that "by using systematic methods of exploration, evaluation and synthesis, the good reviewers can accomplish the task of advancing scientific knowledge" (Muirow, 1987, p487). Though considered a seminal work in health sciences, Mulrow owes a considerable although largely unacknowledged debt to the development of research synthesis as a research activity in the social sciences, especially sociology and psychology.

\section{The Development of Research Synthesis in the Social Sciences}

The need for advances in research synthesis was signalled by Feldman (1971), in a discussion of review methods and he proposed that the method of research synthesis was analogous to that of primary research. Thus

Systematically reviewing and integrating what is nominally called the "literature" of a field may be considered a type of research in its own right - one using a characteristic set of research techniques and methods.

(Feldman, 1971, p86)

\footnotetext{
1 Annals of Internal Medicine, Archives of Internal Medicine, Journal of the American Medical Association, and The New England Medical Journal.

2 Was a specific purpose stated? Were sources and methods of citation searches identified? Were explicit criteria provided that determined the inclusion or exclusion of studies? Was methodologic assessment of validity performed? Was information systematically integrated with explication of data limitations? Was the information integrated and weighted or pooled metrically? Was a summary of pertinent findings provided? Were specific directives for new research initiatives proposed?
} 
Such reviews could act as a compendium of research relevant to a topic, simulating a broader sample than is usually possible in a single trial, and where study protocols are similar, simulate replication. Feldman concluded that a 'good integration' revealed not only how much is known, but also how little is known within a field; within this sense the integration is not an end, but a beginning. However, Feldman did not propose a method for research synthesis, although he did present an overview of potential methods and their problems. Feldman was not alone in raising the problems of reviews in psychology. Light and Smith (1971) had also identified the inadequacies of the then current methods of review and proposed that progress will only come when we are able to pool, in a systematic manner, the original data from studies (ibid., p443), rather than cumulating significance tests. They suggested the cluster approach for quantitatively synthesizing studies, a method that was similar in spirit to the soon to be developed effect size analysis, although more conservative (Glass, 1977). Within sociology, Taveggia (1974) had also identified 6 areas that were problematic for review. ${ }^{3}$ More important was Taveggia's belief that review authors had failed to understand that research results are probabilistic. The findings of a single study may not be meaningful, as they could have occurred by chance. Thus, where a number of studies have been conducted on a given topic, any apparently contradictory findings may simply be the distribution of results, chance alone having dictated the negative and positive results.

Synthetic methods for aggregating the results of independent but similar studies had received attention at various intervals throughout this century. The earliest work is probably that of Pearson in 1904, averaging the correlation between inoculation for typhoid fever and mortality in 5 studies (Cooper \& Hedges, 1994). Broadly, the methods can be grouped into three approaches: vote counting, combined tests and measures of effect size (Wolf, 1986). Vote counting involves tallying study results into positive and negative significant results, and statistically insignificant results, and determining which category has a greater than one third of the studies. This category is then the 'winner'. Vote counting has been rejected as a synthetic method principally because the method's power is low and decreases with increasing numbers of studies, thereby increasing the probability of Type II error ${ }^{4}$ (Hedges \& Olkin, 1980). The method also assumes that the size of treatment effect within each study is

${ }^{3}$ The 6 areas were selection of articles; indexing, coding and retrieval of data; analysis of comparability of findings; cumulation of findings; analysis of the resulting distributions; and reporting of results.

${ }^{4}$ Accepting the null hypothesis when it should be rejected. 
the same in the 'winning' modal category, although this is rarely the case. The use of combined tests arose out of a desire to sum the results of independent studies. Rosenthal (1978) identified seven methods for summing significance levels. Examples are adding logarithms of $p$ values (Fisher Combined Test), adding $t$ tests (Winer Combined Test) or raw or weighted z tests (Stouffer Combined Test). These tests, however, only determine whether the aggregated statistic is significant and do not provide an indication of the strength of the relationship or size of effect of a treatment. Measures of effect size address this issue. The result of each study is converted into a common metric which can then be aggregated to determine the degree that an effect is evident in a population (Wolf, 1986).

It was the seminal work of Glass (1976) that provided a statistical method for arriving at a common metric for continuous data (the $d$-index). This metric, deemed the effect size, is derived from each study by dividing the mean difference between treated and untreated subjects by the within-group standard deviation. The effect sizes are then summed and their average derived. Glass also addressed the call for an explicit methodology for the quantitative synthesis of similar, but independent studies, and coined the term 'meta-analysis' to describe this process of research synthesis. The term is now more commonly applied in the health sciences to the statistical method of quantitatively synthesizing the data. Although it is the development of effect size analysis that Glass is remembered for, Glass rejects that this was his contribution, as others in social sciences, notably Rosenthal (1978), were working on effect sizes independently of Glass. He states that it was the methodology "the whole process, from first to last that was my contribution" (Hunt, 1997, p38). The methodology Glass proposed involved formulating the research problem as a question, defining the universe of studies to sample, collecting data from the sample of studies, evaluating the quality of the data, synthesizing the data and reporting on the synthesis (Glass, 1976). This was "a rigorous alternative to the casual, narrative discussions" (ibid., p3) of narrative reviews and was the methodology of the first meta-analysis by Smith and Smith (1977), synthesizing psychotherapy outcome studies.

The methodology of research synthesis remained problematic within the social sciences, despite Glass's innovation. No widely accepted guidelines on their conduct existed. Jackson (1980) randomly sampled 36 reviews from 8 eminent journals within 
sociology, education and psychology in the mid-1970s $\mathrm{s}^{5}$ and assessed each against 6 criteria. He suggested that "relatively little thought appears to bave been given to the methods of doing integrative reviews" (ibid., p 459) and that reviews were still done less rigorously than is possible. Jackson believed that the lack of an explicit methodology for integrative reviews signified that social scientists were paying little attention to the purpose and role of reviews, and thus were probably not using as powerful methods as they could. Furthermore, Jackson opined, the absence of an explicit methodology meant that it was difficult to train beginning researchers in the performance of competent reviews, that standards for assessing the quality of reviews were nonexistent and that the accumulation of valid knowledge from previous research was consequently hindered. Cooper (1982) provided guidelines that laid out research synthesis as a process, similar to that of primary research as previous authors had suggested. Cooper also outlined the potential threats to the validity of the research synthesis for each step in the process, and perhaps more importantly, suggested how reviewers might protect against these threats.

\section{The Development of Research Synthesis in Health Sciences}

Gerbag and Horwitz (1988) suggested that there have been two methods of research synthesis in medicine, in addition to the narrative review: the methodologic analysis and the pooled analysis. Both methods have sought to resolve conflicting evidence, but in methodologic analysis, each trial is reviewed according to quality standards "to judge the scientific validity and clinical applicability of trial results" (ibid., p504). Conclusions are then drawn from the studies judged methodologically superior and thus are qualitative, although a vote counting approach may also be used (see Gifford \& Feinstein, 1969). In pooled analysis, the data from the clinical trials are combined with the goal being to achieve statistical significance when the treatment and control groups are compared. Pooled analysis was not new to medicine and reference is made earlier in this chapter to Pearson who combined the results of 5 separate studies of the correlation between inoculation for typhoid fever and mortality early this century (Cooper \& Hedges, 1994). The term meta-analysis was quickly adopted by the health sciences in the 1980s, by medicine in particular. Sacks, Berrier, Reitman, Ancona-Berk and Chalmers (1987) found 13 meta-analyses published in the 1970s,

5 Psychological Bulletin, Annual Review of Psychology, Review of Educational Research, Annual Review of Research in Education, American Sociological Review, Sociological Quarterly, Social Problems. 
and 69 published between 1980 and 1986. Dickersin and Berlin (1992) found exponential growth in the publication of meta-analyses ${ }^{6}$ in the health sciences. Computerised searching of MEDLINE ${ }^{\circledR}$ over the period 1986 to 1991 using the subject heading or text word 'meta-analysis' found growth doubled annually, with 21 published meta-analyses in 1986 and 431 by 1991. However, the term was not always applied with the precision Glass intended. It has been suggested, for instance, that Beecher's 1955 exploration of placebo was the first 'meta-analysis' in medicine, confusing any quantitative research synthesis with the method of meta-analysis (Sacks, et al., 1987; Egger \& Davey Smith, 1997). Beecher (1955) randomly identified 15 observational investigations into the placebo effect in a variety of treatments, ranging from headache, cough and sea-sickness to post-operative wound pain. Beecher summed the percentages of patients with symptoms satisfactorily relieved by placebo for each study and averaged the sum. Beecher did not address an explicit study question, define a search strategy, state inclusion or exclusion criteria, or assess the studies' methodologic quality; the single characteristic Beecher's review shared with the methodology outlined by Glass was the attempt at quantitative summation. Beecher's review was certainly a research synthesis, but it had more in common with a narrative review than with a meta-analysis and is not justified in being described as a meta-analysis. On the other hand, Kylma and Vehvilianen-Julkunen (1997), taking a systematic approach to the investigation on the ontology and epistemology of research into the concept of hope, termed their qualitative analysis a meta-analysis because it was systematic. Gerbag and Horwitz (1988) were just as imprecise and termed all the analyses in their review, whether methodologic (qualitative) or pooled analyses (quantitative), 'meta-analyses'.

The process of research synthesis in the health sciences was not the subject of the same discussion that took place in the social sciences during the 1970s, although one commentator stated in 1979

It is surely a great criticism of [medicine] that we have not organised a critical summary, by specialty or subspecialty, adapted periodically, of all relevant randomized controlled trials.

(Cochrane, 1979, p2)

\footnotetext{
${ }^{6}$ The term meta-analysis is used here in the original sense Glass intended, and does not refer to the
} statistical method of combining studies. 
When discussion did take place, it tended to focus on statistical methods for combining results, rather than the purpose and methodology of research synthesis within health sciences. Perhaps for this reason and the rather confused interpretation of 'meta-analysis', the quality of meta-analyses conducted in medicine have not been high. Sacks, et al. (1987) searched English language medical literature for published meta-analyses from 1966 to 1986, finding 86 studies that met their criteria. These syntheses were independently assessed by two raters against criteria in six major areas: study design, combinability, control of bias, statistical analysis, sensitivity analysis and problems of application. Sacks, et al. found that no synthesis received more than 14 ratings of adequate out of a total of possible 23; the mean was 7.7 (SD 2.2). In addition, only 28 percent of the papers addressed issues in all of the six major areas. The authors concluded "an ungent need exists for improved methods in literature searching, quality evaluation of trials and synthesizing of the results" (ibid., p450). This conclusion was to be supported by Mulrow's (1987) investigation into the quality of reviews, published the following month in the Annals of Internal Medicine.

However, the early critiques on the methodology of reviews in medicine did draw on the advances the social sciences had been making in research synthesis. Mulrow's criteria for evaluating review articles was drawn from the work of Light and Pillemar (1984) and Sacks, et al. (1987) referenced the work of Light and Smith (1971) and Glass (1976), although the source of their evaluative criteria was not identified. The late 1980s saw increasing attention being given to the methodology of reviews in medical literature. This did not just draw attention to meta-analysis, but advanced and adapted the methodology of research synthesis. For instance, social sciences tend to deal with continuous data, and thus the development of the $d$-index by Glass (1976) to facilitate combination of dissimilar continuous outcomes by conversion to a common metric. Quantitative data in medicine, particularly that which is meaningful to patients, tends to be dichotomous. The common metric has become a ratio of combined results for the treated and untreated groups, using any one of a number of statistical methods. Gerbag and Horwitz (1988), following a critique of 7 pooled analyses and 6 methodologic analyses published between 1969 and 1983, believed that the strengths of both methods if combined in a single review, would "enhance scientific validity and clinical applicability" of reviews (ibid., p503). This echoed a critique of meta-analysis conducted in the social sciences by Slavin, who proposed a technique he termed 'best-evidence synthesis', which combined the quantification of 
effect sizes and systematic study selection (Slavin, 1986; Slavin, 1995). Slavin suggested this approach would address the concerns of rigour that were raised by the inclusiveness of early meta-analyses in psychology and education. Gerbag and Horwitz (1988) used the noun phrase 'systematic review', in reference to the methodologic review section in a meta-analysis, a use that was first indexed in MEDLINE $^{\circledR}$ in 1976. In this review, Shaikh, Vayda and Feldman (1976) had methodologically analysed all English language studies evaluating tonsillectomy and adenoidectomy identified in a 50-year period, entitling this a systematic review. However, this was not the first use of the term; PsycLit ${ }^{\circledR}$ first indexed the term in a study's title in 1936 (Mandel, 1936). The actual origin of the term seems unknown, although Gerbag and Horwitz's (1988) suggested combination of the two methods, meta-analysis (statistically combining studies using effect size measures) and methodological analysis (quality assessment of studies) may be the origin of the systematic review in medicine. The term is now understood to mean in health sciences,

the application of scientific strategies that limit bias to the systematic assembly, critical appraisal, and synthesis of all relevant studies on a specific topic.

(Cook, Sackett, \& Spitzer, 1995, p167)

A meta-analysis was defined by the same authors as a systematic review that incorporated statistical methods to combine and summarise the results of studies (Cook, et al., 1995), although, confusingly, systematic review is also commonly applied to reviews that employ the statistical techniques of meta-analysis. Cook, et al.'s (1995) definition of meta-analysis, signifying the combined used of systematic review and meta-analysis is used throughout the following discussion on conducting a meta-analysis. Gerbag and Horwitz (1988) outlined guidelines for improving the rigour of meta-analyses, although these were not very explicit. They recommended that meta-analysis incorporate methodologic analysis, explicit criteria for grouping and comparison of studies (including the acceptable range of study attributes), and the combination of data to provide a quantitative statistic. More explicit guidelines have been published since, notably the guidelines for meta-analysis of randomised controlled trials from the Potsdam Consultation on Meta-analysis (Cook, et al, 1995), and the Cochrane Collaboration Handbook (Clarke \& Oxman, 1999). 
The Cochrane Collaboration, established in 1992, was a response to the growing need within healthcare for summative information on effective interventions. It is an international organisation that aims to prepare and maintain meta-analyses of the effectiveness of health interventions. The Collaboration is named after Archie Cochrane, an epidemiologist who recognised that information from randomised controlled trials needed to be collected into reviews that were methodologically rigorous (Cochrane, 1972). To date 663 meta-analyses have been completed with a further 624 protocols registered.

\section{Conducting a Meta-analysis}

Meta-analyses are observational investigations where the unit of analysis is not individual outcomes, but the results of the original studies. As such, meta-analyses can be likened to "an epidemiology of results" (Jenick, 1989, p39). However, meta-analysis is potentially subject to the same biases that affect all observational studies. For a meta-analysis to be reliable and valid, it must rely upon the quality of the included studies and, as importantly, the methodology of the meta-analysis itself (Cook, et al., 1995). The Potsdam Consultation on Meta-analysis sought to improve the conduct of meta-analyses within the constraints of "the real world of imperfect studies" (ibid., p167). The participants identified the need to describe and disseminate good review practice and aimed to do this through the production of guidelines on the conduct of meta-analyses of randomised controlled trials. These guidelines were published by Cook, et al. (1995) and, along with the NHS Centre for Review and Dissemination's (1996) guides for systematic review and the Cochrane Handbook (Clarke \& Oxman, 1999), are used to inform the following discussion. As befits the conduct of any research, the three guidelines recommend that a protocol be developed for the metaanalysis and this should pose a research question specifying the study population, intervention and outcomes. The protocol should also specify the methods used to search for all potentially relevant data, the selection criteria, the methodological assessment of quality and the methods used to analyse the data. The protocol should also specify any sub-group hypothesis testing a priori.

\section{Search Strategy and Study Selection}

The meta-analysis when reported should specify the strategy used to identify studies 
for review. This should outline the databases used, other sources of studies, language restrictions and attempts to identify unpublished studies. It has been suggested that there is a bias on publication towards studies that have a positive effect over those that report no effect (Rennie \& Flanagin, 1992), and that English language reports tend to favour positive reports (Egger, Zellweger-Zahner, Schneider, Junker, Lengeler \& Antes, 1997). Scherer, Dickersin, and Langenberg (1994) suggested that up to 50 percent of studies do not reach publication in journals. Although inclusion of unpublished studies is contentious (Cook, et al., 1993), reliance on published studies and, or, only those published in English may over-estimate the effect of a treatment (Egger \& Davey Smith, 1995). The publication of the search strategy allows readers of reviews to assess the efforts of the authors of the meta-analysis have made to overcome publication and language bias.

Reports of meta-analyses should identify the selection criteria for inclusion and exclusion of studies that address the research question. These criteria should be based upon the population, intervention(s), outcomes and study design specified in the protocol. Reasons for exclusion should be presented, allowing the reader to assess the reproducibility of the study selection. The process of selection should be conducted by at least two reviewers in order to minimise selection bias, with a process for arbitration of disagreements between the reviewers. Once the included studies have been selected, a methodologic assessment of the studies' quality should be conducted. Moher, Pham, Jones, Cook, Jadad, Tugwell and Klassen (1998), in a re-analysis of 11 randomly selected meta-analyses involving $127 \mathrm{RCTs}$, found that unblinded trials were associated with an increased estimate of benefit of 34 percent when compared with blinded trials. A similar over-estimate of benefit (37 percent) was produced when trials employing random allocation were compared with those that did not. However, it is important that the quality assessment not confuse methodologic quality with quality of reporting. For this reason, quality assessment should be limited to assessing procedures for minimising threats to internal validity e.g. method of allocation and concealment, blinding, objective criteria for outcomes, completeness of follow-up and handling of departures from the original protocol. Moher, Jadad, Nichol, Penman, Tugwell and Walsh (1995) identified 25 scales and 9 checklists to assess the 'quality' of studies based on generally accepted criteria. Only one tool had been validated (Jadad, 1998). If a tool is used, the scoring system should be transparent. However, the Cochrane Collaboration does not recommend the use 
of quality scoring systems (Clarke \& Oxman, 1999). The quality assessment provides information that may explain heterogeneity or be used for the conduct of sensitivity analysis.

\section{Data Extraction and Synthesis}

The data extraction and synthetic phase of a meta-analysis should be explicit, reproducible and minimise bias. To this end the data extraction should be conducted by independent reviewers with a standardised coding form and methods for resolution of disagreements between the reviewers should be reported (NHS Centre for Reviews and Dissemination, 1996). It is also recommended that, where there are ambiguities in the published studies, the original investigators be contacted for clarification. When the data is sparse, of low quality or too heterogenous to proceed with a statistical aggregation, the reviewers should perform a narrative analysis. Where statistical aggregation is possible, all the relevant and clinically useful measures should be used. The goal of effect size measures is to obtain a number free of the original unit of measure in order to index "the degree of departure from the null bypothesis of the alternative hypothesis" (Cohen, 1977, p20). Two measures of effect size are generally utilised for categorical or binary data, where one outcome is preferred and the other is in some sense undesirable. These are the odds ratio (also known as the crossproduct ratio and abbreviated to $\mathrm{OR}$ ) and the relative risk (also known as the rate ratio and abbreviated to $R R$ ). The relative risk is the ratio of two probabilities and is calculated from $\mathrm{X}_{1} / \mathrm{X}_{2}$, where $\mathrm{X}_{1}$ and $\mathrm{X}_{2}$ are the average effect for the two comparison groups. The odds ratio is the ratio of odds estimable for the outcome in each group, and is calculated from $\left(\Pi_{1} / \Pi_{2}\right) /\left(\Pi_{3} / \Pi_{4}\right)$, where $\Pi_{1}$ and $\Pi_{3}$ are the numbers with the outcome of interest in the two groups and $\Pi_{2}$ and $\Pi_{4}$ are the numbers without the outcome of interest in the two groups.

The odds ratio is the measure most commonly used in health-related meta-analysis primarily because it is estimable from a variety of study designs. It also has statistical properties of practical importance; for instance it can assume any value between 0 and $\infty$, no matter what the value of the two probabilities being compared. Additionally, when the proportions being compared are small, the odds ratio approximates the relative risk (Cooper \& Hedges, 1994). This feature makes the odds ratio acceptable in studies where the event rate is low, as in large pharmaceutical trials 
examining differences in mortality when two therapies are being compared. However, in trials where the event rate is high, the odds ratio can considerably overestimate the relative risk, potentially leading to precipitate conclusions regarding the effectiveness of a therapy. Trials investigating the effectiveness of therapies in ulcer healing are an example of the latter, as healing is a relatively common event. Unfortunately, if the chances are high that a participant will experience the outcome under study, many values for the relative risk are mathematically impossible. For instance if the mean event rate in one group is 0.4 (or 40 percent), only values for the interval $0 \leq R R \leq 2.5$ are possible. Tests for between-study homogeneity can consequently over-estimate heterogeneity ${ }^{7}$ as a result of variation in relative risk, leading to a conclusion of significant heterogeneity where heterogeneity is not significant. This constraint does not characterise the odds ratio (Cooper \& Hedges, 1994).

It is reasonable to combine studies that are conducted on similar populations with similar study designs, entry criteria and treatment protocols. Such groups of studies are rare, however. More commonly, studies use different entry criteria, definitions of disease, methods of measuring the outcome and variations in treatment (Dickersin $\&$ Berlin, 1992). Groups of studies that vary in design, population and protocols are likely to produce variance in the estimation of the treatment effect. The issue is whether the variation (or heterogeneity) in study results is greater than is compatible with the play of chance (Thompson, 1994). An assumption underpinning pooling of effects is that each study provides an estimate of effect size that is representative of the population effect size (Wolf, 1986; Bailey, 1987). This assumption and the generalisation of a pooled statistic to a population of future patients have been described as a leap of faith (Thompson \& Pocock, 1991). Combining studies and generalising the pooled statistic may be appropriate if a series of studies provide a homogenous estimate of the effect size, suggesting the studies are testing the same hypothesis. However,

if these studies are beterogeneous, then the question of whether each study is testing the same hypothesis arises. Heterogeneity provides a warning that it may not be appropriate to combine and synthesize all the study results in a meta-analysis

(Wolf, 1986, p42)

7 Statistical heterogeneity is variation in study results, which may be caused by clinical or 
Heterogeneity of studies is substantial when between study estimates of treatment effect vary above that expected by sampling error or chance (Dickersin \& Berlin, 1992; Thompson, 1994; Sutton, Jones, Abrams, et al., 1999). When the studies differ substantially, the pooled effect size may not be representative of the components making up the average (Dickersin \& Berlin, 1992). Heterogeneity usually tests the null hypothesis, that there is no significant heterogeneity among the study results, using chi-square statistics. A chi-square statistic has on average a value equal to its degrees of freedom (Thompson \& Pocock, 1991). The criteria for rejecting the null hypothesis is the subject of debate, but it has been recommended that a less stringent than normal criteria apply as the power of the chi-square to detect heterogeneity is not strong (Fleiss, 1986; Bailey, 1987). A significance threshold of $\mathrm{p} \leq 0.10$ is the conventional criteria for rejecting the null hypothesis when testing heterogeneity (Bailey, 1987). However, it should be noted that an insignificant test result couldn't be interpreted as evidence of homogeneity, as the low power of the test may not detect even moderate heterogeneity.

The decision to pool treatment effects should not rest on the results of a statistical test alone, although Thompson and Pocock (1991, p1352) notes an investigator should feel "bappier to ignore problems of clinical beterogeneity if direct evidence of statistical beterogeneity is lacking". The guiding principle should be to investigate the impact of clinical and methodological differences, rather than rely on statistical testing alone (Bailey, 1987; Thompson \& Pocock, 1991). This can be achieved by the use of sensitivity analyses (removing specific studies meeting set criteria and exploring the impact of this on the pooled effect size). Sensitivity analyses test the robustness of results relative to key assumptions and decisions made about the data. Generally, sensitivity testing is conducted where there may be any ambiguity about the trials' methodology, uncertainty about whether the trials meet inclusion and exclusion criteria, test missing data and determine the vulnerability of the results to small shifts in the number of events between the treatment and control groups.

If all studies were similar in design, they would differ "only by virtue of having used just a sample of observations from the total population" (Thompson \& Pocock, $1991 \mathrm{p} 262$ ) and the effect sizes would differ from that of the true population only because of sampling

methodological differences in trials (Thompson, 1994). 
error (also known as conditional variance). However, it is rarely the case that studies are perfect replicas of each other. Most often studies differ methodologically. What is at issue is described as one of the most subtle and difficult problems in research synthesis; the specification of the universe ${ }^{8}$ to which the researcher wishes to generalise, which in turn determines how the results of the synthesis are interpreted (Cooper \& Hedges, 1994).

Two models for combining effect sizes have been advanced: the fixed effects model and the random effects model. Essentially the two models are distinguished by how the definition of the universe of studies to which the sample of studies is generalisable. A fixed effects model assumes that

The universe to which generlizations are made consists of ensembles of studies identical to those in the study sample except for the particular people (or primary sampling units) that appear in the studies.

(Cooper \& Hedges, 1994 p30).

A random effects model assumes the studies are drawn from a sample of a collection or population of studies. The universe to which generalisations are made is the population of studies from which the sample is drawn, rather than all hypothetically possible studies. The studies in this universe differ from the sample studies along two dimensions: study characteristics and effect size, and the sampling of people into the individual studies, resulting in variations in the observed effect sizes. Cooper and Hedges (1994, p31) note that

The generalization is not [sic], as it was in the fixed effects case, to a universe consisting of ensembles of studies with corresponding members of the ensembles having identical characteristics and effect size parameters. Instead the studies in the study sample (and their effect size parameters) differ from those in the universe by as much as might be expected as a consequence of drawing a sample from a population.

The question of whether to use a fixed or random effects model in combining effect sizes must be made on the degree to which the studies are similar. Consideration

\footnotetext{
8 The universe is the hypothetical collection of studies that could be conducted and about which the synthesis will generalise. The study sample is the collection of studies that have been assembled for use in the systematic review.
} 
should be given to the features of the participants and to the studies' methodologies, as well as a statistical measure of heterogeneity.

The synthesis of perfectly alike studies might use a simple fixed effects model, with the estimation of population effect size being derived from the average of the observed studies' effect sizes. A simple fixed effects model would give each study the same weight within a synthesis. However, if studies vary by sample size, smaller studies would have the same weighting as larger samples, despite studies with larger samples being acknowledged as being more precise in determining a treatment effect (Cooper \& Hedges, 1994). A more complex fixed effects model assumes the ensemble of studies is generalisable to the hypothetical universe of studies, but weights the studies on the basis of their relative sample sizes, with more weight being given to studies with larger samples. Weighting is also used in the random effects model, although the smaller studies are given greater weights than in the fixed effects model. This acknowledges that the results of smaller studies represent random variation within the universe of studies, rather than lower precision. The random effects model provides more conservative estimates of the "significance of the average effect sizes over studies in the presence of unexplained beterogeneity" (Cooper \& Hedges, 1994, p275) than the fixed effects model. It is for this reason the Cochrane Collaboration recommends that the decision regarding which model for combination of effect sizes is to be used should be made prior to the combination of studies (Oxman, Cook, \& Guyatt, 1994), thus avoiding the temptation to present the findings using the more favourable model.

\section{Interpretation and Presentation of Results}

The reporting of results should include evidence tables that specify the design, sample size, treatment effect, outcome measures and information likely to have an impact on the internal validity of the study. 'The summary data should be shown graphically as well as reported in the text, and clinically relevant measures of effectiveness, such as 'number needed to treat' should be stated. ${ }^{10}$ The results should also report confidence intervals at a specified level of probability. Consideration

\footnotetext{
${ }^{9}$ Method of allocation, allocation concealment, blinding, completeness of follow-up and treatment of withdrawals.

${ }^{10}$ Number needed to treat (NNT) to cause one more beneficial event in the treatment than in the control group. It is calculated by determining the inverse of the absolute difference between the treatment and control groups.
} 
should be given to publication bias when examining the results. A funnel plot plots the precision of each study against effect size. Where publication bias is absent, the expectation is that the plot should represent an inverse funnel with the more precise studies at the peak and less precise studies at the base of the funnel. An asymmetrical funnel plot suggests the presence of publication bias. However, it may also suggest bias due to the usually poorer methodological quality of small studies, and for this reason a funnel plot should only be used to draw attention to a potential problem. The power of this method is at its most limited when a review consists of a few small studies; for this reason it is not part of the publication of a Cochrane meta-analysis (Clarke \& Oxman, 1999).

When interpreting the results, the reviewers must be mindful of the needs of clinical practice and thus must situate the interpretation within current healthcare practices. It would be of little use to practitioners to report results of outmoded practices, unless this was to confirm they are ineffective. The interpretation should call upon other available evidence to better make sense of the inferences about the direction of effect; human physiological data and observational data may support the likelihood of a causal relationship. The interpretation should also consider dose-dependent responses where relevant, and adverse effects associated with the treatment. When considering the strength of inferences about the effectiveness of an intervention, the report should also consider the methodologic limitations of the included studies and the meta-analysis. The report should consider the implications of the meta-analysis for clinical practice, although these implications should be limited to those that can be clearly derived from the evidence, and should not be directive of practice. Finally the report should propose a future research agenda that addresses both clinical and methodologic issues.

\section{Conclusion}

Research synthesis serves a variety of purposes; in the increasingly complex world of healthcare interventions, the primary purpose of research synthesis is to advance knowledge through the accumulation of all studies relevant to a specific question, in order to test a hypothesis or generate an answer to the question. Advances in research synthesis are owed to developments in the social sciences during the 1970s and early 1980s. The health sciences, and medicine in particular, have drawn on these developments, as it became apparent just how vulnerable narrative reviews were to 
bias. The nexus of this vulnerability and the need to ensure clinical practice is effective has ensured the place of research synthesis as a legitimate research activity. The Cochrane Collaboration, with its clear guidelines for methodologic analysis and quantitative pooling, through meta-analysis, have provided a home for the output of this research activity in health, as well as a methodologic sanctuary for the science of meta-analysis. 


\section{Chapter 3. METHOD}

This meta-analysis was conducted as part of a larger review, for use in an explicit evidence-based clinical practice guideline on the care of patients with chronic leg ulcers. It represents an a priori specified subgroup analysis to investigate the question

\section{Is pentoxifylline and compression more effective in bealing venous leg ulcers than compression and placebo?}

The principal reviewer (Andrew Jull; AJ) was assisted in the larger review by two other reviewers, Associate Professor Bruce Arroll (BA), University of Auckland and Dr Jill Waters (JW), Auckland Healthcare Services Ltd. The roles of the principal reviewer and co-reviewers are indicated throughout this chapter by use of their respective initials where the tasks were shared (See Appendix 1). Where no co-reviewer is indicated, the principal reviewer was solely responsible for the task.

\section{Search Strategy}

The CENTRAL registers of the Cochrane Wounds Group and the Cochrane Peripheral Vascular Diseases Group were searched for randomised controlled trials and controlled clinical trials of pentoxifylline in the treatment of venous leg ulcers. In addition, the manufacturer of pentoxifylline (Hoechst) was contacted via the New Zealand distributor for details of any unpublished or ongoing studies. Articles reviewing the use of pentoxifylline in the treatment of ulceration were obtained and citations within these reviews and the primary studies were scrutinised to identify any additional studies.

The two Collaborative Review Groups (CRG) liaise to avoid duplication of searches. The Wounds CRG is responsible for reviewing interventions for venous ulceration. The Peripheral Vascular Diseases CRG is responsible for reviewing interventions for arterial and mixed arterial/venous leg ulceration. The CENTRAL registers contain references from comprehensive searches of electronic databases, handsearches of relevant journals and conference proceedings, and contacts with product companies and experts in the field. In addition the citations of obtained papers are scrutinised by 
the CRGs for additional studies. The search strategy for each respective CRG is published on the Cochrane Library and has been reproduced in Appendices 2 and 3.

\section{Criteria for Inclusion of Studies}

Types of participants: Studies which involved adult participants of any age and in any care setting, described as having 'venous leg ulcers' or equivalent (stasis ulcers, gravitational ulcers, varicose ulcers or post-thrombotic ulcers) were included. The criterion for diagnosis of venous ulceration was identified where available, but the lack of internationally agreed diagnostic criteria meant that this could not be used as an inclusion or exclusion criterion. Studies which described inclusion of participants with venous ulceration have implicit a clinical assessment of aetiology and were included in the review unless other criteria led to their exclusion. Sensitivity analyses were conducted to determine the impact of trials that relied on clinical assessment alone without further non-invasive testing (such as Doppler ultrasonography) to determine aetiology. Trials that recruited participants with other causes of ulceration, such as arterial, diabetic or rheumatoid ulceration, were included only if the results of those participants with venous ulceration were presented separately.

Types of intervention: Only trials that compared pentoxifylline and compression therapy with a placebo and compression therapy in the treatment of chronic venous ulceration were included.

Types of outcome measures: The outcome measures must have been objectively measured. The primary outcome measures for this investigation were measures that could be dichotomised, such as time to complete healing, proportion of ulcers healed within the trial period, or rate of change in ulcer area within the trial period. The outcome of healing must have been operationally defined as $100 \%$ complete reepithelialisation of the ulcer or specified as complete healing.

Types of studies: Trials were only included if the allocation of participants to therapies was described as 'randomised' or an actual random allocation schedule was described. Studies that employed pseudo-randomisation techniques, such as allocation by birth date, patient number or alternate weeks, would not have been included, but no studies described using such strategies. 


\section{Initial Selection of Studies}

The results of the searches of the CENTRAL register for each group were forwarded to the prinicipal reviewer in text file formats via email. The manufacturer of pentoxifylline (Hoechst) also forwarded a list of studies, although no studies not previously identified by the Cochrane groups were identified by this means. Print copies of each text file were examined by the author and a co-reviewer (BA) for relevant studies. These lists were compared and agreement was reached regarding studies identified as candidates for further examination. These studies were extracted electronically from the various text files and compiled into a single 'final list' text file. All studies on this list were obtained for further examination. Review articles were also extracted from the list, obtained and scrutinised for studies relevant to the investigation.

\section{Translation of Studies}

Translations of the methods, results and any tables, figures and graphs were obtained for three articles (Schürmann \& Eberhardt, 1986; Apollonio \& Angeletti, 1992; Herdy, Thomaz, Souza, Robadey \& Rodolfo 1997). The translation of Schürmann and Eberhardt (1986) was obtained through the Cochrane Collaboration Sub-fertility and Dysmenorrhoea Review Group. Dr. Jorg Neumann translated the methods and results sections, and captions to the graphs from the original German. The translations of Apollonio and Angeletti (1992) and Herdy, et al. (1997) were funded by the New Zealand Centre for Evidence Based Nursing, and commercial translators provided the translation from Italian (European Translation Services) and Portuguese (Language Links Ltd).

\section{Selection of Included Studies}

The principal reviewer $(\mathrm{AJ})$ and a co-reviewer $(\mathrm{BA})$ each independently reviewed the ten studies obtained from the final list of the initial selection, studies identified from review articles and studies identified from citations of obtained studies. Each study was coded using the Inclusion/Exclusion Criteria Coding Sheet (See Appendix 4) and an opinion was formed by each reviewer as to whether the studies met the inclusion criteria. The two reviewers met to discuss the inclusion or exclusion of each study. A 
study was only included if both reviewers were in agreement that it met the inclusion criteria. Where disagreement existed, it was resolved by the decision of the third reviewer (JW), or the advice of the Cochrane Wound Review Group.

\section{Data Extraction}

Data was extracted from the included studies by the principal reviewer using a coding; form (See Appendix 4). 'The coding form was constructed drawing on the protocol guiding the meta-analysis and data extraction forms used previously by the Cochrane Wounds CRG. The principal reviewer (AJ) piloted the coding form in order to determine the adequacy and accuracy of data extraction, and adjusted the coding form as appropriate. The coding form for each included study was scrutinised by a second reviewer (JW) to ensure accuracy and completeness of data collection. Disagreements were either resolved in discussion, or by the arbitration of another coreviewer $(\mathrm{BA})$. The decision concerning the comparisons to be made was the result of a discussion between the principal reviewer and the co-reviewers.

\section{Data Synthesis}

In order to determine what model should be used to combine the studies, they were examined for methodological and clinical heterogeneity. All studies were randomised controlled trials, used blinding and compared the treatment to a matching placebo. Similarly, all trials attempted to include only patients with venous insufficiency, although the exact method by which the diagnosis was determined varied. Schürmann and Eberhardt (1986) did not specify how the diagnosis of venous insufficiency was determined, although the title of the study indicated only people with varicose or post-thrombotic ulcers were included. However, there was an overlap in the methods used by the other studies, with objective assessments utilised as well as clinical history in all studies (Table 3, see page 38). With the exception of Dale, Ruckley, Harper, Gibson, Nelson and Prescott (1999), who reported the median Ankle Brachial Index (ABI), and Schürmann and Eberhardt (1986), who did not report the mean or median Ankle Brachial Index for their participants, the participants in the other trials (Colgan, Dormandy, Jones, Schraibman, Shanik, \& Young, 1990; Barbarino, 1992; Falanga, Fujitani, Diaz, Hunter, Jorizzo, Lawrence, Lee, Menzoian, Tretbar, Holloway, Hoballah, Seabrook, McMillan, \& Wolf, 1999) all 
had a mean $\mathrm{ABI}$ greater than 0.8 , indicating the absence of significant arterial insufficiency (Stoffers, Kester, Kaiser, Rinkens, Kitslaar \& Knotterus, 1996). The mean $\mathrm{ABI}$ (control: treatment) for the three studies was $0.85: 0.84,1.06: 1.05$, and 1.1:1.1 respectively.

The populations included in each study were also examined for variation that might influence the outcome of data synthesis. Schürmann and Eberhardt (1986) provided no demographic data, apart from mean ulcer size, although the authors stated age,

Table 3: Diagnostic assessments utilised lo detemine venons aetiology in included studies

\begin{tabular}{|c|c|c|c|c|c|}
\hline & $\begin{array}{l}\text { Clinical } \\
\text { Itistory }\end{array}$ & $\begin{array}{l}\mathrm{ABI} \\
>0.8\end{array}$ & $\begin{array}{l}\text { Venous } \\
\text { Reflux }\end{array}$ & $\begin{array}{l}\text { Venous } \\
\text { Pressure }\end{array}$ & $\begin{array}{c}\text { Valve } \\
\text { Incompetent }\end{array}$ \\
\hline \multicolumn{6}{|l|}{ Schümann (1986) } \\
\hline Colgan (1990) & $\checkmark$ & $\checkmark$ & & & \\
\hline Barbarino (1992) & & $\checkmark$ & $\checkmark$ & $\checkmark$ & $\checkmark$ \\
\hline Dale $(1999)$ & $\checkmark$ & $\checkmark$ & $\checkmark$ & & \\
\hline Falanga (1999) & $\checkmark$ & & $\checkmark$ & & \\
\hline
\end{tabular}

height, weight and history of ulceration were comparable. Age, sex ratio (male:female), mean ulcer size and mean ulcer duration were the population charactcristics examined (Tables + and 5, see page 39). The within-study mean ages (control: treatment) were similar in all other trials as was the between-study variation, with the studies reflecting the association between ageing and prevalence of venous ulceration (Baker, et al., 1991). The within-study ratio of male to female participants

Table 4: Characteristics of populations of included studies - Mean age and sex ratio. $C T L=$ control, PTX=penloxifylline

\begin{tabular}{|l|c|c|c|c|} 
& \multicolumn{2}{c}{$\begin{array}{c}\text { Mean } \\
\text { Age }\end{array}$} & \multicolumn{2}{c|}{$\begin{array}{c}\text { Mex Ratio } \\
\text { Malegremale }\end{array}$} \\
\hline & CTL & PTX & CTL & P'TX \\
\hline Schürmann (1986) & - & - & - & - \\
\hline Colgan (1990) & 70.2 & 71 & $1: 2.0$ & $1: 3.2$ \\
\hline Barbarino (1992) & 64.8 & 63.2 & $1: 1.0$ & $1: 2.0$ \\
\hline Dale (1999) & 68.1 & 70.8 & $1: 1.63$ & $1: 2.4$ \\
\hline Falanga (1999) & 60.4 & 58.4 & 1.0 .78 & 1.0 .8 \\
\hline
\end{tabular}

appears to vary, although each study has reported non-significant variation. On the whole, there were greater numbers of fomale participants, reflecting the greater incidence of venous ulceration in women in prevalence studics (Nclzén, Bergquist, \& Lindhagen, 1994). The exception to this is Falanga, et al. (1999), who reported a greater number of male that female participants were included in their trial. This may reflect a sub-population that raries from that of fiuropean studies and could be cause 
for caution in determining the statistical model with which to combine the studies. However, it is worth noting that Falanga, et al. had a similar relative risk to Dale, et al. (1999), 1.18 compared to 1.23, despite Dale, et al. having a greater ratio of female participants. This suggests that the sex ratio may have little systematic impact on the trials' outcomes.

The within-study variations for mean ulcer size were similar $\left(0.4 \mathrm{~cm}^{2}-3.1 \mathrm{~cm}^{2}\right)$. There was between-study variation, with the range for controls being $4.7 \mathrm{~cm}^{2}-14.5 \mathrm{~cm}^{2}$, and $2.5 \mathrm{~cm}^{2}-14.1 \mathrm{~cm}^{2}$ for the treatment groups. The exception was Schürmann and Eberhardt (1986), who reported a within-in study mean ulcer size that favoured the treatment group. The between-study variation seems likely to have had little systematic impact on studies' results as the relative risk for the two studies of similar size (Colgan, et al., 1990; Falanga, et al., 1999) was 2.12 and 1.18 respectively. This was despite one having the smaller mean ulcer sizes and the other considerably larger mean ulcer sizes. However, this may be further cause for caution in combining the

Table 5: Characteristics of populations of included studies - Mean ulcer size and duration. $\dagger$ Dale, et al. (1999) reported the median maximum ulcer diameter, which was $2.9 \mathrm{~cm}$ (control) and $2.7 \mathrm{~cm}$ (treatment) and the median ulcer duration. $\neq$ Months.

\begin{tabular}{|l|c|c|c|c|} 
& \multicolumn{2}{c}{$\begin{array}{c}\text { Mean } \\
\text { Ulcer Size }\end{array}$} & \multicolumn{2}{c|}{$\begin{array}{c}\text { Mean Ulcer } \\
\text { Duration }\end{array}$} \\
\hline & CTL & PTX & CTL & PTX \\
\hline Schürmann (1986) & $5.4 \mathrm{~cm}^{2}$ & $2.5 \mathrm{~cm}^{2}$ & - & - \\
\hline Colgan (1990) & $4.7 \mathrm{~cm}^{2}$ & $5.2 \mathrm{~cm}^{2}$ & 9 & 6 \\
\hline Barbarino (1992) & $14.5 \mathrm{~cm}^{2}$ & $14.1 \mathrm{~cm}^{2}$ & 26.3 & 26.3 \\
\hline Dale (1999)† & - & - & 4 & 6 \\
\hline Falanga (1999) & $9.6 \mathrm{~cm}^{2}$ & $11.4 \mathrm{~cm}^{2}$ & 6 & 6 \\
\hline
\end{tabular}

studies. The mean ulcer duration was reported in three studies (Barbarino, 1992; Colgan, et al., 1990; Falanga, et al., 1999), whilst Dale, et al. (1999) reported the median ulcer duration. If the sample distribution is not skewed, the median should closely approximate the mean. The mean was similar in all cases, with exception of Barbarino (1992). The participants in this study had a considerably longer history of ulceration. This reflects the inclusion criteria (ulcers of greater than 2 years duration), and the study's aim to investigate refractory ulcers. This may be further reason for caution when combining the studies.

In summary, the trial methodologies, as far as can be determined, were substantially similar, as were the characteristics of the studies' samples. However, some differences in the samples and the study methods suggested caution be adopted in 
combining the studies. Therefore, the studies were combined using a fixed effects model and the robustness of the pooled result was tested by combining the studies using a random effects model. Sensitivity analyses were also performed, to examine the impact of clinical heterogeneity. The data was synthesised by the principal reviewer using RevMan Version 4.03 (Cochrane Collaboration, 1999). RevMan is public domain computer software available over the Internet from Cochrane file transfer sites (www.cochrane.org.uk). It was developed by the Cochrane Collaboration to support the performance of meta-analyses and contains word processing, statistical and reference management components. The statistical analysis and Peto tables for this investigation were performed by the principal reviewer using RevMan. The Peto tables were exported into Microsoft Word as bitmaps using Microsoft Paint. The variation in event rates (the control group event rates varied from 16 percent to greater than 50 percent) was likely to introduce an overestimation of heterogeneity, if relative risk was used as the summary statistic. ${ }^{1}$ However, relative risk was used to report results in the knowledge that it more accurately represents risk differences when study event rates are high, and that an insignificant test for heterogeneity will represent greater between-study homogeneity than the test result actually suggests when relative risk is used.

\footnotetext{
${ }^{1}$ See page 28 for a discussion of heterogeneity and relative risk.
} 


\section{Chapter 4. DATA COLLECTION \& SYNTHESIS}

Ten separate studies concerning venous ulcers and pentoxifylline were selected from the studies retrieved from the central registers of the Cochrane Peripheral Vascular Diseases and Wound Review Groups as deserving further investigation. No new studies were identified from the lists provided by the manufacturer of pentoxifylline (Hoechst). One further study was identified after the search had been completed (Falanga, et al., 1999). The unpublished trial from 1997 was referenced in a review article (Falanga, 1999) brought to the attention of the principal reviewer by the Cochrane Wounds Group. The trial's authors were contacted for data from the study; in actuality, the trial had just been published, but was not yet referenced on the electronic databases, or available in. Australasian libraries. An imprint of the article was faxed by the authors to the principal reviewer.

Ten of the eleven studies were described as randomised controlled trials; one study (Angelides \& Weil von der Ahe, 1989) did not have a control group and was not assessed further. Five studies (Apollonio \& Angeletti, 1992; Arenas \& Atoche, 1988; Herdy et al, 1997; Mirshahi, et al., 1995; Weitgasser, 1983) did not meet the criteria for inclusion in this meta-analysis. Reasons for exclusion included: subjective outcome assessments, compression therapy not being used, physiological outcomes reported, and the comparison group not being a placebo (or no therapy). The excluded studies and the reason for each study's exclusion are detailed below.

\section{Details of excluded studies}

Weitgasser (1983): Double-blind randomised controlled trial; allocation concealment not described; participants received pentoxifylline 1200mg daily or placebo; 60 participants, 30 in each arm; participants received treatment for minimum of 6 weeks and maximum of 8 weeks; outcome healing - assessed as good (complete closure or considerable reduction in ulcer size and reduction in symptoms), satisfactory (gradual healing marked by islands of granulation tissue and marginal epithelialisation, and reduction in symptoms), no change, or worse; outcome favoured treatment (good response $20 / 30$ versus $7 / 30$ in placebo). Reason for exclusion: outcome assessment subjective; compression therapy not used. 
Arenas and Atoche (1988): Double-blind randomised controlled trial; allocation concealment not described; participants received pentoxifylline $1200 \mathrm{mg}$ daily or placebo; 30 participants, 18 in treatment arm and 12 in the control arm; withdrawals excluded from analysis ( 3 in treatment arm, 2 in control arm); duration of treatment not described; participants followed up for 6 months; outcome healing - assessed as healed (95-100\% improvement), significant improvement (60-94\% improvement), improvement (30-59\% improvement), no change, or worse; healing and significant improvement favoured the treatment arm (58.4\% versus 30\%). Reason for exclusion: outcome assessment subjective; compression therapy not used.

Apollonio and Angeletti (1992): randomised controlled trial; allocation concealment not described; treatment blinding not described; participants received standardised treatment (compression bandaging and topical therapy) in addition to pentoxfylline $1200 \mathrm{mg}$ daily or defibrotide $800 \mathrm{mg}$ daily; 23 participants (25 lesions), 12 in defibrotide arm and 11 in the pentoxifylline arm; duration of treatment not reported; participants followed up for 6 months; outcome healing - favoured defibrotide arm at 12 weeks (12/13 versus 10/12 ulcers healed), all ulcers healed in both groups at 24 weeks. Reason for exclusion: comparison group not a placebo.

Mirshabi, et al. (1995): Double-blind randomised controlled trial; allocation concealment not described; participants received pentoxifylline $1200 \mathrm{mg}$ daily or placebo; 10 participants 4 participants in treatment arm and 6 participants in the control arm; participants received treatment for 2 months; punch biopsies taken from the centre of each ulcer prior to treatment and at 2 months; outcome - fibrin and elastase reduced in treatment arm. Reason for exclusion: outcome not meaningful to patients; physiological assay of effectiveness of pentoxifylline in reducing fibrin production.

Herdy, et al. (1997): Randomised controlled trial; allocation concealment not described; treatment blinding not described; participants received standardised therapy (leg elevation, dietary advice, daily dressing with saline, povidine-iodine and liquid paraffin) in addition to pentoxifylline $1200 \mathrm{mg}$ daily or placebo; 12 participants, 6 in each arm; no withdrawals; participants received therapy for 12 weeks; mean ulcer size favoured control at baseline $\left(9.53 \mathrm{~cm}^{2}\right.$ versus $\left.6.0 \mathrm{~cm}^{2}\right)$; outcome healing - no ulcers completely healed at trial's conclusion, mean reduction in ulcer size favoured 
treatment arm $\left(2.21 \mathrm{~cm}^{2}\right.$ versus $\left.0.62 \mathrm{~cm}^{2}\right)$. Reason for exclusion: compression therapy not used.

\section{Description of included studies}

Detailed tables of the characteristics of included studies are reported in Appendix 6. Five studies (Barbarino, 1992; Colgan, et al., 1990; Dale, et al., 1999; Falanga, et al, 1999; Schürmann \& Eberhardt, 1986) met the criteria for inclusion in this metaanalysis. Details regarding one trial (Dale, et al., 1999) have been extracted from an additional report (Prescott, Nelson, Dale, Harper \& Ruckley, 1998). The numbers of participants included in the trials ranged from 12 to 200. Three trials were 2 arm parallel group trials (Barbarino, 1992; Colgan, et al., 1990; Schürmann \& Eberhardt, 1986). A fourth trial was a stratified factorial trial with three strata, one of which only included participants with a pure venous aetiology (Dale, et al., 1999). Within the venous strata, three treatments (pentoxifylline versus a placebo, four layer compression bandaging versus single layer bandaging, hydrocolioid dressing versus a knitted viscose dressing) were tested, randomly allocated between 2 parallel groups (see Figure 3). The fifth trial was a three arm parallel group trial comparing $1200 \mathrm{mg}$ and $2400 \mathrm{mg}$ doses of pentoxifylline with a placebo (Falanga, et al., 1999).

All participants in the trials were deemed to have venous ulceration and, with the exception of Schürmann and Eberhardt (1986), all trials reported how this determination was made. The methods of diagnosis were generally similar. Colgan, et al. (1990) relied upon clinical appearance and exclusion of significant arterial insufficiency by calculation of the ankle-brachial index (ABI), which is also known as the ankle-arm index or ratio, the Winsor Index, and the ankle-brachial pressure index. This is a ratio of systolic blood pressure measured at the ankle divided by the systolic blood pressure measured at the upper arm. A ratio of greater than 1.0 is considered indicative of an absence of arterial pathology. There is general agreement that a ratio of less than 0.8 to 0.9 indicates arterial insufficiency, although there has been only one well conducted diagnostic study within a general practice setting to test this (Stoffers, et al., 1996). Barbarino (1992) relied on diagnosis by calculation of the $\mathrm{ABI}$, determination of venous pressure at the ankle, valvular incompetence and the presence of venous reflux, all using Doppler ultrasound. Dale, et al. (1999) used 


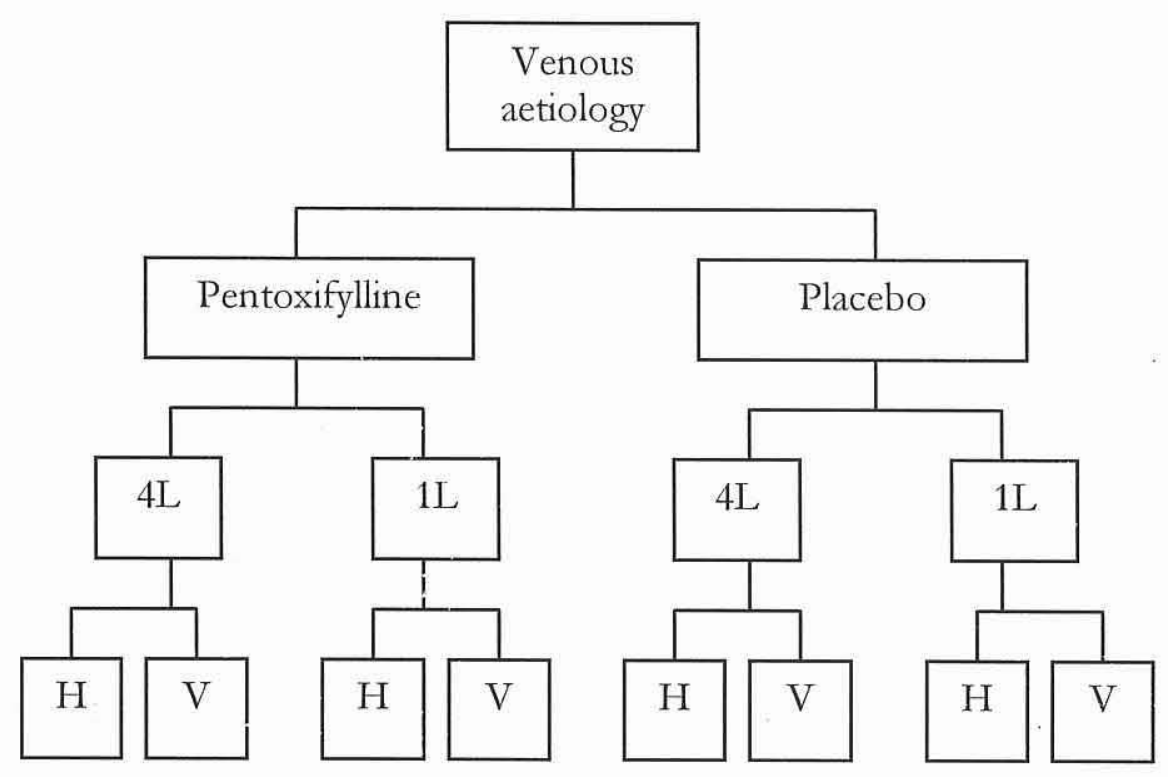

Figure 3: Treatments with factorial trial of Dale, et al. (1999): 4L, four layer; $1 \mathrm{~L}$, one layer; $H$, Hydrocolloid; $V$, Viscose.

clinical assessment, calculation of $\mathrm{ABI}$ and Doppler demonstration of venous pathology (sapheno-femoral and, or sapheno-popliteal reflux) in the same leg as the ulceration (Prescott, et al., 1998). Falanga, et al. (1999) relied upon clinical assessment (presence of hyperpigmentation, lipodermatosclerosis, varicose veins and medial location of the ulcer) and the presence of venous reflux as determined by continuous wave Doppler ultrasound. As indicated earlier Schürmann and Eberhardt (1986) did not report how their participants were determined to have venous ulceration, although some determination is implicit in the trial's title, as it referred to participants having varicose or post-thrombotic ulcers.

Four trials varied in the type of compression bandaging systems used. Schürmann and Eberhardt (1986) did not report the type of compression used. Both Colgan, et al. (1990) and Barbarino (1992) described the use of a two layer bandaging system, although the type of two layer system (or the brand) is not described. Colgan, et al. (1990) state that the application technique was standardised, but do not report how this was determined. Dale, et al. (1999) used two different bandaging systems balanced between the two arms: 97 participants used a four layer elastic bandage and the other 103 used a single layer inelastic system. Falanga, et al. (1999) used Unna boot to achieve compression; this consists of a zinc-impregnated bandage and a 
short-stretch elastic bandage. Standardisation of application of compression was not reported in either of these two trials. One trial indicated the dressings and bandages were changed at least weekly or as required (Dale, et al., 1999). None of the other four studies indicated the frequency at which dressings or bandages were changed.

Three trials (Colgan, et al., 1990; Dale, et al., 1999; Falanga, et al., 1999) were multicentre trials with treatment based in the community. Schürmann and Eberhardt (1986) did not report the setting. Barbarino (1992) was a single centre study and the participants received the majority of treatment within the community, but were treated in hospital for the first seven days after randomisation. During this period, the participants received either a twice-daily dose of pentoxifylline $200 \mathrm{mg}$ via the intravenous route or a matching placebo. This was in addition to pentoxifylline $1200 \mathrm{mg}$ in three divided doses via the oral route or a matching placebo. Barbarino (1992, p548) also tested each patient for "individualised responsiveness to the active treatment" and then underwent a washout period of two weeks duration prior to the study commencing. Schürmann and Eberhardt (1986), Colgan, et al. (1990), Dale, et al. (1999) and Falanga, et al. (1999) all compared pentoxifylline $1200 \mathrm{mg}$ daily in three divided doses (400mg) with a matching placebo. Additionally, Falanga, et al. (1999) compared pentoxifylline $2400 \mathrm{mg}$ daily in three divided doses $(800 \mathrm{mg}$ ) with a matching placebo.

The trials varied as to the investigators' treatment of participants with multiple ulcers. Colgan, et al. (1990) reported 18 patients in the treatment group and 24 in the control group had other ulcers, although it is not reported if this was on the same leg as the reference ulcer. The largest ulcer was used as the reference ulcer if it was within $2-15 \mathrm{~cm}$ in diameter, and the number of participants with healed reference ulcers within 24 weeks were reported. However, only 2 participants with multiple ulcers (one in each group) had an additional ulcer still present after the reference ulcer had healed. Dale, et al. (1999) and Falanga, et al. (1999) used one leg as the reference leg and did not categorise the participant as healed unless all ulcers on the reference leg were completely re-epitheliased within 24 weeks of treatment. Schürmann and Eberhardt (1986) and Barbarino (1992) reported the area of ulceration, but not the number of ulcers for each participant. The authors did not record how multiple ulcers (if any) were treated. 


\section{Methodological Quality of Included Studies}

Studies varied in quality and, or reporting of methods. All studies were described as randomised controlled trials, although randomisation strategies were reported in only two studies (Colgan, et al., 1990; Dale, et al., 1999) and allocation concealment in one (Dale, et al., 1999) Four trials were double-blind, although only Dale, et al. report how this was achieved (Prescott, et al, 1998). Schürmann and Eberhardt (1986) was described as a single-blind trial, although it is not reported whether the participants, investigators or assessors were blinded. None of the trials reported any unblinding, so it was assumed blinding was satisfactory. Inclusion criteria were reported in all but 1 trial (Schürmann \& Eberhardt, 1986), although this trial stipulated varicose and postthrombotic ulcers in the paper's title. Exclusion criteria were reported in this trial, although one other study did not do so (Colgan, et al., 1990). One trial did not report any data on baseline comparability (Schürmann \& Eberhardt, 1986), although the authors stated data was comparable, and baseline mean ulcer size was able to be calculated from outcome data (ulcer size at baseline and conclusion for each patient).

All studies reported objective data from which comparisons could be established; data from Falanga, et al. (1999) had to be extrapolated from a life analysis table. Two studies reported a priori sample size calculations and the use of intention to treat analysis (Dale, et al., 1999; Falanga, et al., 1999). Falanga, et al. (1999) used intention to treat analysis for all patients who had enrolled and for whom one follow-up visit was documented. This excluded 2 participants from the final analysis. Three other studies either had no withdrawals (Barbarino, 1992; Schürmann \& Eberhardt, 1986) or included the withdrawals in the analysis as treatment failures (Colgan, et al., 1990).

The included studies were assessed using following quality criteria and each study is reported below.

․ Inclusion and exclusion criteria

ㅁ A priori sample size calculation

$\square$ Method of randomisation

․ Allocation concealment

$\square$ Baseline comparability of groups

$\square$ Blinding

$\square \quad$ Appropriate outcome measures 
Schürmann and Eberhardt (1986): 24 participants in 2 arms; inclusion criteria not reported; exclusion criteria listed; a priori sample size calculation not reported; method of randomisation not reported; allocation concealment unclear; baseline comparability data not reported, although age, weight, height and ulcer history stated as being comparable; single-blind randomised controlled trial; appropriate outcome measures reported (ulcer size for each patient at baseline and trial completion); analysis by intention to treat unclear, but no patients withdrew.

Colgan, et al. (1990): 80 patients in 2 arms; inclusion criteria stated; exclusion criteria not listed; a priori sample size calculation not reported; randomisation by balanced blocks of eight in separate lists for each centre; allocation concealment unclear; groups comparable for age, sex, mean ulcer duration, mean ulcer size, resting anklebrachial index ( $\mathrm{ABI}$ ) and Basal Metabolic Index (BMI); double-blinding used; appropriate outcome measures reported (numbers of ulcers healed); analysis by intention to treat unclear, but withdrawals included in results as treatment failures.

Barbarino (1992): 12 patients in 2 arms; inclusion and exclusion criteria stated; a priori sample size calculation not reported; method of randomisation not reported; allocation concealment unclear; groups comparable for age, sex, mean duration of ulcer, mean ulcer size and resting ABI; double-blinding used; appropriate outcome measures reported (ulcer size for each patient at baseline and trial completion); analysis by intention to treat unclear, but no patients withdrew.

Dale, et al. (1999): 200 patients in 2 arms within a factorial trial; inclusion and exclusion criteria stated; a priori sample size calculation reported $(80 \%$ power to detect a $20 \%$ difference in treatment effects at $\mathrm{p}<0.05$ ) (Prescott, et al, 1998); randomisation by sequential sealed opaque envelopes; allocation concealment adequate; groups comparable for age, sex, median duration of ulcer and median ulcer size at baseline; double-blinding used; appropriate outcome measures reported (numbers of ulcers healed); analysis by intention to treat.

\footnotetext{
${ }^{1}$ Information regarding this trial was obtained from two sources; this source should be assumed to be the primary report unless indicated otherwise.
} 
Falanga, et al. (1999): 131 patients in 3 arms, 129 included in analysis; inclusion and exclusion criteria clearly stated; a priori sample size calculation reported; method of randomisation not reported; allocation concealment unclear; groups comparable for age, sex, mean duration of ulcer, mean resting $\mathrm{ABI}$ and numbers of ulcers per participant at baseline; double- blinding used; appropriate outcome measures reported (numbers of ulcers healed, median time to healing), but outcome measure reported using life-analysis table requiring results to be estimated from table; analysis by intention-to-treat.

\section{Comparisons of Studies}

The majority of studies reported either complete healing of the reference ulcer or all ulcers on the reference leg as the primary outcome, or provided individual data from which proportions healed could be calculated. In one study (Falanga, et al., 1999) proportions healed were extrapolated from a life-analysis table. The principal reviewer and one co-reviewer (JW) independently determined and agreed upon the estimate. Falanga, et al. (1999) also compared two different doses of pentoxifylline (1200mg and $2400 \mathrm{mg}$ daily) with the placebo. For the purposes of this analysis, the two treatment arms were added together; sensitivity analyses were conducted to test the impact of this. Falanga, et al. (1999) also excluded 2 participants from the analysis after randomisation despite stating analysis was by intention-to-treat. The report did not identify which group they were excluded from. In order to avoid any overestimation of treatment effect associated with this action, the excluded participants were included in the treatment as treatment failures, thus altering the denominator only. For the analysis of adverse effects, the 2 excluded participants were included as having suffered side effects, thus altering the numerator and the denominator.

Five studies (Schürmann \& Eberhardt, 1986; Colgan, et al., 1990; Barbarino, 1992; Dale, et al., 1999; Falanga, et al., 1999) compared pentoxifylline to placebo with compression as a standard therapy. All trials reported complete healing as a primary outcome. The results are presented graphically in the Peto tables. A fixed effects model was used to combine the 5 trials, the pooled relative risk (RR) was 1.30 (95\% CI 1.10-1.54) indicating a significantly greater likelihood of complete healing occurring if pentoxifylline is used in addition to compression bandaging (Figure 4, see 
below). The result was robust to combination using a random effects model, although the result just attained significance (RR 1.32, 95\%CI 1.01-1.71). However, the absence of significant heterogeneity (Chi-square 6.36, $\mathrm{df}=4, \mathrm{p}=0.17$ ) suggests combination by fixed effects model is appropriate

\begin{tabular}{|c|c|c|c|c|c|}
\hline Study & $\begin{array}{c}\text { Treatment } \\
\mathbf{n} / \mathbf{N}\end{array}$ & $\begin{array}{c}\text { Control } \\
\mathrm{n} / \mathrm{N}\end{array}$ & $\begin{array}{c}\text { RR } \\
\text { (95\% Cl Fixed) }\end{array}$ & $\underset{\substack{\text { Weight } \\
\%}}{ }$ & $\begin{array}{l}\text { RR } \\
\text { (95\% Cl Fixed) }\end{array}$ \\
\hline Barbarino 1992 & $4 / 6$ & $1 / 6$ & 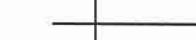 & $\rightarrow \quad 1.0$ & $4.00[0.61,26.12]$ \\
\hline Colgan 1990 & $23 / 38$ & $12 / 42$ & $\longrightarrow$ & 10.9 & $2.12[1.23,3.65]$ \\
\hline Dale 1999 & $65 / 101$ & $52 / 99$ & 불 & 50.2 & $1.23[0.97,1.55]$ \\
\hline Falanga 1999 & $61 / 86$ & $28 / 45$ & - & 35.1 & $1.14[0.87,1.49]$ \\
\hline Schürman 1986 & $2 / 12$ & $3 / 12$ & $\Gamma$ & 2.9 & $0.67[0.13,3.30]$ \\
\hline $\begin{array}{l}\text { Total( }(95 \% \mathrm{Cl}) \\
\text { Chi-square } 6.36 \text { (df=4) P: } 0.17\end{array}$ & $155 / 243$ & $96 / 204$ & $\phi$ & 100.0 & $1.30[1.10,1.54]$ \\
\hline & & & Fav & nt & \\
\hline
\end{tabular}

Figure 4: Comparison of pentoxifylline and compression versus placebo and compression (fixed effects model).

The comparison was not sensitive to exclusion of the $2400 \mathrm{mg}$ arm from Falanga, et al. (1999) (RR 1.31, 95\%CI 1.10-1.57; Figure 5) or the exclusion of Schürmann and Eberhardt (1986), the sole single-blind study (RR 1.32, 95\%CI 1.12-1.57; Figure 6).

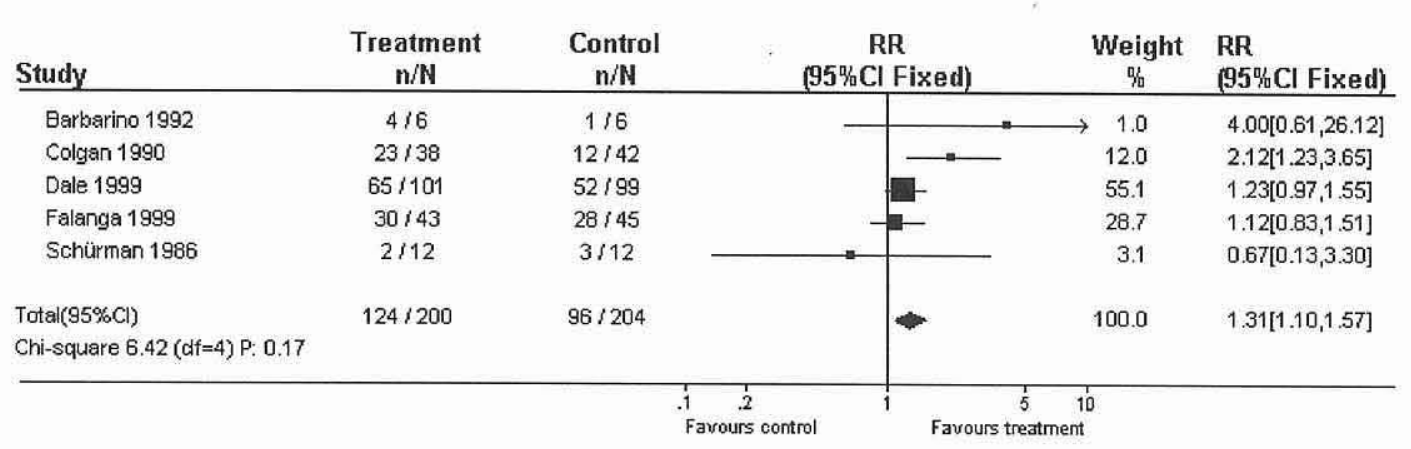

Figure 5: Sensitivity analysis - excluding 2400mg arm (fixed effects model).

\begin{tabular}{|c|c|c|c|c|c|}
\hline Study & $\begin{array}{c}\text { Treatment } \\
n / N\end{array}$ & $\begin{array}{c}\text { Control } \\
\mathrm{n} / \mathrm{N}\end{array}$ & $\begin{array}{c}\text { RR } \\
(95 \% \mathrm{Cl} \text { Fixed) }\end{array}$ & $\begin{array}{c}\text { Weight } \\
\%\end{array}$ & $\begin{array}{l}\text { RR } \\
\text { (95\%Cl Fixed) }\end{array}$ \\
\hline Barbarino 1992 & $4 / 6$ & $1 / 6$ & 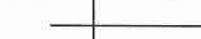 & 1.0 & $4.00[0.61,26.12]$ \\
\hline Colgan 1990 & $23 / 38$ & $12 / 42$ & $\longrightarrow$ & 11.2 & $2.12[1.23,3.65]$ \\
\hline Dale 1999 & $65 / 101$ & $52 / 99$ & $E$ & 51.7 & $1.23[0.97,1.55]$ \\
\hline Falanga 1999 & $61 / 86$ & $28 / 45$ & -5 & 36.2 & $1.14[0.87,1.49]$ \\
\hline $\begin{array}{l}\text { Total(95\%Cl) } \\
\text { Chi-square } 5.83(\mathrm{di}=3) \text { P: } 0.12\end{array}$ & $153 / 231$ & $93 / 192$ & $\Rightarrow$ & 100.0 & $1.32[1.12,1.57]$ \\
\hline & & & Farou & $\begin{array}{l}10 \\
10\end{array}$ & \\
\hline
\end{tabular}

Figure 6: Sensitivity analysis - double blind studies only (fixed effects model).

Three of the five trials reported treatment took place in a community or outpatient setting. Schürmann and Eberhardt (1986) did not report a setting and participants in 
Barbarino (1992) spent 7 days as hospital inpatients receiving both intravenous and oral treatment. The comparison was not sensitive to inclusion of only trials that reported outpatient treatment alone (RR 1.30, 95\%CI 1.09-1.54; Figure 7) or oral treatment only trials (RR 1.28, 95\%CI 1.08-1.51; Figure 8). Three of the trials reflected international prevalence studies with a greater ratio of female to male participants enrolled. Schürmann and Eberhardt (1986) did not identify the sex of

\begin{tabular}{|c|c|c|c|c|c|}
\hline Study & $\begin{array}{c}\text { Treatment } \\
\mathbf{n} / \mathbb{N}\end{array}$ & $\begin{array}{c}\text { Control } \\
\mathrm{n} / \mathrm{N}\end{array}$ & $\begin{array}{c}\mathrm{RR} \\
\text { (95\%Cl Fixed) }\end{array}$ & $\begin{array}{c}\text { Weight } \\
\%\end{array}$ & $\begin{array}{l}\text { RR } \\
\text { (95\%Cl Fixed) }\end{array}$ \\
\hline Colgan 1990 & $23 / 38$ & $12 / 42$ & $\because-$ & 11.3 & $2.12[1.23,3.65]$ \\
\hline Dale 1999 & $65 / 101$ & $52 / 99$ & - & 52.2 & $1.23[0.97,1.55]$ \\
\hline Falanga 1999 & $61 / 86$ & $28 / 45$ & - & 36.5 & $1.14[0.87,1.49]$ \\
\hline Total( $(95 \% \mathrm{Cl})$ & $149 / 225$ & $92 / 186$ & 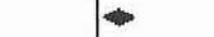 & 100.0 & $1.30[1.09,1.54]$ \\
\hline Chi-square 4.26 (df=2) P: 0.12 & & & & & \\
\hline
\end{tabular}

Figure 7: Sensitivity analysis - excluding trials not specifying an outpatient only setting (fixed effects model).

\begin{tabular}{|c|c|c|c|c|c|}
\hline Study & $\begin{array}{c}\text { Treatment } \\
\mathrm{n} / \mathrm{N}\end{array}$ & $\begin{array}{c}\text { Control } \\
\mathrm{n} / \mathrm{N}\end{array}$ & $\begin{array}{c}\text { RR } \\
\text { (95\%Cl Fixed) }\end{array}$ & $\begin{array}{c}\text { Weight } \\
y_{0}\end{array}$ & $\begin{array}{l}\text { RR } \\
\text { (95\% Cl Fixed) }\end{array}$ \\
\hline Colgan 1990 & $23 / 38$ & $12 / 42$ & $\longrightarrow$ & 11.0 & $2.12[1,23,3.65]$ \\
\hline Dale 1999 & $65 / 101$ & $52 / 99$ & - & 50.7 & $1.23[0.97,1.55]$ \\
\hline Falanga 1999 & $61 / 86$ & $28 / 45$ & - & 35.5 & $1.14[0.87,1.49]$ \\
\hline Schürman 1986 & $2 / 12$ & $3 / 12$ & & 2.8 & $0.67[0.13,3.30]$ \\
\hline $\begin{array}{l}\text { Totall(95\% } 6 \text { Cl) } \\
\text { Chi-square } 4.80 \text { (df=3) P: } 0.19\end{array}$ & $151 / 237$ & $95 / 198$ & - & 100.0 & $1.28[1.08,1.51]$ \\
\hline
\end{tabular}

Figure 8: Sensitivity analysis - excluding trials not speciffing oral treatment only (fixed effects model).

their participants and Falanga, et al. (1999) had a greater ratio of male to female participants despite being a 14 centre trial. The significance of the comparison was not sensitive to exclusion of this trial, although the precision was influenced (RR 1.39; 95\%CI 1.12-1.73; Figure 9).

\begin{tabular}{|c|c|c|c|c|c|}
\hline Study & $\begin{array}{c}\text { Treatment } \\
\mathbf{n} / \mathbf{N}\end{array}$ & $\begin{array}{c}\text { Control } \\
\mathrm{n} / \mathrm{N}\end{array}$ & $\begin{array}{c}\text { RR } \\
(95 \% \text { Cl Fixed) }\end{array}$ & $\begin{array}{c}\text { Weight } \\
\%\end{array}$ & $\begin{array}{l}\text { RR } \\
\text { (95\%CI Fixed) }\end{array}$ \\
\hline Barbarino 1992 & $4 / 6$ & $1 / 6$ & 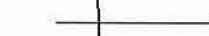 & 1.5 & $4.00[0.61,26.12]$ \\
\hline Colgen 1990 & $23 / 38$ & $12 / 42$ & $\longrightarrow$ & 16.8 & $2.12[1.23,3.65]$ \\
\hline Dale 1999 & $65 / 101$ & $52 / 99$ & 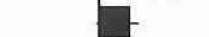 & 77.3 & $1.23[0.97,1.55]$ \\
\hline Schürman 1988 & $2 / 12$ & $3 / 12$ & & 4.4 & $0.67[0.13,3.30]$ \\
\hline $\begin{array}{l}\text { Total(95\%Cl) } \\
\text { Chi-square } 5.44 \text { (df=3) P: } 0.14\end{array}$ & $94 / 157$ & $68 / 159$ & - & 100.0 & $1.39[1,12,1.73]$ \\
\hline
\end{tabular}

Figure 9: Sensitivity analysis - exclusion of trials not specifying participants' sex or counter to trends in prevalence studies (fixed effects model). 
Three trials excluded participants with diabetes, a fourth did not specify whether diabetic participants were excluded (Schürmann \& Eberhardt, 1986), and a fifth (Colgan, et al., 1990) reported including diabetic participants and the group to which they were randomised in a letter regarding the trial (Colgan, 1990b). Exclusion of the trial which did not report exclusion of diabetics (Schürmann \& Eberhardt, 1986) and reallocation of three treatment successes as treatment failures in the pentoxifylline group in Colgan, et al. (1990) to test the worst case scenario had little impact on the summary relative risk (RR 1.29, 95\%CI 1.09-1.53; Figure 10).

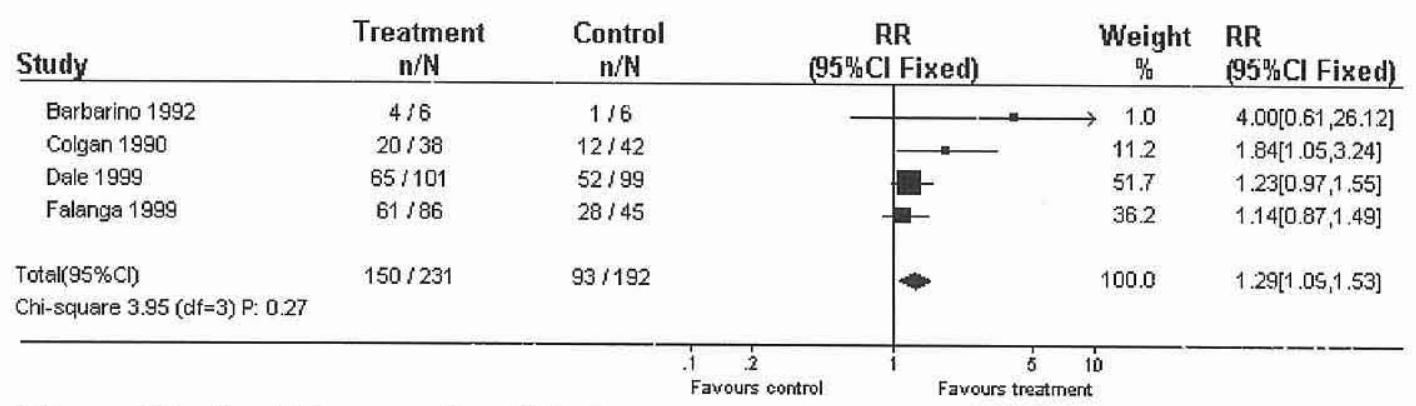

Figure 10: Sensitivity analysis - diabetic participants (fixed effects model).

Two trials (Barbarino, 1992; Schürmann \& Eberhardt, 1986) had a duration of 8-12 weeks and three, a duration of 24 weeks (Colgan, et al., 1990; Dale, et al., 1999; Falanga, et al., 1999). The summary odds ratio for trials of 8-12 weeks duration was not significant with a relative risk 0.71 (95\%CI $0.16-3.21)$.

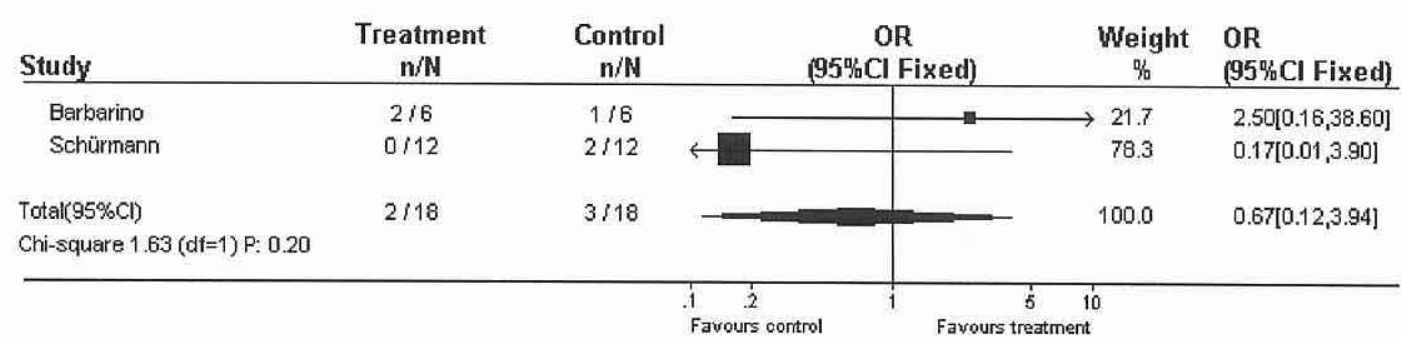

Figure 11: Comparison - trial duration 8-12 weeks (fixed effects model).

The greater numbers of participants were involved in the trials of 24 weeks duration. The summary relative risk for these trials was 1.30 (95\%CI 1.09-1.54; Figure 12). Heterogeneity was not significant and the exclusion of the $2400 \mathrm{mg}$ arm of Falanga et al (1999) also had little impact (RR 1.31, 95\%CI 1.09-1.56; Figure 13). 


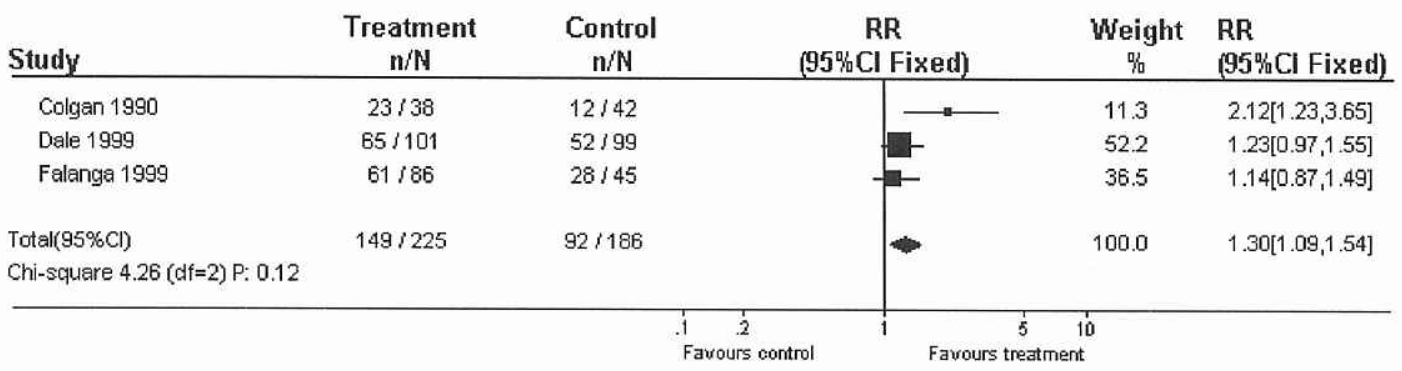

Figure 12: Comparison - trial duration 24 weeks (fixed effects model).

\begin{tabular}{|c|c|c|c|c|c|}
\hline Study & $\begin{array}{c}\text { Treatment } \\
\mathrm{n} / \mathrm{N}\end{array}$ & $\begin{array}{c}\text { Control } \\
\mathrm{n} / \mathrm{N}\end{array}$ & $\begin{array}{c}\text { RR } \\
(95 \% \mathrm{Cl} \text { Fixed) }\end{array}$ & $\begin{array}{c}\text { Weight } \\
\%\end{array}$ & $\begin{array}{l}\text { RR } \\
\text { (95\% Cl Flyed) }\end{array}$ \\
\hline Colgan 1990 & $23 / 38$ & $12 / 42$ & $\Longrightarrow$ & 12.5 & $2.12[1,23,3.65 !$ \\
\hline Dale 1999 & $65 / 101$ & $52 / 99$ & - & 57.5 & $1.23[0.97,1.55]$ \\
\hline Falanga 1999 & $30 / 43$ & $28 / 45$ & -5 & 30.0 & $1.12[0.83,1.51]$ \\
\hline $\begin{array}{l}\text { Total(95\%Cl) } \\
\text { Chi-square } 4.31 \text { (di=2) P: } 0.12\end{array}$ & $118 / 182$ & $92 / 186$ & 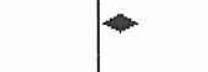 & 100.0 & $1.31[1.09,1.56]$ \\
\hline
\end{tabular}

Figure 13: Sensitivity analysis - Trial duration 24 weeks excluding $2400 \mathrm{mg}$ arm (fixed effects model).

The incidence of adverse effects was higher in the treatment group in 4 of the 5 trials (Barbarino, 1992; Colgan, et al., 1990; Dale, et al., 1999; Falanga, et al., 1999), although the summary relative risk was non-significant $(1.14,95 \%$ CI $0.79-1.66$ Figure 14). Heterogeneity was not significant and the summary results were not sensitive to the exclusion of the $2400 \mathrm{mg}$ arm in Falanga et al (1999) from the analysis (RR 1.05, 95\% CI 0.69-1.58). The most frequent adverse effects were gastro-intestinal (34 percent). In the two studies that reported reasons for withdrawal (Colgan, et al., 1990; Dale, et al., 1999), 7 of the 21 withdrawals (30 percent) were because of adverse effects.

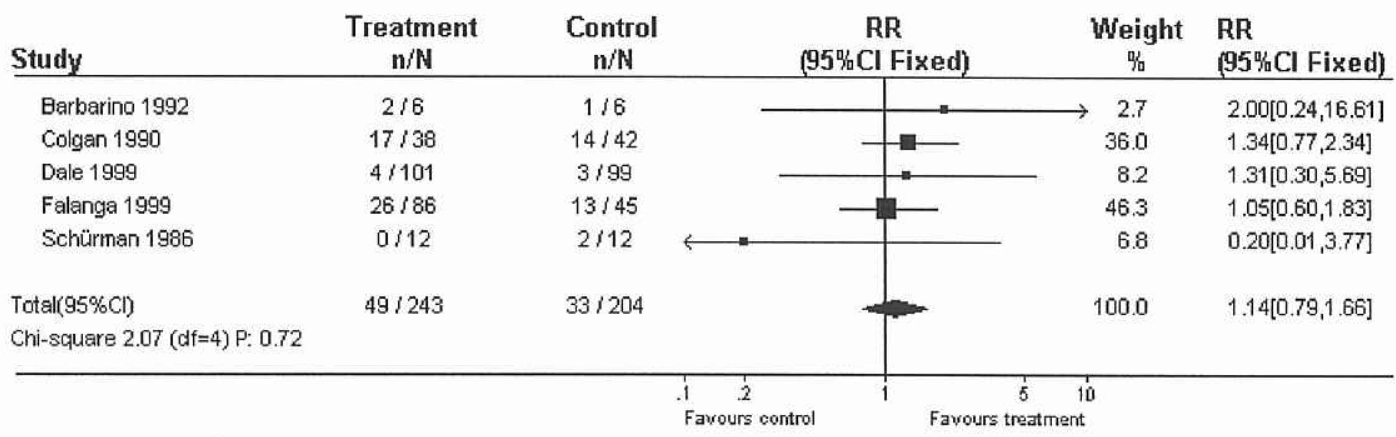

Figure 14: Comparison - adverse effects, pentoxifylline versus placebo (fixed effects model). 


\section{Chapter 5. DISCUSSION}

There is no gold standard test for venous ulceration. Diagnosis is made probabilistically by excluding other likely causes of ulceration, which can be complicated by the presence of co-morbidities. Baker, et al. (1991) noted that 5.5\% in sampled venous ulcers, the participants had diabetes, whilst $6.7 \%$ had rheumatoid arthritis, both diseases that are associated with leg ulceration. Nelzén, et al. (1994) noted a similar number $(8 \%)$ having diabetes in their study. Several studies in this meta-analysis attempted to limit the potential impact of mixed aetiology ulceration by excluding participants with co-morbidities such as diabetes (Barbarino, 1992; Dale et al., 1999; Falanga et al., 1999). However, Schürmann and Eberhardt (1986) did not specify co-morbidities as an exclusion criteria and Colgan, et al. (1990), whilst not specifying their exclusion criteria, later identified that 3 participants included in the treatment group had diabetes (Colgan 1990). Arguably, this makes the trial more valuable as it is likely to reflect what occurs in clinical practice, although it potentially dilutes inferences drawn about the casual relationship between pentoxifylline and ulcer healing when a single trial is considered.

The means of diagnosis differed between the studies. Schürmann and Eberhardt (1986) did not specify how the diagnosis of venous ulceration was made, although the study title makes it clear it was a trial involving participants with 'varicose' or post-thrombotic leg ulcers. The remaining four studies provided details of the diagnostic assessment, including the reporting of objective tests; three of the studies also specified a clinical history and examination as part of the diagnostic work up (Colgan, et al., 1990; Dale, et al., 1999; Falanga, et al., 1999). Given Barbarino (1992) identified the objective testing incorporated in their work up, it is improbable that the investigator did not also conduct a clinical history and examination.

The objective measures varied between the trials. Venous disease was determined using Doppler ultrasound measurement of venous reflux (Barbarino, 1992; Dale, et al., 1999; Falanga, et al., 1999) and the measurement of venous hypertension at the ankle (Barbarino, 1992). Doppler ultrasound measurement of the Ankle-Brachial Index ${ }^{1}$ to rule out arterial insufficiency was utilised, with the threshold of 0.8 usually reported (Barbarino, 1992; Colgan, et al., 1990; Dale, et al., 1999). Falanga et al

\footnotetext{
1 See page 43.
} 
(1999) reported a threshold of 0.5, although the mean ABI for both control and treatment groups was 1.1, indicating arterial insufficiency was not prevalent in their participants. Therefore, the reviewers believed that at the very least, the majority of participants had exclusively venous disease. Even if Schürmann and Eberhardt (1986), who did not report their inclusion criteria and the three participants from Colgan, et al. (1990) with diabetes were excluded from the analysis, 418 out of 447 met stringent inclusion criteria.

Compression significantly increases the proportion of healed ulcers in people with a venous aetiology. Multilayer bandaging (3 layer, 4 layer, Unna boot ${ }^{2}$ ) has been found to be more effective than single layer systems, although no specific type of multilayer system was favoured (Cullum, Fletcher, Nelson, \& Sheldon, 1999). Various multilayer systems were used by the included trials, Unna boot (Falanga, et al., 1999), and 2 layer systems (Barbarino, 1992; Colgan, et al., 1990). Dale, et al. (1999) used both single and 4 layer systems balanced in a factorial trial while Schürmann and Eberhardt (1986) did not specify what type of compression bandaging was used in their trial. Attempts by the reviewer to contact the investigators by mail and email were not successful. With the exception of Schürmann and Eberhardt (1986), the direction of effect ali favoured pentoxifylline suggesting a treatment effect that was independent of the type of compression bandaging used. The summary relative risk was $1.30(95 \%$ CI 1.10-1.54) and this was not sensitive to dose, type of blinding, route of drug, sex of the participants, or inclusion of the participants excluded by investigators after randomisation. The summary relative risk for adverse effects was not significant (RR 1.12, 95\% CI 0.79-1.66), although the incidence of adverse effects was higher in the pentoxifylline group. This result was not sensitive to dose. In the studies that described the adverse effects, the most frequent were gastrointestinal disturbances ( 34 percent). Of the two studies that did report reasons for withdrawals (Colgan, et al., 1990; Dale, et al., 1999), 7 of 14 cited the reason as side effects. This could suggest that many participants tolerated adverse effects, although those withdrawing because of adverse effects represented only 33 percent of participants in the treatment groups whom experienced a side effect. Additionally, one of the smaller studies reported no withdrawals (Barbarino, 1992), despite reporting incidence of adverse effects ( 2 out of 6 participants suffered transient nausea and headaches). Thus it would seem that the majority of those who experienced adverse

2 Unna boot is a multilayer bandage that consists of a zinc impregnated bandage and an elastic bandage i.e. Coban. 
effects tolerated them.

The majority of people with venous leg ulcers are managed in the community (Callam, Ruckley, Harper, \& Dale, 1985). One study (Barbarino, 1992) provided a combination of inpatient treatment for the first 7 days following entry into the trial and outpatient treatment for the following 60 days. Schürmann and Eberhardt (1986) did not report the study setting for their trial. The summary statistic was not sensitive to the exclusion of these trials, suggesting the treatment is effective in a community setting. As noted earlier, Colgan et al (1990) included 3 diabetic participants in the treatment groups without identifying their treatment outcomes. Schürmann and Eberhardt (1986) did not report that diabetic participants were included or excluded. The remaining trials excluded people with diabetes from participation. Exclusion of Schürmann and Eberhardt (1986), and the reallocation of 3 treatment successes in the pentoxifylline arm in Colgan et al (1990) to treatment failure tested the sensitivity of the summary statistic to the worst case scenario. The summary relative risk was not sensitive to this test (RR $1.29,95 \%$ CI 1.09-1.53).

The duration of treatment is an important clinical consideration. Two trials had a treatment maximum of 8-12 weeks (Barbarino, 1992; Schürmann \& Eberhardt, 1986). Combination of these trials produced a statistically non-significant result. The remaining 3 trials were of 24 weeks duration, with participants treated until complete healing or a maximum of 24 weeks. The summary statistic favoured pentoxifylline (RR 1.30, 95\% CI 1.09-1.54) and was not sensitive to the exclusion of the participants in Falanga, et al. (1999) who received the $2400 \mathrm{mg}$ dose. Examination of the life tables published in Colgan, et al. (1990), Dale, et al. (1999) and Falanga, et al. (1999) reveals a treatment effect does start to appear at 8-12 weeks; healing rates at 8 weeks were similar in Colgan, et al. (22\% for pentoxifylline versus $20 \%$ for controls) and Dale, et al. (30\% for pentoxifylline and $22 \%$ for controls). Only in Falanga, et al. (1999) did a divergence in healing rates appear earlier, with 8 week healing rates substantially different $(50 \%$ for the $2400 \mathrm{mg}$ dose group, $45 \%$ for the $1200 \mathrm{mg}$ dose group and $22 \%$ for the control group). The combined results for these trials, excluding the participants receiving the $2400 \mathrm{mg}$ dose of pentoxifylline, showed an absolute difference of 16.1 percent $^{3}$, giving a 'number needed to treat' (NNT) to

\footnotetext{
${ }^{3}$ The absolute difference is obtained by subtracting the desired outcome in the treatment group from the desired outcome in the control group.
} 
obtain one more healed ulcer when using pentoxifylline of 7 (95\% CI $4-16) .{ }^{4}$ Such a low NNT is more desirable than a high NNT, as it indicates only a small number of patients need to be treated to obtain one desired outcome. However, obtaining NNTs from meta-analysis can be misleading as just grouping the number of participants in the treatment arm and deriving a NNT ignores differences introduced by varying trial durations, doses, trends in incidence and the clinical setting (Smeeth, Haines, \& Ebrahim, 1999). It is also possible that the NNT may contravene the statistical assumptions underpinning combination by a fixed or random effects model. The fixed effects model assumes variations in trial samples are the result of random error, and the samples are all drawn from the same population. The random effects model assumes that the baseline characteristics reflect different populations, not random error. Thus combination of results derived from a random effects model contradicts the inherent assumption that the samples are drawn from different populations. Clarke and Oxman (1999) suggest that the NNT is an acceptable calculation when the duration of trials and dosages are the same, as is the case with the three trials from which this NNT was obtained.

Dosage is also an important clinical consideration. All but one trial utilised a $1200 \mathrm{mg}$ dose of pentoxifylline, this dose being that used in earlier trials with intermittent claudication. Falanga, et al. (1999) compared both $1200 \mathrm{mg}$ and $2400 \mathrm{mg}$ daily doses of pentoxifylline with the placebo. The rate of reported adverse effects was higher in the $2400 \mathrm{mg}$ group with 34.9 percent suffering adverse effects compared to 28.9 percent in the $1200 \mathrm{mg}$ group, although the withdrawal rate was similar in both groups. Virtually no difference between the two treatment groups was noted in the proportions healed at 24 weeks ( 74 percent for the $1200 \mathrm{mg}$ groups versus 72 percent for the $2400 \mathrm{mg}$ group). However, the investigators noted the median time to healing was lower in the $2400 \mathrm{mg}$ group at 71 days compared with the 83 days in the $1200 \mathrm{mg}$ groups. Median time for healing in the placebo group was 100 days. This suggests that although there appears to be no dose-dependent response in the numbers of people with healed ulcers, there may be a relationship between dose and time to healing. However, no other study reported either average or median time to healing and this suggested relationship would need further testing before such an inference could be stated.

\footnotetext{
${ }^{4}$ The NNT is calculated by obtaining the inverse of the absolute risk difference; the measure provides
} an estimate of the number of people who must be treated in order to obtain one beneficial outcome. 
The cost-effectiveness of treatment with pentoxifylline and compression has only been considered in association with one trial (Dale, et al., 1999). A full report of this is not yet available, although an abstract has been published in conference proceedings. Bosanquet, Franks, Brown, Straub, Harper and Ruckley (1995) reported that the total cost of ulcer care was greater in the placebo group $(£ 1,787)$ compared with pentoxifylline $1200 \mathrm{mg}(£ 1,524)$, concluding that pentoxifylline was more costeffective and healed more ulcers. However, this abstract presented no information on how the cost of treatment was assessed and thus, it is difficult to compare this result with even local cost environments.

Meta-analyses have become important because many trials are small and do not have sufficient power to detect small treatment effects. However, meta-analyses can mislead. LeLorier, Gregoire, Benhaddad, Lapierre \& Derderian (1997) compared the results of 12 large $(n>1000)$ randomised controlled trials (RCTs) with 19 metaanalyses published earlier on the same question. A total of 40 primary and secondary outcomes were measured and all large RCTs had to meet the a priori power calculation specified by the trial investigators. LeLorier, et al. (1997) found agreement between the two methods was only fair (kappa $=0.35$, 95\% CI 0.06-0.64), with the positive and negative predictive values of the meta-analyses 68 and 67 percent respectively. This would have led to adoption of ineffective treatments in 32 percent cases and rejection of effective treatments in 33 percent of cases. Although the difference in point estimates was significant in only 5 out of 40 comparisons, in each case a statistically significant effect was found by one method and no significant effect was found by the other method. Villar, Carroli and Belizán (1995) conducted a similar analysis with 30 meta-analyses in perinatal medicine. The results of the largest trial were removed from the meta-analyses, comparisons recalculated and compared to the result of the largest trial removed from each meta-analysis. The investigators found 24 meta-analyses correctly predicted the direction of effect for the largest trial, but in only 18 were both the same direction and statistical significance predicted. Publication of the Fourth International Study of Infarct Survival (ISIS-4) confirmed that meta-analyses can mislead. A meta-analysis of seven published trials $(n=1301)$ concluded that magnesium was an effective simple and safe treatment which reduces mortality following acute myocardial infarction (Yusuf, Koon, \& Woods, 1993). The ISIS-4 Collaborative Group (1995), with 58,050 participants, found no effect for magnesium sulphate. In examining why this might be so, Egger and Davey Smith (1995) found four possible causes. Firstly the meta-analysis had not included non- 
English language studies and the search strategy had not incorporated handsearching. Extending the search to include non-English language studies and using handsearching, Egger and Davey Smith (1995) found 5 small studies missed by the meta-analysis. Secondly, publication bias was evident in the distribution of the literature. This can be examined using funnel plots, in which effect sizes are plotted against sample size. If there is no publication bias, the plot should resemble an inverted symmetrical funnel, as the results of smaller studies are more scattered around the averaged effect size than those of the larger studies. ${ }^{5}$ A funnel plot of Yusuf, et al. (1993) revealed a highly asymmetrical plot, suggesting publication bias. Some of the trials in the meta-analysis were not analysed by intention-to-treat; when this was recalculated the significant differences became non-significant. Such biases in the included trials were the third potential reason for the difference between Yusuf, et al. (1993) and ISIS-4. The fourth possibility was that with so many metaanalyses now being conducted, a few will produce misleading results by chance. Should the concern that meta-analyses can mislead influence the interpretation of meta-analyses? LeLorier, et al. (1997) suggest it should. They believe that readers should go beyond simple point estimates when interpreting a meta-analysis. Instead the reader should investigate the included studies and look closely for consistency in the results. If the results of most included trials are on the same side of the forest plot, then the meta-analysis merits more confidence than if the results are distributed across the line of no effect. A similar point is made by Thompson and Pocock (1991). Examination of the forest plot for the five included trials here shows that four are on the same side of the line of no effect. This combined with the absence of significant heterogeneity should enhance confidence in the results of the metaanalysis.

A funnel plot of the studies reveals a symmetrical shape (Figure 16, see page 59) suggesting an absence of publication bias. However, the small number of studies and low power of this statistical technique at the best of times, means that this finding should be regarded with considerable caution (Clarke \& Oxman, 1999).

\section{Implications for Clinical Practice}

Although compression has improved rates of healing in people with venous ulcers, it

${ }^{5}$ The prevailing assumption is that larger studies are more precise and therefore likely to be closer to the average effect size. 


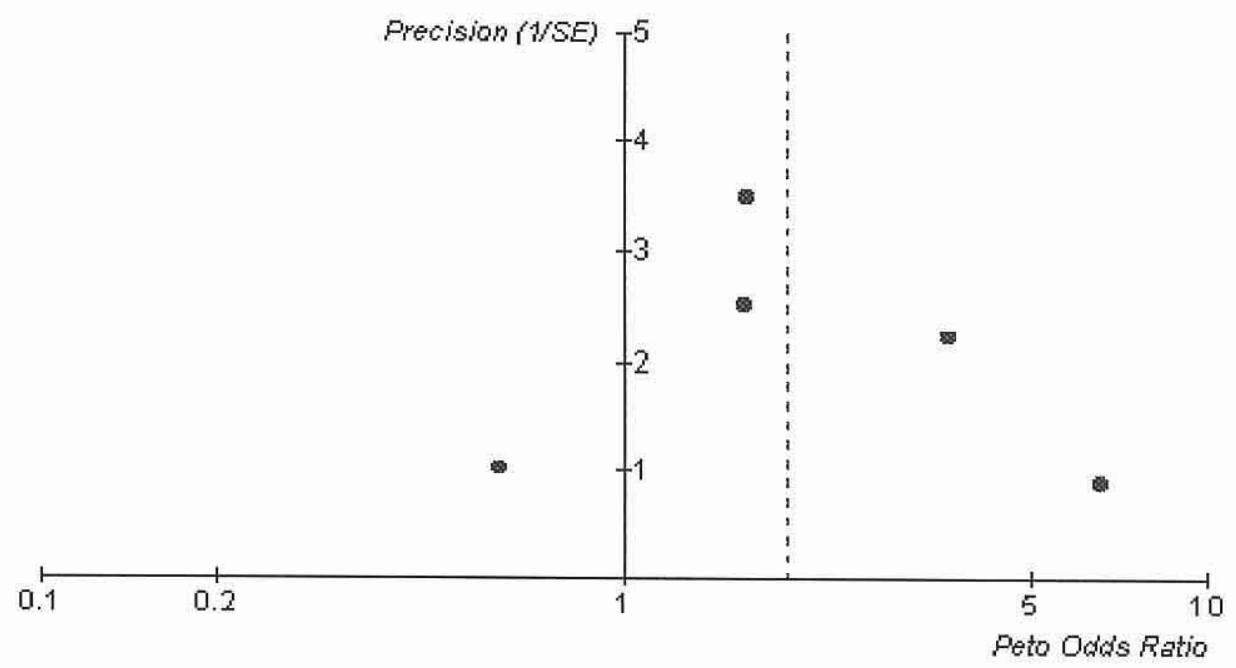

Figure 16: Funnel plot

is not effective in all instances. A recent cost-effectiveness study modelling the costs of various bandaging regimens and service delivery models drawn from a randomised controlled trial, assumed that $29 \%$ of ulcers would remain unhealed after 52 weeks treatment (Carr, Philips, \& Posnett, 1999). More importantly, the study assumed that no more people healed after week 24 when treated with compression. People whose ulcers do not heal remain a significant cost to the health system (Bosanquet, 1992); the overall costs must be greater when the impact on people's own lives is considered. Phillips, et al. (1994) reported that 76 percent of participants with an ulcer reported their financial situation was adversely affected by the presence of ulceration. Therefore, the addition of any adjunctive treatment, which improves rates of healing, especially in those with ulcers refractory to conventional treatment, is desirable. The two studies within this review that either stipulated therapy resistant ulcers as an inclusion criterion (Barbarino, 1992) or drew from a population of therapy resistant patients (Colgan, 1990b) found an effect in favour of pentoxifylline. Although statistical heterogeneity was non-significant, the clinical heterogeneity of these two trials was such that combination was unlikely to provide any useful information, particularly as the number of trials precluded sensitivity testing.

In summary, pentoxifylline is a useful adjunct to compression bandaging in the treatment of venous ulcers. The absence of any definitive evidence on the costeffectiveness or improved time to healing suggests that it should not be considered as routine treatment. However, patients with ulcers resistant to compression therapy may benefit from adjunctive pentoxifylline. This will be particularly important as the 
general population ages; the incidence and prevalence of ulceration increase with age (Baker, et al., 1991; Nelzén, et al., 1991). Minor gastrointestinal disturbances (nausea, vomiting and diarrhoea) are the most frequent adverse effect, but adverse effects seem likely to be tolerated by patients in the majority of cases. Although nurses are not able to prescribe pentoxifylline, knowledge of additional effective therapies is important in order to be able to inform patients as to treatment options, particularly when conventional therapy, such as compression, is ineffective.

\section{Implications for Research}

Methodologically the studies varied in quality and/or reporting of method. Although methodological quality and reporting quality can be confused, inadequate reporting of methods does not assist methodological assessment. All included studies were described as randomised controlled trials, although randomisation strategies were reported in only two studies (Colgan, et al., 1990; Dale, et al., 1999), and allocation concealment in one (Dale, et al., 1999). Four studies were described as double-blind comparisons (Colgan, et al., 1990; Barbarino, 1992; Dale, et al., 1999; Falanga, et al., 1999), although only one study described how this was achieved (Dale, et al., 1999). Blinding was described in a personal communication from the principal author in Falanga, et al. (1999). One trial was described as a single blind comparison, but again how this was achieved was not described (Schürmann \& Eberhardt, 1986). None of the five trials reported any unblinding of patients, so it was assumed blinding was satisfactory. Inclusion criteria were reported in all but one study (Schürmann \& Eberhardt, 1986), although this trial stipulated varicose and post-thrombotic ulcers in the paper's title. Exclusion criteria were reported in the trial and in all one other trials, bar one (Colgan, et al., 1990). One trial did not report any baseline data on the comparability of groups, although the authors reported the baseline data was comparable (Schürmann \& Eberhardt, 1986). However, mean ulcer size at baseline favoured the control group (pentoxifylline $5.4 \mathrm{~cm}^{2}$; control $2.5 \mathrm{~cm}^{2}$ ).

All studies reported objective outcome data from which comparisons could be established. Two studies (Dale, et al., 1999; Falanga, et al., 1999) used complete healing on single legs as the reference for the trial endpoint. Neither of these trials noted whether any participants had bilateral ulceration. Colgan, et al. (1990) used a single ulcer as the reference ulcer, where multiple ulcers were present. They noted that in all but 2 participants with multiple ulcers (one in each group), all other ulcers 
had healed by the time the reference ulcer had healed. Neither Schürmann and Eberhardt (1986), nor Barbarino (1992) identified whether their participants had multiple ulcers, although both recorded the total area of ulceration present in each participant. The use of single ulcers and legs as references for recording trial endpoints assumes that each ulcer heals independently of all others. This cannot be assumed when the therapy is systemic. Different bandaging pressures on different legs in participants with bilateral ulceration may confound healing. However, this reflects the clinical reality and for a truly accurate estimate of clinical effectiveness to emerge, all a participant's venous leg ulcers, regardless of site, should be healed for a positive outcome to be recorded.

Data from Schurmann and Eberhardt (1986) and Barbarino (1992) was calculated from the data at baseline and trial conclusion reported for each participant. Data from Falanga, et al. (1999) was extrapolated from a life analysis table; the coreviewers $(\mathrm{AJ} / \mathrm{JW})$ independently calculated and agreed upon this data. Two studies reported a priori sample size calculations and the use of intention to treat analysis (Dale, et al., 1999; Falanga, et al., 1999). Falanga, et al. (1999) used intention to treat analysis for all patients who had enrolled and for whom one follow-up visit was documented. This excluded 2 participants from the final analysis. The three remaining trials either had no withdrawals (Barbarino, 1992; Schürmann \& Eberhardt, 1986), or included the withdrawals in the analysis as treatment failures (Colgan, et al., 1990). The non-English language study (Schürmann \& Eberhardt, 1986) reported less methodological detail than did the English language studies. However, with exception to Dale, et al. (1999), attempts had to be made to contact all the studies' authors to gain further information. This was successful only in the case of Falanga, et al. (1999) and even then, not all the information requested was provided.

The important messages for future studies are:

1. The CONSORT (Consolidated Standards of Reporting Trials) statement should be used as a guideline for reporting (Begg, et al., 1996). A group consisting of editors, clinical epidemiologists, and statisticians wrote this statement in 1995, in an effort to improve the quality of written reports of randomised controlled trials. It has been adopted by journals such as JAMA and BMJ. 
2. Recruitment numbers should be based on a priori sample size calculation given that the likely treatment effect can now be inferred.

3. Treatment should include multi-layer compression bandaging as a standard therapy, unless the trial is assessing effectiveness in people unable to tolerate compression.

4. Where multiple ulcers exist, complete healing of all leg ulcers, even bilateral, should be the endpoint in the drug trial.

5. Short duration trials should be avoided, particularly as there is the suggestion that a treatment effect only begins to appear after 8-12 weeks of treatment.

6. Analysis should be by intention-to-treat of all participants following randomisation. Intention to treat analysis is generally taken as "including all patients, regardless of whether they actually satisfied the entry criteria, the treatment received and subsequent withdrawal or deviation from the protocol" (Hollis \& Campbell, 1999, p670).

7. Economic evaluations should be incorporated into trials in order to determine the cost-effectiveness of treatment. 


\section{Chapter 6. CRITICISM AND NON-RANDOM REFLECTIONS}

Meta-analysis has not been without its critics. Criticism has centred on the statistical combination of studies and it has been argued that meta-analysis combines 'apples and oranges', that garbage in leads to garbage out, and that the technique camouflages good scientific method with bad (Eysenck, 1978; Wolf, 1986; Wachter, 1988; Eysenck, 1994). These challenges have originated from opposition to the method in social sciences. When Smith and Glass (1977) conducted the first metaanalysis on psychotherapy outcomes, they included studies of any type of psychotherapy and all the literature they could identify that incorporated a control group, irrespective of methodologic quality. They identified 375 studies of 18 different treatments, with 40,000 treated and untreated participants, the treatment groups having received an average of 20 hours therapy by therapists with an average of 2.5 years experience. The participants themselves were a diverse group ranging from severe neurotics to people experiencing existential discomfort and a range of outcomes were reported.

The clinical heterogeneity of the studies in Smith and Glass's meta-analysis was considerable, leading to the 'apples and oranges' charge: the treatments were different, the study designs were different and the outcomes measured were different. Yet the authors were investigating the effectiveness of psychotherapy in general, not particular therapies, and hence the inclusive approach. The authors had been also inclusive in recognition that non-inclusion could introduce bias in the manner of traditional reviews.

The effectiveness of psychotherapy had troubled psychology and had been the focus of many investigations (Hunt, 1997), particularly following the publication of a review challenging its effectiveness (Eysenck, 1952). Eysenck compared the results of 19 studies of neurotic patients treated by psychotherapy with 2 samples of neurotics who received no treatment, and found that approximately two-thirds of neurotic patients would recover after 2 years, whether or not they were treated. A major criticism of this work was that it lumped together dissimilar patient groups and treatments. This was exactly the challenge that Eysenck, a major critic of metaanalysis made of Smith and Glass (1977) when he suggested meta-analysis combined 'apples and oranges' and thus was an exercise in 'mega-silliness' (Eysenck, 1978; 
Eysenck, 1994). Eysenck is also reported to have called meta-analysis an exercise in mega-imbecility (Hunt, 1997).

Central to Eysenck's criticism are issues of methodological rigour, principally concerns about heterogeneity, and the inclusion of methodologically poor studies. These concerns are not without due cause. Averaging studies which are dissimilar in demography, interventions, and outcomes, or, in other words, heterogenous, may produce misleading results and the inclusion of poor quality studies, even in a homogenous sample of studies, can lead to over-estimation of treatment effects as discussed previously (Egger \& Davey Smith, 1995; Moher, et al., 1998). This vulnerability has resulted in systematic review, with very specific questions, inclusion criteria, and methodologic assessment, being incorporated into meta-analysis (Gerbag \& Horwitz, 1988; Cook, Sackett, \& Spitzer, 1995; NHS Centre for Reviews and Dissemination, 1996). Caution should still be exercised when reading a meta-analysis, particularly one that includes a small number of studies. With small numbers, it cannot be certain that there has been an adequate sampling of the domain of potential participants, although this is offset by evidence of homogeneity in research questions, study designs and direction of effect. Meta-analysis requires methodologic vigilance, but should not be rejected as an exercise in 'mega-silliness'. The methodology has matured, addressed issues of rigour and now has a central place in research, being an essential element in effectiveness research. Eysenck too seems to have accepted that meta-analysis has a place "but only if attention is paid to the conditions that must be fulfilled in order to make the estimates applicable" (Eysenck, 1995, p35).

\section{Personal Reflections on Meta-analysis}

Systematic review and meta-analysis is a time consuming process, with much time committed to awaiting the results of literature searches, translating non-English language studies and seeking further information from trialists. Dickson and Entwistle (1996) reported spending 94 days searching the literature for a systematic review. Considerable amounts of time were also committed in this study to attempting communication with investigators in order to clarify methodological issues not addressed in reports, seeking suitably qualified translators and funding for translations. Study reports may not be available in New Zealand, or even indexed in MEDLINE $^{\circledR}$, which tends to run 6 to 12 weeks behind actual publication. Falanga, et 
al. (1999) was identified by the Cochrane Wound Group Co-ordinator after electronic searching had been completed. The journal in which it was published is not held in New Zealand, but is available in Australia, although an interloan request revealed that Australian libraries had not yet received this issue. The principal author's email address was identified by searching the Boston University's website ${ }^{1}$ and contact with the author duly produced a faxed imprint of the article. This was the only example of success using the Internet. Attempts to identify a contact for the authors of Schürmann and Eberhardt (1986) had very limited success. The employing institution of the corresponding author did have a website, but no list of staff contacts and it was clear from their response to an email query to the webmaster that communicating in English was not going to be successful in eliciting the necessary information. No other attempt to contact an author unknown to the reviewers was successful. It has been previously noted that a centralised register of trials would assist systematic reviewers. I would add that difficulties in locating authors would be greatly assisted by the maintenance of a database of trialists' current addresses, or where trialists are no longer available, identification of the location of the trial data and person(s) responsible for the data.

Translations are an absolute necessity for the conduct of a full systematic review, but locating translators with an understanding of health sciences is not simple, even for European languages. Locating a translator for non-European languages is even harder. Although the Cochrane Collaboration does try to co-ordinate translations through the Italian Cochrane Centre, attempts to have Brazilian, German and Italian studies translated through this centre were not successful. The suggested Portuguese translator did not respond to emails, the Italian translation did not arrive, despite being organised, and the German translation was completed only because I had learnt that personal contact was important. I utilised the Cochrane Co-ordinator of a local, but unrelated Review Group to organise the German translation, as she said she had been successful at this in the past. Attempts to use senior students through the Romance Languages Department at the local university were unsuccessful, with students stating they could not translate the articles after having reviewed them. Consequently, funding had to be obtained to translate the Brazilian and Italian studies by commercial translators, only to have them ruled out of selection as a consequence. Neither of these translators had experience in the health sector, and

${ }^{1}$ A previous article by the author identified his academic association. 
both required assistance, guidance and intuition from the principal reviewer, although this assistance was never acknowledged in the rates that we had to pay the translators.

\section{Nursing and Meta-analysis}

Pearcey (1995), in a survey of 398 nurses involved in education and clinical practice in a variety of settings, found that 93 percent were not satisfied with their research skills, despite 78 percent agreeing that the use of research findings could improve care or teaching. There was no significant difference in groups ordered by number of years trained and satisfaction with research skills, although staff on night duties did have significantly less satisfaction with their skills. The investigator noted that this was counter-intuitive to the perception that research is taught more extensively than ever before. The main self-perceived deficits were in locating, reading and evaluating research reports. In addition to little confidence in appraisal skills, most nurses also reported having very little time for reading research during their working day.

Meta-analyses are one more tool for addressing the need to drive evidence into practice. In aggregating studies addressing a particular question, quality assessing the studies against methodologic criteria that are known to bias results in controlled studies, and summarising the evidence, meta-analyses are a reliable and valid means for addressing the needs of clinical practice. Nursing has used meta-analysis from the early 1980s, although nursing's adoption of the methodology has not been as fast or extensive as in medicine. The first meta-analysis conducted by a nurse examined the effects of a behavioural intervention on weight loss in obese patients and was indexed in 1982 (O'Flynn, 1982a). The first published meta-analysis was indexed in 1983 (Devine \& Cook, 1983). Early meta-analyses in nursing drew heavily on psychology to examine the effects of behavioural therapy (O'Flynn, 1982a), psychoeducational interventions on hospital length of stay (Devine \& Cook, 1983), and preoperative instruction (Hathaway, 1986).

Droogan and Cullum (1998) explored the extent to which there existed systematic reviews of nursing effectiveness ${ }^{2}$, as part of the National Health Service Research and Development programme. Using electronic searching of computerised databases and

\footnotetext{
2 The term is used synonymously with meta-analysis by these authors.
} 
handsearching up to 1994, they identified a total of 36 papers, which specified either a question, a search strategy or reported the means of synthesis. Nineteen reviews met all 3 criteria, with the majority of these being published after 1990 . Seven of the 19 addressed patient education, perhaps reflecting the continuing influence of psychology on the use of meta-analysis in nursing. In 1982, O'Flynn (1982b, p316) had noted that meta-analysis was "an extremely efficient approach to the integration of studies and when appropriately used will expedite nursing's future endeavors [sic]." As evidenced by Droogan and Cullum's (1998) search, few nurses had taken to the methodology and the promise O'Flynn had noted, leading Droogan and Cullum to comment that there are

many important topics notewortby by their absence, with a paucity of reviews relating to the organisation of care...

(ibid., p18)

Textbooks on research methodologies in nursing have been slow to alert nurses to either the place of meta-analysis or the role of systematic review in research, even very recent publications. Examination of nine texts published between 1986 and 1999 (Hogstel \& Sayner, 1986; Burns \& Grove, 1987; Seaman, 1987; Wilson, 1989; Mateo \& Kirchhoff, 1991; Burnard \& Morrison, 1994; Robertson, 1994; LoBiondoWood \& Haber, 1998; Polit \& Hungler, 1999) found only four mentioned metaanalysis, and then with limited discussion and no criteria for critiquing the methodology (Burns \& Grove, 1987; Wilson, 1989; LoBiondo-Wood \& Haber, 1998; Polit \& Hungler, 1999). No text mentioned systematic review. All the texts discussed the purposes of literature review, but rarely in terms of research synthesis. More commonly literature reviews were discussed as a component of preparing a research report. None of the texts that discussed meta-analysis did so with reference to it as an advance on narrative reviews. Efforts such as the Cochrane Collaboration, the Joanna Briggs Institute of Evidence Based Nursing, and the McMaster Nursing Effectiveness, Utilisation and Outcomes Research Unit are starting to encourage the conduct and dissemination of meta-analyses relevant to nursing practice. However, it is clear that both the number of systematic reviews and the number of nurses involved in their conduct needs to increase. Health care is delivered by multidisciplinary teams and systematic reviews should reflect this where appropriate (Droogan \& Cullum, 1998). 


\section{Chapter 7. CONCLUSION}

The project has used meta-analysis to investigate the effect of pentoxifylline on venous ulcer healing when used as an adjunct to compression therapy. Ten randomised controlled trials were identified, using a strategy that searched databases created from frequent and extensive electronic searching and handsearching of relevant journals, conference proceedings and other grey literature. Five studies meet a priori specified inclusion criteria, and were aggregated utilising computer software distributed for the purpose by the Cochrane Collaboration. The trials did not exhibit any significant clinical or statistical heterogeneity and were combined to produce a summary statistic. The relative risk favoured pentoxifylline as an adjunct to compression bandaging, and this was robust to sensitivity analyses. Gastrointestinal disturbances are the most frequent adverse effect, but adverse effects appear to be tolerated by patients in the majority of cases.

The absence of any definitive evidence on the cost-effectiveness or improved time to healing suggests that it should not be considered at routine treatment. However, patients with ulcers resistant to compression therapy may benefit from adjunctive pentoxifylline. This will be particularly important as the general population ages; the incidence and prevalence of venous ulceration increase with age. Although nurses are not able to prescribe pentoxifylline, knowledge of additional effective therapies is important in order to be able to inform patients as to treatment options, particularly when conventional therapy, such as compression, is ineffective. The implications of this project for future research were considered and recommendations for the conduct of research and presentation of research reports results were made, to assist with future meta-analyses.

Nursing has yet to take up the early promise of meta-analysis, although a few nurses have conducted meta-analyses in areas of importance to nursing. Meta-analyses prepared by organisations such as the Cochrane Collaboration, will also inform and be of value to nursing practice. However, the requirement that valid meta-analyses incorporate as many studies as possible, irrespective of language, creates difficulties for their conduct, unless researchers are adequately supported to obtain translations. Such support could be improved, and would be greatly assisted by the maintenance 
of an international register of trialists with current contact addresses for occasions when clarification or further information is needed.

A number of fertile areas for further investigation have arisen through this metaanalysis. They include:

1. Further work to determine the cost-effectiveness of pentoxifylline and compression bandaging.

2. Further work to determine whether pentoxifylline is more effective on refractory venous ulcers.

3. Further work to determine whether time to healing is dose dependent. 


\section{EPILOGUE. Personal Reflections on Nursing Research}

There can be little question that systematic review and meta-analysis are of pragmatic use to nursing. But one of the many interesting questions raised by this project has been what is nursing research and could this project be considered such? Certainly the principal reviewer is a nurse by profession, but definition by this route only leads one to the misconception that research becomes nursing if conducted by nurses. The fallibility of this definition becomes obvious when it is considered in terms of logic statements: $\mathrm{A}$ is a nurse; $\mathrm{A}$ is conducting research; therefore, $\mathrm{A}$ is conducting nursing research. Yet a nurse conducting research in physical anthropology is unlikely to be conducting nursing research. Similarly, the use of particular methodologies does not make research nursing research. Nursing, in common with all the sciences and social sciences, employs a variety of methodologies developed by other disciplines, with the choice being determined by the type of phenomenon being investigated. For instance, causal relationships are best investigated using the randomised controlled trial, a methodology commonly associated with medicine, but which was developed for agricultural research.

This leaves only the content or context of the research as being definitive. The content of this project is the investigation of a drug therapy, a modality normally associated with the practice of medicine, not nursing. Burns and Grove (1987) have noted that knowledge can be drawn from other disciplines and integrated into the practice of nursing; where such transdisciplinary sharing takes place "it is difficult to know where the boundaries exist between nursing's knowledge base and those of other disciplines" (ibid. p21). In this project the key issue has been can a drug therapy assist in the healing of venous ulcers? Conventional treatments do not assist all people with leg ulcers and thus the use of pentoxifylline may benefit those who do not respond to conventional therapies alone. Nurses in the community are responsible for much of ulcer care (Callam, et al., 1985) and thus must be aware of other options where conventional therapies fail in order to adequately inform patients of what may be possible. This will be emphasised in New Zealand if prescribing rights are given to certificated nurses involved in aged care, as the incidence and prevalence of ulcers increases with age. The content of this project can be said to be of value to nursing, meeting McGowen's definition of nursing research, which is 
research into the process of delivery of care, and the clinical problems that are encountered in nursing practice

(McGowen, 1994, p1).

However, with nursing being at the nexus of patient care, it encounters many clinical problems that have been addressed by other disciplines, of which this project is but one example. Research into problems encountered by practitioners of nursing does not make the research nursing research. Thus, perhaps it is the context that is important. McGowen (1994) noted that unless the research addresses everyday issues associated with the practice of nursing, it cannot be considered nursing research. Yet. can investigation into the accuracy of digital thermometers be considered nursing research, even if conducted by nurses, in a nursing context? Accuracy of temperature readings is as important to general practitioners and parents as it is to the nurse. Burns and Grove (1987) attempt to differentiate the contextual nature of nursing research by suggesting differences in worldviews characterise disciplinary research. This assumes that all nursing researchers share the same worldview and that researchers in other disciplines cannot access or participate in that worldview. Furthermore, such an assertion relegates the individual researcher to something of a predetermined position, a channel through which the 'nursing' truth emerges. Certainly, paradigms can dominate a profession, and such paradigms may be expressed in the type of research generally conducted by that profession, but such pluralistic apartheid as expressed by Burns and Grove does not seem at home with the many interpretivist and constructivist approaches, where the investigator, the participants and the nature of their individual relationships define the outcome. To argue that a part is greater than and characterises the whole undermines the nature and value of such approaches.

If it is so difficult to define nursing research, does it exist? Research in nursing is vibrant, but this verb phrase does not carry the meaning as the noun phrase 'nursing research'. The transdisciplinary nature of knowledge seems to make the title nursing research as presumptuous as the title medical research. Where health work is concerned, with disciplinary boundaries becoming increasingly vague, it would seem health research and research in nursing practice is possible, but that nursing research as a pure disciplinary project is not. 


\section{Appendix 1: Contact Addresses For Co-Reviewers}

Dr Bruce Arroll MBChB PhD FRNZCGP FAFPHM

Associate Professor

Department of General Practice

Faculty of Medicine and Health Sciences

University of Auckland

Private Bag 92019

Auckland

Dr Jill Waters MBChB FRACP

Geriatrician

A+ Links

Auckland Hospital

Private Bag 92024

Auckland 


\title{
Appendix 2: Cochrane Collaboration Wound Review Group Search Strategy for Specialised Register of Trials (Central)
}

The editorial team of the Cochrane Wound Group assembles, maintains and administers a specialised register of controlled clinical trials at the editorial base. All trials in which allocation to an intervention has been described as random, or quasirandom are included in the register. Furthermore, all trials which are potentially random (i.e. the method of allocation is not stated as random but the trial design suggest that it may be so) are included. Thus potentially useful trials are not automatically excluded from the register.

\section{Electronic database searches:}

MEDLINE has been searched for randomised controlled trials (RCTs) and controlled clinical trials (CCTs) from 1966 to December 1997 using a mixture of free text terms and MeSH headings. From January 1998 CENTRAL is searched using a simplified search strategy including the following terms:

\author{
WOUND INFECTION \\ PILONIDAL CYST \\ WOUNDS AND INJURIES \\ WOUND HEALING \\ LEG ULCER \\ VARICOSE ULCER \\ SKIN ULCER \\ DECUBITUS
}

MEDLINE (Ovid version) 1966-1997

001 Decubitus ulcer/ or foot ulcer/

002 Leg ulcer/ or varicose ulcer/

003 Pilonidal cyst/

004 Skin ulcer/

005 Diabetic foot/

006 ((plantar or diabetic or heel or venous or stasis or arterial) adj ulcer $\$$ ).tw

007 ((decubitus or foot or diabetic or ischaemic or pressure) adj ulcer $\$$ ).tw

008 ((pressure or bed) adj sore $\$$ ).tw

009 ((pilonidal adj cyst) or (pilonidal adj sinus) or bedsore\$).tw

010 ((diabetic adj foot) or (cavity adj wound)).tw

011 ((varicose or leg or skin) adj ulcer\$).tw

012 ((decubitus or (chronic adj wound\$)).tw

013 ((sinus adj wound\$) or (cavity adj wound\$)).tw

014 or $/ 1-13$

015 debridement/ or biological dressings/ or bandages/

016 occlusive dressings/ or clothing/ or wound healing/

017 antibiotics/ or growth subsatnces/ or platelet derived growth factor/

018 fibroblast growth factor/ or electrical stimulation therapy.ti, ab, sh

019 lasers/ or nutrition/ or surgery/ or surgery, plastic/

020 surgical flaps/ or skin transplantations/ or homeopathy/ or homeopathic/

021 acupuncture therapy/ or acupuncture/ or alternative medicine/

022 massage/ or iloprost/ or alginates/

023 zinc/ or zinc oxide/ or ointments/ or anti-infective agents / 
024 dermatologic agents/ or colloids/ or cushions/ or wheelchairs/

025 beds/ or wound dressings/

026 (debridement or dressing $\$$ or compress $\$$ or cream $\$$ or (growth adj factor $\$$ )).tw

027 (pressure-relie $\$$ or (recombinant adj protein $\$$ ) or bandag $\$$ or stocking $\$$ ).tw

028 (antibiotic $\$$ or (electric adj therapy) or laser $\$$ or nutrition $\$$ or surg $\$$ ).tw

029 (homeopath $\$$ or acupunture or massage or reflexology or ultrasound).tw

030 (iloprost or alginate $\$$ or zinc or paste $\$$ or ointment $\$$ or hydrocolloid $\$$ ).tw

031 ((compression adj therapy) or (compression adj bandag\$) or wrap\$).tw

032 (bed $\$$ or matress $\$$ or wheelchair $\$$ or (wheel adj chair) or cushion $\$$ ).tw

033 ((wound adj dressing $\$$ ) or vitamin $\$$ or bind $\$$ or gauze $\$$ or heals or healing).tw

034 (diet or lotion $\$$ or infect\$ or reduc\$ or (wound adj healing)).tw

035 (treat $\$$ or prevent $\$$ or epidemiol $\$$ or aetiol $\$$ or etiol $\$$ or therap $\$$ or prevalence or incidence).tw

036 or $/ 15-35$

03714 and 36

038 random allocation/ or randomized controlled trials/

039 controlled clinical trials/ or clinical trials phase I/ or clinical trials phase II/ clinical trials phase III/ or clinical trials phase IV/

040 clinical trials overviews/

041 single-blind method/ or double blind method/

042 publication bias/ or review/ or review, academic/

043 review tutorial/ or meta-analysis/ or systematic review/

044 ((random $\$$ adj controlled adj trial\$) or (prospective adj random $\$$ ).tw

045 ((random adj allocation) or random $\$$ or (clinical adj trial\$) or control\$)).tw

046 ((standard adj treatment) or compar $\$$ or single-blind $\$$ or double-blind $\$$ ).tw

047 (blind $\$$ or placebo $\$$ or systematic $\$$ or (systematic adj review).tw

048 (randomized controlled trial or clinical trial).pt or comparative study.sh

049 or $/ 38-48$

05037 and 49

051 limit 50 to human

052 burns/ or wounds, gunshot/ or corneal ucler/ or exp dentistry/

053 peptic ulcer/ or duodenal ulcer/ or stomach ulcer/

054 ((peptic adj ulcer) or (duodenal adj ulcer) or traum\$).tw

055 ((aortocaval adj fistula) or (arteriovenous adj fistula)).tw

056 (bite adj wound\$).tw

057 or/ $52-56$

05851 not 57

EMBASE (Ovid version) 1980-1997

CINAHL (Ovid version) 1980-1997

001 Pressure ulcer/ or foot ulcer/ or leg ulcer/ or skin ulcer/

002 Diabetic foot/ or diabetic neuropathies/

003 Diabetic angiopathies/ or diabetes mellitus.co

004 Pilonidal cyst/ or surgical wound infection/

005 ((plantar or diabetic or heel or venous or stasis or arterial) adj ucler $\$$ ).tw

006 ((decubitus or foot or diabetic or ischaemic or pressure) adj ulcer\$).tw

007 ((pressure or bed) adj sore $\$$ ).tw

008 ((pilonidal adj cyst) or (cavity adj wound)).tw

009 ((diabetic adj foot) or (cavity adj wound).tw

010 ((varicose or leg or skin) adj ucler\$).tw

011 (decubitus or (chronic adj wound\$)).tw

012 ((sinus adj wound\$) or cavity adj wound\$)).tw 
013 or $/ 1-12$

014 debridement/ or biological dressings/ or occlusive dressings/

015 (bandages.ti,sh,ab, it and "Bandages and Dressings"/) or

016 compression garments/ or antibiotics/

017 electric stimulation/ or laser surgery/ or lasers.th/ nutrition care (Saba HHCC) / or diet therapy/ or nutritive therapy (Iowa NIC)/

018 surgery, reconstructive/ or surgery, plastic/ or surgical flaps/

019 surgical stapling/ or skin tranplantation/ or alternative therapies/

020 acupuncture/ or massage/ or zinc/ or ointments /

021 antiinfective agents, local/ antibiotics / or dermatologic agents /

022 dermatology nursing/ or colloids/ or beds and mattresses /

023 flotation beds/ or wheelchairs/ or positioning: wheelchair/ or positioning: therapy/

024 patient positioning/ or positioning/ or wound care/ or wound healing/

025 (debridement or dressing $\$$ or compress $\$$ or cream $\$$ ).tw

026 ((growth adj factor $\$$ ) or pressure relie $\$$ or (recombinant adj protein $\$$ ) or bandag $\$$.tw

027 (stocking $\$$ or antibiotic $\$$ or (electric adj therapy) or laser $\$$ or nutrition $\$$ or $\operatorname{surg} \$) . t w$

028 (iloprost or alginate $\$$ or zinc or paste $\$$ or ointment $\$$ or hydrocolloid $\$$ ).tw

029 ((compression adj therapy) or (compression adj bandag $\$$ ) or wrap $\$$ ).tw

030 (bed $\$$ or mattress $\$$ or wheelchair $\$$ or (wheel adj chair) or cushion $\$$.tw

031 ((wound adj dressing $\$$ ) or vitamin $\$$ or bind $\$$ or gauze $\$$ or heals or healing).tw

032 (diet or lotion $\$$ or infect $\$$ or reduc $\$$ or etiol $\$$ or (wound adj healing)).tw

033 (treat $\$$ or prevent $\$$ or epidemiol $\$$ or therap $\$$ or prevalence or incidence).tw

034 "Bandages and Dressings"/ or skin transplantation/ or homepathy/ or ointments/ or "bed and mattresses"

035 or $/ 14-34$

03613 and 35

037 clinical trials/ or single-blind studies/ or double-blind studies/

038 control group/ or placebos/ or meta-analysis/

039 ((random $\$$ adj clinical adj trial\$) or (prospective adj random\$)).tw

040 ((random adj allocation) or random $\$$ or controlled clinical trial $\$$ or control).tw

041 (comparison group $\$$ or (standard adj treatment) or compar\$).tw

042 (single-blind $\$$ or (single adj blind) or double-blind or (double adj blind)).tw

043 (blind $\$$ or placebo $\$$ or systematic or (systematic adj review)).tw

044 (meta analysis or meta-analysis).tw or (trials or trial or prospective).tw

045 (clinical trials).sh or (comparative studies).sh

046 or/ $37-45$

04736 and 46

048 burns/ or wounds, gunshot/ or corneal ulcer/ or exp dentistry/

049 peptic ulcer/ or duodenal ulcer/

050 ((peptic adj ulcer) or (duodenal adj ulcer) or trauma).tw

051 ((burn\$ or (gunshot adj wound\$) or (corneal adj ulcer) or dentist\$ or (bite adj wound\$)).tw

052 or $/ 48-51$

05347 not 52

The following databases have also been searched from the earliest available date:

ISI Science Citation Index (on BIDS)

BIOSIS (on Silver Platter)

British Diabetic Association Database 
CISCOM (Complementary Medicine Database of the RCCM)

Conference Proceedings (on BIDS)

Database of Abstracts of Reviews of Effectiveness (DARE)

Dissertation Abstracts

Royal College of Nursing Database

\section{Journal handsearches}

Wound care specialty journals have been handsearched as follows:

CARE - Science and Practice (later Journal of Tissue Viability) 1979-1990

Journal of Tissue Viability 1991-present

Decubitus 1987-1993

Journal of Wound Care 1991-present

Phlebology 1986-1995

\section{Conference Proceedings}

Proceedings of the 1st European Conference on Advances in Wound Management, 1991

Proceedings of the 2nd European Conference on Advances in Wound Management, 1992

Proceedings of the 3rd European Conference on Advances in Wound Management, 1993

Proceedings of the 4th European Conference on Advances in Wound Management, 1994

Proceedings of the 5th European Conference on Advances in Wound Management, 1995

Proceedings of the 6th European Conference on Advances in Wound Management, 1996

Proceedings of the 7th European Conference on Advances in Wound Management, 1997

3rd Annual Symposium on Advanced Wound Care, 1990

4th Annual Symposium on Advanced Wound Care, 1991

5th Annual Symposium on Advanced Wound Care, 1992

1996 Symposium on Advanced Wound Care \& Medical Research Forum on Wound Repair

Proceedings of: Going into the '90s: The Pharmacist and Wound Care,1992

Proceedings of the Second Joint British/Swedish Angiology Meeting,1991

Symposium on Venous Leg Ulcers, 1985

$3^{\text {rd }}$ Annual Symposium on Advanced Wound Care, 1990

$4^{\text {th }}$ Annual Symposium on Advanced Wound Care, 1991

$5^{\text {th }}$ Annual Symposium on Advanced Wound Care, 1992

Symposium on Venous Leg Ulcers, 1985

$2^{\text {nd }}$ Joint British/Swedish Angiology Meeting, 1991

Going into the '90s: The Pharmacist and Wound Care, 1992

1996 Symposium on Advanced Wound Care \& Medical Research Forum on Wound Repair 


\section{Unpublished Studies}

Several databases have been searched (up to December 1997) to attempt to identify unpublished studies. These include:

CRIB (Current Research in Britain)

DHSS Database

SIGLE (Systematic Index of Grey Literature in English)

HealthStar

UK National Research Register

Smith \& Nephew Wound Care Database

Amed

Experts in wound care and pharmaceutical companies were contacted to enquite about unpublished, ongoing and recently published trials. Citations within obtained reviews and papers were scrutinised to identify additional studies. 


\section{Appendix 3: Cochrane Collaboration Peripheral Vascular Diseases Review Group Search Strategy for Specialised Register of Trials (Central)}

The editorial team of the Cochrane Peripheral Vascular Diseases Group assembles, maintains and administers a specialised register of controlled clinical trials at the editorial base. The register presently contains 2754 references to relevant trials. Before a reference is entered onto the database, a photocopy is obtained, and codes assigned for characteristics of the trial such as the method of randomisation, disease being treated, and type of intervention. An additional database contains a further 1500 uncoded references.

All trials in which allocation to an intervention has been described as random, or quasi-random are included in the register. Furthermore, all trials which are potentially random (i.e. the method of allocation is not stated as random but the trial design suggest that it may be so) are included. Thus potentially useful trials are not automatically excluded from the register.

\section{Electronic Database Searches}

MEDLINE (OVID version) 1966 to 1999 (May)

MEDLINE has been systematically searched using the following strategies (the first part developed by Carol Lefebvre):

a) Identifying possible randomised controlled trials

001 randomised controlled trial.pt.

002 controlled clinical trial.pt.

003 randomized controlled trials/

004 random allocation/

005 double-blind method/

006 single-blind method/

007 exp evaluation studies/

008 exp clinical trials/

009 clinical trial.pt.

010 exp clinical trials/ or clin $\$$ trial\$.ti,ab,rw,sh.

0111 or 2 or 3 or 4 or 5 or 6 or 7 or 8 or 9 or 10

012 (singl $\$$ or doubl $\$$ or tripl $\$$ or trebl $\$$ or blind $\$$ ).ti,ab,rw,sh. or mask $\$ . t w .013 \exp$ placebos/

014 placebo\$.tw.

015 random $\$$.tw.

016 exp research design/

01712 or 13 or 14 or 15 or 16

01811 or 17

019 limit 18 to human

b) combining a) with all of the following search strategies to identify possible RCTs in peripheral vascular diseases. The search for trials relating to peripheral vascular diseases has been divided into a number of strategies because of the large numbers of trials and the diversity of diseases. Each strategy can be run separately to identify potential trials in a specific area (e.g. abdominal aortic aneurysm). 
articles relating to peripheral aneurysms

020 peripheral aneurysm\$.tw.

021 (peripheral adj5 aneurysm\$).tw.

022 iliac aneurysm/

023 iliac aneurysm $\$$.tw.

024 femoral aneurysm $\$$.tw.

025 popliteal aneurysm\$.tw.

026 traumatic aneurysm\$.tw.

02720 or 21 or 22 or 23 or 24 or 25 or 26

02827 and 19

articles relating to arteriovenous fistulae

029 arteriovenous fistula/

030 arteriovenous fistula $\$$.tw.

03129 or 30

03231 and 19

articles relating to polyarteritis nodosa

033 polyarteritis nodosa/

034 polyarteritis nodosa.tw.

03533 or 34

03635 and 19

articles relating to the prevention of pulmonary embolism

037 exp pulmonary embolism/

038 pulmonary embolism.tw.

03937 or 38

040 exp primary prevention/

041 prevention.tw.

042 prophylaxis.tw.

04340 or 41 or 42

04439 and 43

04544 and 19

articles relating to peripheral arterial occlusive diseases

046 peripheral vascular diseases/

047 vascular diseases/

048 arteriosclerosis obliterans /

049 atherosclerosis/

050 arterial occlusive diseases/

051 intermittent claudication/

052 (intermittent adj5 claudica\$).tw.

053 (arteriosclerosis adj5 obliterans).tw.

054 (atherosclerosis adj5 obliterans).tw.

055 exp amputation/

056 (amputat\$).tw.

057 (claudica\$).tw.

058 (peripheral adj5 arteri\$).tw. 
059 (peripheral adj5 occlu\$).tw.

060 (peripheral adi5 vascular).tw.

061 (limb adj5 ischaem\$).tw.

06246 or 47 or 48 or 49 or 50 or 51 or 52 or 53 or 54 or 55 or 56 or 57 or 58 or 59

or 60 or 61

06362 or (peripheral adj5 disease\$).tw.

06463 and 19

articles relating to abdominal aortic aneurysms

065 aortic aneurysm, abdominal/

066 aortic rupture/

067 aorta, abdominal/

068 (abdominal adj5 aneurysm\$).tw.

069 aneurysm, ruptured/

070 (aortic adj 5 aneurysm\$).tw.

071 (abdominal adj5 dilatation\$).tw.

072 (thoracoabdominal adj5 aneurysm).tw.

07365 or 66 or 67 or 68 or 69 or 70 or 71 or 72

07473 and 19

articles relating to vasospastic disorders

075 raynaud's disease/

076 (raynaud\$).tw.

077 (raynaud's adj5 disease\$).tw.

078 (raynaud's adj5 phenomen\$).tw.

079 (digital adj5 vasospasm).tw.

08075 or 76 or 77 or 78 or 79

08180 and 19

articles relating to chronic venous insufficiency and varicose veins

082 varicose veins/

083 venous insufficiency/

084 phlebography/

085 (varicose adj5 vein\$).tw.

086 phlebography.tw.

087 (venous adj5 insufficiency).tw.

088 (chronic venous insufficiency).tw.

08982 or 83 or 84 or 85 or 86 or 87 or 88

09089 and 19

articles relating to vascular surgery

091 vascular surgical procedures/

092 vascular surg $\$$.tw.

093 exp angioplasty/

094 femoral artery/

095 popliteal artery/

096 femoro?popliteal.tw.

09794 or 95 or 96

098 graft.tw. 
09997 and 98

100 revascular\$.tw.

101 bypass.tw.

102 distal.tw.

103 femoro\$.tw.

104 101or102 or103.ti,ab,rw,sh.

105 saphenous vein/

106 reverse.tw.

107105 and 106

108 popliteal.tw.

109101 and 102 and103

11091 or 92 or 93 or 94 or 95 or 96 or 99 or 100 or 104 or 107 or 108 or 109

111110 and 19

articles relating to arterial and mixed ulcers, of the foot and leg

112 leg ulcer/

113 foot ulcer/

114 (arterial adj5 ulcer\$).tw.

115 (leg adj5 ulcer\$).tw.

116 (foot adj5 ulcer\$).tw.

117 (isch?emi\$ adj5 ulcer\$).tw.

118112 or 113 or 114 or 115 or 116 or 117119118 and 19

articles relating to thromboembolism

120 thromboembolism/

121 thrombophlebitis/

122 exp heparin, low-molecular-weight/

123 femoral vein/

124 popliteal vein/

125 (venous adj5 thromb\$).tw.

126 DVT.tw.

127 thromboembolism.tw.

128120 or 121 or 122 or 123 or 124 or 125 or 126 or 127129128 and 19

articles relating to all PVD scope topics

13028 or 32 or 36 or 45 or 64 or 74 or 81 or 90 or 111 or 119 or 129

EMBASE (OVID version) 1980 to 1999 (March)

Mrs Hazel Dunn and Prof Jos Kleijnen have searched EMBASE (OVID version) from 1980-1997. Dr Carol Forbes has searched EMBASE (OVID version) from Jan to Nov 1998. The strategies used were:

a) Identifying possible randomised controlled trials

001 randomization/

002 controlled study/

003 single blind procedure/

004 placebo/ 
005 double blind procedure/

006 clinical trial/

007 crossover procedure/

008 placebo.tw.

009 blind $\$$ fashion.tw.

010 random\$.tw.

011 clinical trial?.tw.

0121 or 2 or 3 or 4 or 5 or 6 or 7 or 8 or 9 or 10 or 11

013 limit 12 to human

b) combining a) with all of the following search strategies to identify possible RCTs in peripheral vascular diseases. The search for trials relating to peripheral vascular diseases has been divided into a number of strategies because of the large numbers of trials and the diversity of diseases. Each strategy can be run separately to identify potential trials in a specific area (e.g. abdominal aortic aneurysm).

articles relating to peripheral arterial occlusive diseases

014 claudica $\$$.tw.

015 (peri\$ adj7 occlusi\$).tw.

016 (occlusi\$ disease and lower extremity).tw.

017 (leg? adj7 isch?em\$).tw.

018 (limb? adj7 isch?em\$).tw.

019 (occlusive adj7 arteriosclerosis).tw.

020 intermittent claudication/

021 claudication/

022 blood vessel occlusion/

023 leg ischemia/

$024 \mathrm{limb}$ ischemia/

025 femoral artery/

026 popliteal artery/

027 iliac artery/

028 superficial femoral artery/

029 internal iliac artery/

030 artery occlusion/

031 occlusi\$.tw.

03225 or 26 or 27 or 28 or 29

03330 or 31

03432 and 33

03514 or 15 or 16 or 17 or 18 or 19 or 20 or 21 or 22 or 23 or 24 or 34

03635 and 13

articles relating to thromboembolism and prevention or pulmonary embolism

037 thromboembolism/

038 thrombophlebitis/

039 (venous adj7 thromb\$).tw.

040 thromboembolism.tw.

041 (pulmonary adj 5 embolism).tw.

042 prevention/

043 prophylaxis/

044 prevention.tw.

045 prophylaxis.tw. 
04642 or 43 or 44 or 45

04741 and 46

048 embolism prevention/

049 thrombosis prevention/

05037 or 38 or 39 or 40 or 47 or 48 or 49

05150 and 13

articles relating to abdominal aortic aneurysms

052 abdominal aorta aneurysm/

053 aorta rupture/

054 abdominal aorta/

055 aneurysm rupture/

056 (abdominal adj5 dilatation).tw.

057 (aortic adj5 aneurysm).tw.

058 (abdominal adj5 aneurysm).tw.

059 (thoracoabdominal adj 5 aneurysm).tw.

06052 or 53 or 54 or 55 or 56 or 57 or 58 or 59

06160 and 13

articles relating to vasospastic disorders

062 raynaud phenomenon/

063 raynaud\$.tw.

064 (raynaud? adj5 disease?).tw.

065 (raynaud? adj5 phenomen\$).tw.

066 (digital adj5 vasospasm\$).tw.

06762 or 63 or 64 or 65 or 66

06867 and 13

articles relating to mixed and arterial ulcers, of the foot and leg

069 foot ulcer/

070 leg ulcer/

071 plantar ulcer/

072 trophic ulcer/

073 (chronic adj5 ulcer?).tw.

074 (isch?em $\$$ adj 5 ulcer?).tw.

075 (arterial adj5 ulcer?).tw.

076 foot ulcer\$.tw.

077 leg ulcer\$.tw.

07869 or 70 or 71 or 72 or 73 or 74 or 75 or 76 or 77

07978 and 13

articles relating to chronic venous insufficiency and varicose veins

080 exp vein insufficiency/

081 leg phlebography/

$082 \mathrm{leg}$ varicosis/

083 (varicose adj 5 vein \$).tw.

084 phlebography.tw.

085 (venous adj 5 insufficiency).tw.

08680 or 81 or 82 or 83 or 84 or 85 
08786 and 13

articles relating to arteriovenous fistulae

088 arteriovenous fistula/

089 arteriovenous fistula $\$$.tw.

09088 or 89

09190 and 13

articles relating to polyarteritis nodosa

092 polyarteritis nodosa/

093 polyarteritis nodosa.tw.

09492 or 93

09594 and 13

articles relating to vascular surgery

096 vascular surgery/

097 vascular surg $\$$.tw.

098 angioplasty/

099 femorofemoral bypass/

100 femoropopliteal bypass/

101 saphenous vein graft/

102 femoral artery/

103 popliteal artery/

104 femoro?popliteal.tw.

105102 or 103 or 104

106 graft.tw.

107105 and 106

108 bypass.tw.

109 distal.tw.

110 femoro $\$$.tw.

111108 and 109 and 110

11296 or 97 or 98 or 99 or 100 or 101 or 107 or 111

113112 and 13

articles relating to peripheral aneurysms

114 peripheral aneurysm\$.tw.

115 (peripheral adj5 aneurysm\$).tw.

116 (femoral adj5 aneurysm\$).tw.

117 (popliteal adj5 aneurysm\$).tw.

118 (iliac adj5 aneurysm\$).tw.

119114 or 115 or 116 or 117 or 118

120119 and 13

articles relating to all PVD scope topics

12136 or 51 or 61 or 68 or 79 or 87 or 91 or 95 or 113 or 120 


\section{Journal Handsearches}

The following 38 core journals relating to peripheral vascular diseases are presently being handsearched, or have been handsearched up until last issue. Up to date information on journal handsearching can be found at URL: www.cochrane.org.

Angiology (1950-1997 - ongoing)

Annals of Vascular Surgery (1986-1998 - ongoing)

Arteriosclerosis (1981-1990)

Arteriosclerosis and Thrombosis (1991-1995)

Arteriosclerosis, Thrombosis and Vascular Biology (1995)

Atherosclerosis (1970-1996)

Circulation (1980-1988)

Clinical and Applied Thrombosis/Haemostasis (1995-1998 - ongoing)

Clinical Haemorheology (1981-1996)

Critical Ischaemia (1990-1995)

European Journal of Vascular and Endovascular Surgery (1994-1998 - ongoing)

Haemostasis (1972-1996 - ongoing)

International Angiology (1982-1998 - ongoing)

International Journal of Angiology (1992-1996 - ongoing)

Journal of Atherosclerosis Research (1961-1969)

Journal of Smoking Related Disorders (1991-1995)

Journal of Thrombosis and Thrombolysis (1994-1996 - ongoing)

Journal of Vascular and Interventional Radiology (1990-1998 - ongoing)

Journal of Vascular Investigation (1995-1997)

Journal of Vascular Medicine and Biology (1989-1994)

Journal of Vascular Nursing (1991-1998 - ongoing)

Journal of Vascular Surgery (1984-1998 - ongoing)

Microvascular Research (1968-1996)

Perfusion (1988-1994)

Phlebology (1986-1998 - ongoing)

Prostaglandins and Medicine (1978-1981)

Prostaglandins Leukotrienes and Medicine (1982-1987)

Prostaglandins Leukotrienes and Essential Fatty Acids (1988-1995)

Regional Anesthesia (1980-1996)

Seminars in Thrombosis and Haemostasis (1974-1998)

Seminars in Vascular Surgery (1988-1998 - ongoing)

Thrombosis et Diathesis Haemorrhagica (1957-1977)

Thrombosis and Haemostasis (1978-1998 - ongoing)

Thrombosis Research (1972- 1997 - ongoing)

Vascular Diseases (1964-1968)

Vascular Medicine (1996-1997)

Vascular Surgery (1967-1998 - ongoing)

VASA (1985-1997)

\section{Conference Proceedings}

The following conference proceedings have been searched, and are displayed according to the year in which the conference was held: 
Venous Forum of the Royal Society of Medicine, Weetwood Hall, Leeds, UK; 16 April 1999. Abstracts

A Symposium of the Venous Forum of the Royal Society of Medicine, Edinburgh, UK; 22-23 October 1998. Epidemiology, Management \& Delivery of Care for Venous Disease - Short Papers, 1998

The Vascular Surgical Society of Great Britain \& Ireland. 31st Annual Conference, 26-28 November 1997. The Royal Lancaster Hotel, London, UK

European Society for Vascular Surgery. XI Annual Meeting. Lisbon, Portugal, 17-20 September, 1997

Fourth International Congress on Essential Fatty Acids \& Eicosanoids. Edinburgh, UK, July 20-24, 1997 (PVD articles only)

XVIth Congress of the International Society on Thrombosis and Haemostasis, Florence, Italy; 6-12 June 1997. Thrombosis and Haemostasis Supplement, June $1997 ; 1-844$

Atherothrombosis and Peripheral Arterial Disease. Boston, USA, June 1, 1997 (PVD articles only)

Cardiovascular Disease Prevention III. London, February 1997

Proceedings of the Second Pacific Vascular Symposium: Advances in Venous Disease. Kamuela, Hawaii, November 1996

Proceedings of the 30th Annual Conference of the Vascular Society of Great Britain and Ireland. Bournemouth International Centre, 20-22 November 1996

European Society for Vascular Surgery 10th Annual Meeting, 12-15 September 1996, Venice, Italy

International Union of Angiology European Chapter's Congress. Budapest, Hungary, May 19-20, 1996

New Aspects of Haemophilia Treatment. Proceedings of the 3rd symposium. Copenhagen, Denmark, September 21-23, 1995. Haemostasis 1996;26 Suppl 1:1-166

Union Internationale de Phlebologie XII World Congress, 3 - 5 September 1995, London, UK. Phlebology '95 1995; 1 and 2 
XVth Congress of the International Society on Thrombosis and Haemostasis. Jerusalem, Israel, June 11-16, 1995. Abstracts.Thrombosis \& Haemostasis 1995 June; 73(6):899-1534

Proceedings of the 64th European Atherosclerosis Society Congress. Utrecht, 10-13 June 1995. Atherosclerosis 1995 December; 118 Suppl:S1-149

64th European Atherosclerosis Society Congress. Utrecht, 10-13 June 1995. Abstracts. Atherosclerosis 1995 June; 115 Suppl:S1-149

International Union of Angiology. London, April, 1995. International Angiology 1995; part 1, Suppl 1

Proceedings of the XII World Congress of the International Union of Phlebology-parallel session entitled medical and socioeconomic impact of venolymphatic disease: benefit of daflon $500 \mathrm{mg}$. London, 1995. Angiology 1997 January; 48(1):21-98

Xth International Symposium on Atherosclerosis. Montreal, 9-14 October 1994. Abstracts. Atherosclerosis 1994 Sep 15;109(1-2):1-354

European Society for Vascular Surgery 8th Annual Meeting, 3-6 September 1994, Berlin, Germany

5th Mediterranean Congress of Angiology and Vascular Surgery. Ajaccio, Corsica, June 20-23, 1994. Abstracts. International Angiology 1994 June; 13 (2 Suppl 1):1-89

13th International Congress on Thrombosis, 10th National SETH Congress, 6th Nursing Workshop. Bilbao, Spain, June 6-10, 1994. Abstracts. Haemostasis 1994;24 Suppl 1:1-410

"EUROCHAP" -94. Angiology. IIIrd meeting of British-Swedish Angiology Societies. Lund, Sweden, May 15-19, 1994. Abstracts. International Angiology 1994 March; 13(1 Suppl 1):1-110

4th International Workshop on Lipid Metabolism. Dijon, France, 15-16 April 1994. Proceedings and abstracts. Atherosclerosis 1994 Oct;110 Suppl:S1-106

International Conference on Vascular Diseases of Lower Limbs. Bari, Italy, November 19-20, 1993. Abstracts. International Angiology 1993 Sep;12(3 Suppl 1):185

European Society for Vascular Surgery 7th Annual Meeting, 22-24 September 1993, Barcelona, Spain

British Hyperlipidaemia Association; 7th Scientific \& Annual General Meeting. Newcastle-upon-Tyne, UK July 8-10, 1993 (PVD articles only)

XIVth International Congress on Thrombosis and Haemostasis, 1993 State of the Art. New York, New York, July 1993. Papers. Thrombosis \& Haemostasis 1993 Jul $1 ; 70(1): 1-232$ 
XIVth Congress of the International Society on Thrombosis and Haemostasis. New York, New York, USA. July 4-9, 1993. Abstracts. Thrombosis \& Haemostasis 1993 Jun 30;69(6):539-1455

The proceedings of the Straub Pacific Health Foundation; Hawaii, June 12-14, 1993

2nd International Joint Workshop on Atherosclerosis, Peripheral Arterial Disease, New Trends in Cardiovascular Therapy. Proceedings. Monte Carlo, January 28-30, 1993. International Angiology 1993 Dec;12(4):326-86

International Congress VI: Endovascular Interventions--Tomorrow's Technology Today. Scottsdale, Arizona, January 27-31, 1993. Abstracts. Angiology 1993 January; 44(1 Suppl):1-30

12th National Congress of the Italian Society for the Study of Haemostasis and Thrombosis in collaboration with the Spanish Group of Haemostasis and Thrombosis. Parma, Italy, September 27 - October 1 1992. Thrombosis Research 1993;70 Suppl 1

European Society for Vascular Surgery 6th Annual Meeting, 2-4 September 1992, Athens, Greece.

Proceedings of the 3rd Novo Nordisk International Symposium on Thrombotic Disorders. Copenhagen, Denmark, September 30-October 1, 1992. Haemostasis 1993 Mar;23 Suppl 1:1-228

Union Internationale de Phlebologie XI World Congress, 30 August - 4 September 1992, Montreal, Canada. Phlebology 92 1992; 1 and 2

Hemostasis in Liver Transplantation. Proceedings of a workshop. Berlin, Germany, August 1992. Seminars in Thrombosis \& Hemostasis 1993;19(3):183-314

Abstracts of the 12th International Congress on Thrombosis. Florence, Italy, May 18-23, 1992. Thrombosis Research 1992;65 Suppl 1

Strategies in endovascular interventions. Proceedings of the International Congress V--Part 2. Scottsdale, Arizona, February 12-16, 1992. Abstracts. Angiology 1992 Mar;43(3 Pt 2):253-79

At the Crossroads of Venous Insufficiency and Hemorrhoidal Disease: Daflon 500 mg. Proceedings of a symposium. Vienna, Austria, January 31, 1992. Angiology 1994 Jun;45(6 Pt 2):493-584

Vascular Symposium:'Angioplasty, Thrombolysis \& Angioscopy - A minimally invasive approach to arterial disease'. Department of Surgery, University of Edinburgh, UK, November 8, 1991

European Society for Vascular Surgery 5th Annual Meeting, 25-27 September 1991, Warsaw, Poland 
XIIIth Congress of the International Society on Thrombosis and Haemostasis. Amsterdam, the Netherlands, 30 June - 6 July 1991. Abstracts. Thrombosis \& Haemostasis 1991 June 5;65(6):645-1366

Low, Molecular Weight Heparins and Related Polysaccharides. Proceedings of an international symposium. Munich, Germany, June 27-28, 1991. Issue dedicated to Gunther Blumel. Seminars in Thrombosis \& Hemostasis 1993;19 Suppl 1:1-247

Development of non-heparin glycosaminoglycans as therapeutic agents: II. A symposium. Chicago. Seminars in Thrombosis \& Hemostasis 1991;17 Suppl 2:137245

Papers from the Peripheral Vascular Surgical Society 1991 meeting. Annals of Vascular Surgery 1992 January; 6(1):1-98

9th National Conference on Thrombosis and Hemostasis. Dallas, Texas, November 1990. Abstracts. Arteriosclerosis 1990 September-October; 10(5):887a-957a

63rd Scientific Sessions of the American Heart Association: Nutrition. Dallas, Texas, November 1990. Abstracts. Arteriosclerosis 1990 September-October; 10(5):872a$886 \mathrm{a}$

44th Annual Meeting. Council on Arteriosclerosis. Dallas, Texas, November 1990. Abstracts. Arteriosclerosis 1990 Sep-Oct;10(5):751a-871a

European Society for Vascular Surgery 4th Annual Meeting, 20-21 September 1990, Rome, Italy

Thrombosis and fibrinolysis: current clinical and therapeutic aspects. Chicago Satellite Symposia of the Xth International Congress of Fibrinolysis. Chicago, August 2-3, 1990. Seminars in Thrombosis \& Hemostasis 1991 October; 17(4):317-476

Cell biology of fibrinolysis. Tenth International Congress on Fibrinolysis. August 1990. Seminars in Thrombosis \& Hemostasis 1991 July; 17(3):159-312

Fifteenth annual meeting of the Peripheral Vascular Surgery Society. June 2, 1990, Los Angeles, California.Annals of Vascular Surgery 1991 Mar;5(2):105-95

Heparin-present and future. Proceedings of a symposium. Florence, March 28-31, 1990. Haemostasis 1990;20 Suppl 1:4-223

Papers from the French Vascular Surgery Society 1990 meeting. Annals of Vascular Surgery 1991 January; 5(1):1-65

10th Congres Modial Union Internationale de Phlebologie. Strasbourg, France, 25-29 August 1989 
XIIth Congress of the International Society on Thrombosis and Haemostasis. Tokyo, Japan, 19-25 August 1989. Abstracts. Thrombosis \& Haemostasis 1989 August 19;62(1):1-647

Clinical significance of fibrinolysis. XIIth congress of the International Society on Thrombosis and Haemostasis. August 1989. Proceedings. Seminars in Thrombosis \& Hemostasis 1990 July; 16(3):203-73

New aspects on hirudin. Proceedings of the 1st International Symposium on Hirudin. Frankfurt, June 23-24, 1989. Haemostasis 1991;21 Suppl 1:1-171

Papers from the French Society for Vascular Surgery--1989 meeting. Annals of Vascular Surgery 1990 March; 4(2):105-70

8th National Conference on Thrombosis and Hemostasis. Washington, D. C., November 1988. Abstracts. Arteriosclerosis 1988 September-October; 8(5):648a$736 a$

42nd annual meeting, Council on Arteriosclerosis, American Society for the Study of Arteriosclerosis. Washington, D.C., November 1988. Abstracts. Arteriosclerosis 1988 September-October; 8(5):549a-647a

Management and clinical use of intravenous nicarpidine. Presented at the 35th annual meeting of the American College of Angiology. Orlando, Florida, October, 1988. Proceedings. Angiology 1990 November; 41(11 Pt 2):977-1022

European Society for Vascular Surgery 2nd Annual Meeting, 14-16 September 1988, Rotterdam, The Netherlands

New pharmacological and clinical properties of the venoactive agent: Venoruton (Paroven). Studies presented at the 1st Mediterranean Congress of Angiology. Corfu, Greece, May 29 to June 3, 1988. Abstracts. International Angiology 1988 OctoberDecember; 7(4 Suppl):1-16

Proceedings of a symposium on Fluxum (low molecular weight heparin): a new prophylactic and therapeutic approach to thromboembolic diseases. Held during the 1st Mediterranean Congress of Angiology. Corfu, Greece, June 2, 1988. International Angiology 1988 July-September; 7(3 Suppl):1-47

Lymphatic system and microcirculation. Proceedings of the Third International Symposium on Daflon 500 held during the First Mediterranean Congress of Angiology. Corfu, Greece, May 31, 1988. International Angiology 1989 OctoberDecember; 8(4 Suppl):1-75

41st annual meeting, Council on Arteriosclerosis, American Society for the Study of Arteriosclerosis. Anaheim, California, November 1987. Abstracts. Arteriosclerosis 1987 September-October; 7(5):491a-563a 
Hypertriglyceridemia, arteriosclerosis, and thrombosis: I. September 21-23, 1987, Washington, D.C. Proceedings of a workshop. Seminars in Thrombosis \& Hemostasis 1988 April; 14(2):137-221

Hypertriglyceridemia, arteriosclerosis, and thrombosis: II. September 21-23, 1987, Washington, D.C. Proceedings. Seminars in Thrombosis \& Hemostasis 1988 July; 14(3):227-92

International Symposium on Familial Hypercholesterolemia. Oslo, Norway, August 28-29, 1987. Proceedings. Arteriosclerosis 1989 January-February; 9(1 Suppl):I1-168

Abstracts of a Satallite Symposia. XIth International Congress on Thrombosis and Haemostasis, 11 July 1987; Brussels, Belgium. Thrombosis Research Supplement VII, 1987 (PVD articles only)

XIth International Congress on Thrombosis and Haemostasis. Brussels, Belgium, 610 July, 1987. Abstracts. Thrombosis \& Haemostasis 1987 July 6;58(1):1-670

Abstracts of a symposium on "Factor VIII/von Willebrand Factor, Platelet Formation, and Function in Health and Disease: A Tribute to Dr. Marion I. Barnhart". Detroit, Michigan, November 21-22, 1986. Seminars in Thrombosis \& Hemostasis 1987 April; 13(2):228-37

Recent advances and new developments in hemostaseology. 4th Congress of the Society on Thrombosis and Haemostasis (GTH). October 12-16, 1986, Dusseldorf. Abstracts. Haemostasis 1986;16 Suppl 5:1-148

Low-molecular-weight heparins. Pharmacology, in vitro and ex vivo effects and clinical results. 4th Congress on Thrombosis and Haemostasis Research. Dusseldorf, October 12-16, 1986. Proceedings. Haemostasis 1988;18 Suppl 3:1-88

32nd Annual Meeting of the International Committee on Thrombosis and Haemostatis, and 9th Congress of the Mediterranean League against Thromboembolic Diseases, Jerusalem, Israel; 1-6 June 1986. Thrombosis Research Supplement VI, 1986 (PVD articles only)

Xth International Congress on Thrombosis and Haemostasis. July 15-19, 1985. Abstracts. Thrombosis \& Haemostasis 1985 July 14;54(1):1-372

Fragmin. Satellite symposium to the 10th congress of the International Society of Thrombosis and Hemostasis. San Diego, July 1985. Haemostasis 1986;16 Suppl 2:171

Low molecular weight heparin and its clinical use. International Enoxaparin symposium. San Diego, July 1985. Haemostasis 1986;16(2):69-188

International Conference on Thrombolysis. Paris, March 15, 1985. Haemostasis 1986;16 Suppl 3:1-152 
Clinical relevance of hemostaseological findings. Abstracts. International symposium and 29th anniversary of the DAB/GTH Saarbrucken, February 20-23, 1985. Haemostasis 1985;15(1):1-86

The use of beta-adrenergic blockers in the treatment of angina and hypertension. A clinical symposium. 31st annual meeting, American College of Angiology and 21st annual meeting, International College of Angiology. San Antonio, Texas, November 6, 1984. Angiology 1986 Mar;37(3 Pt 2):215-62

The clinical scope of disopyramide. A clinical symposium. San Antonio, Texas, November 5, 1984. Proceedings. Angiology 1987 Feb;38(2 Pt 2):165-204

Third meeting of the Working Group on Peripheral Circulation. Palermo, September 6-8, 1984. International Angiology 1985 Oct-Dec;4(4 Suppl):1-197

Proceedings of the defibrotide symposium. 8th International Congress on Thrombosis. Istanbul, June 4-7, 1984. Haemostasis 1986;16 Suppl 1:1-68

7th International Congress on Fibrinolysis. Venezia, March 27-30, 1984. Abstracts. Haemostasis 1984;14(1):1-156

Proceedings of a Symposium , 27-29 September, 1982; Toronto, Canada. Thrombosis Research Supplement IV; Pharmakinetic and Pharmacodynamic Approach to Antithrombotic Therapy, 1983 (PVD articles only)

Symposium of the XII European Conference on Microcirculation, 7 September 1982; Jerusalem, Israel. Thrombosis Research Supplement V; The Endothelial Fibrin Lining, 1983 (PVD articles only)

Seventh International Congress on Thrombosis. Valencia (Spain), October 13-16, 1982. Abstracts. Haemostasis 1982;12(1-2):1-237

Sixth International Congress on Fibrinolysis. Lausanne, July 20-23, 1982. Abstracts. Haemostasis 1982;11 Suppl:1-107

35th annual meeting: Council on Arteriosclerosis, American Society for the Study of Arteriosclerosis, Dallas, Texas, November 1981. Abstracts. Arteriosclerosis 1981 September-October; 1(5):353a-404a

The haemophiliac in the eighties. Workshop III: National haemophilia societies and WFH. Haemostasis 1981;10 Suppl 1:80-108

The haemophiliac in the eighties. Workshop II: Mobilizing support for haemophilia care. Haemostasis 1981;10 Suppl 1:51-79 
34th Annual Meeting. American Society for the Study of Arteriosclerosis. Miami Beach, Florida, November 1980. Abstracts. Arteriosclerosis 1981 Jan-Feb;1(1):52-106

The haemophiliac in the eighties. Proceedings of the 1st International Haemophilia Conference. 14th World Federation of Hemophilia General Assembly Bonn, October 3-7, 1980. Haemostasis 1981;10 Suppl 1:1-308

VIIth International Congress on Thrombosis and Haemostasis, London 15--20 July 1979. Abstracts. Thrombosis \& Haemostasis 1979 July 16;42(1):6-533

Hemorheology and Thrombosis. Proceedings of the United States-Japan Cooperative Seminar, Kobe, Japan, April 28 - May 2; 1975. Thrombosis Research 1975;8 Suppl 2

\section{Efforts To Identify Unpublished Studies}

Members of the Group are aware of the importance of identifying unpublished trials. Reviewers are provided with standard forms which can be sent to those performing trials to obtain details of relevant investigations. Some drug companies, and companies producing diagnostic equipment (e.g. duplex scanners) have also been contacted, and asked whether they are aware of ongoing trials. At the present time it is the responsibility of individual reviewers to contact the drug companies manufacturing the medicines under review.

An offer to supply trial data concerning Ginkgo biloba supplements has been received from Schwabe, and will be followed up shortly.

\section{Other Sources Searched}

An International Venous Taskforce handsearched several journals (not strictly according to the Cochrane criteria) and compiled the results into a series of booklets. These have been handsearched by members of the editorial team.

Mrs Hazel Dunn, has partially searched the ad referendum database published by Info-Med UK; the Cardiovascular Medicine section has been completed to October 1996, and the Rheumatology section for 1996. 
Appendix 4: Inclusion/Exclusion Coding Sheet

Study:

Include/Exclude (circle one)

Inclusion criteria

\begin{tabular}{|l|l|l|l|}
\hline \multicolumn{1}{|c|}{ Criteria } & Yes & \\
\hline $\begin{array}{l}\text { Allocation described as } \\
\text { randomised? }\end{array}$ & & & \\
\hline $\begin{array}{l}\text { Venous ulceration only or } \\
\text { separate arm of factorial trial? }\end{array}$ & & & \\
\hline $\begin{array}{l}\text { Diagnosis derived from } \\
\text { clinical signs/symptoms as a } \\
\text { minimum? }\end{array}$ & & \\
\hline $\begin{array}{l}\text { Intervention=Pentox }+ \\
\text { compression vs placebo }+ \\
\text { compression }\end{array}$ & & & \\
\hline $\begin{array}{l}\text { Primary outcome is objective } \\
\text { outcome assessment? }\end{array}$ & & & \\
\hline
\end{tabular}

Reason for exclusion: 


\section{Appendix 5: Data Extraction Coding Form}

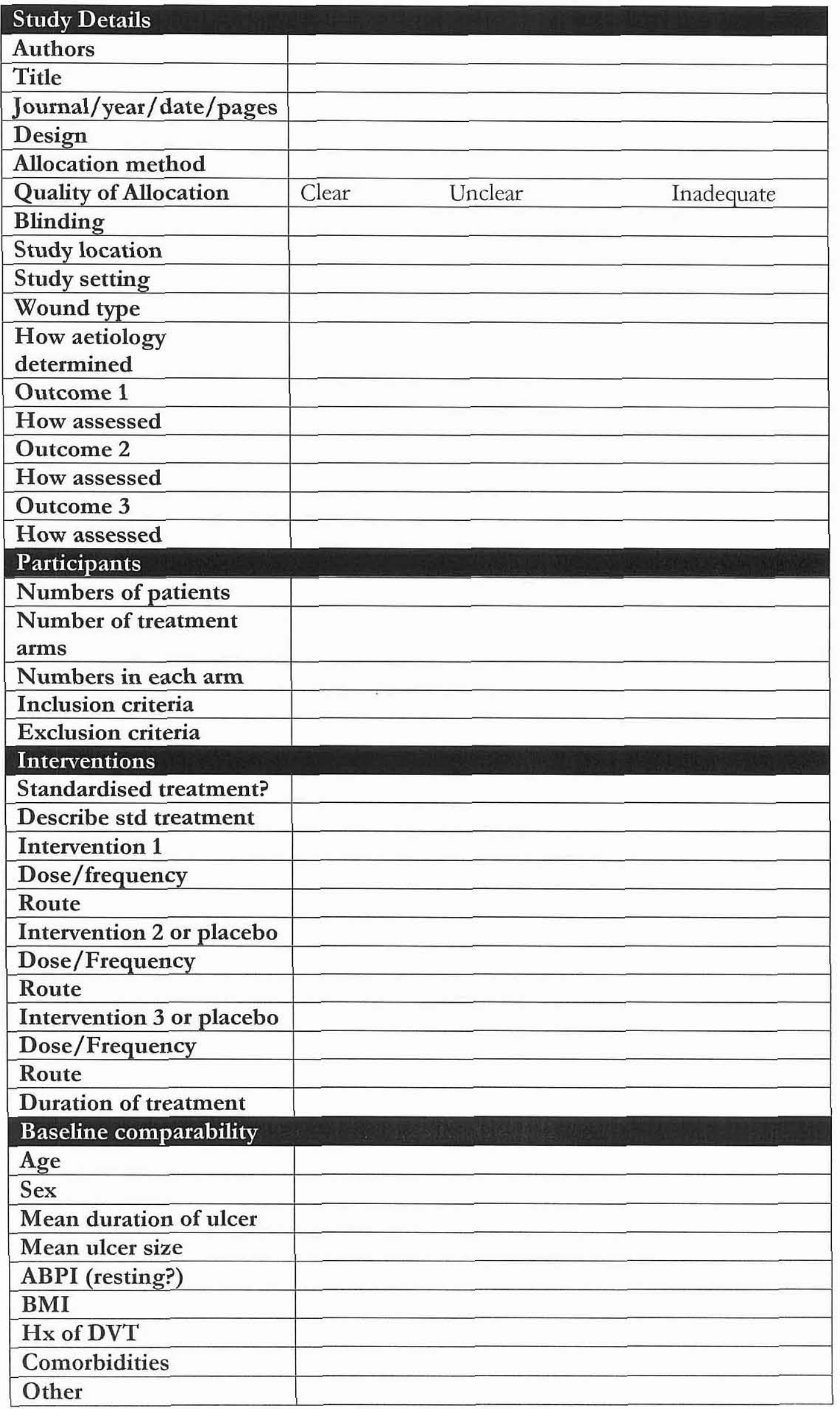




\begin{tabular}{|c|c|c|c|}
\hline \multicolumn{4}{|l|}{ Results } \\
\hline \multirow[t]{2}{*}{ Outcome 1} & (specify) & & \\
\hline & $\mathrm{I} 1=$ & $12=$ & $\mathrm{I} 3=$ \\
\hline \multirow[t]{2}{*}{ Outcome 2} & (specify) & & \\
\hline & $\mathrm{I} 1=$ & $\mathrm{I} 2=$ & $\mathrm{I} 3=$ \\
\hline \multirow[t]{2}{*}{ Outcome 3} & (specify) & & \\
\hline & $\mathrm{I} 1=$ & $\mathrm{I} 2=$ & $\mathrm{I} 3=$ \\
\hline \multicolumn{4}{|l|}{ Side effects } \\
\hline \multicolumn{4}{|l|}{ Any other outcomes } \\
\hline \multicolumn{4}{|l|}{ Withdrawals } \\
\hline \multicolumn{4}{|l|}{ Numbers (total) } \\
\hline \multicolumn{4}{|l|}{ Reason $(\mathrm{n}=)$} \\
\hline \multicolumn{4}{|l|}{ Reason $(\mathrm{n}=)$} \\
\hline \multicolumn{4}{|l|}{ Reason $(\mathrm{n}=)$} \\
\hline \multicolumn{4}{|l|}{ Reason $(n=)$} \\
\hline \multicolumn{4}{|l|}{ Reason $(n=)$} \\
\hline \multicolumn{4}{|l|}{ Comments } \\
\hline \multicolumn{4}{|l|}{$\begin{array}{l}\text { A priori sample size } \\
\text { calculation? }\end{array}$} \\
\hline \multicolumn{4}{|l|}{ Sponsorship? } \\
\hline \multicolumn{4}{|l|}{$\begin{array}{l}\text { Intention to treat } \\
\text { analysis? }\end{array}$} \\
\hline \multicolumn{4}{|l|}{$\begin{array}{l}\text { Any impt outcome } \\
\text { measures omitted? }\end{array}$} \\
\hline Any other comments? & & & \\
\hline
\end{tabular}


Appendix 6: Evidence Tables 
Barbarino (1992) RCT; 2 arm parallel group; double blind; matching placebo
12 participants treated for 1 week as inpatient and 8 weeks as outpatients.

Inclusion criteria: ulcer $>2$

years duration, ulcer resistant to conventional therapy, $A B$ $>0.8$. Exclusion criteria: chronic peripheral obstructive arterial disease; diabetes disorders of circulatory system. Mean age: 1.63 .16 years, 2.64 .83 years. Mean ulcer duration: 1.26 .83 months, 2. 26.33 months.

Mean ulcer size: $1.14 .12 \mathrm{sq} \mathrm{cm}$ ,2. $14.47 \mathrm{sq} \mathrm{cm}$. Mean ABI: 1. $0.84,2.0 .85$.
Group 1: $(\mathrm{n}=6)$ pentoxifylline $1200 \mathrm{mg}$ in 3 divided doses daily, plus 2 layer compression bandaging.

Group 2: $(n=6)$ placebo plus 2 layer compression bandaging.

Treatment duration: 7 days inpatient, 60 days outpatient.
Complete healing: 1. 4/6 $(66 \%), 2.1 / 6(16.67 \%)$. Mean reduction in ulcer size (percent): $1.90 .6 \%, 2.58 .1 \%$ Side effects: $1.2 / 6(33.3 \%)$ $1 / 6(16.67 \%)$. Nil withdrawals.
Each participant tested for responsiveness to treatment prior to randomisation and then underwent washout period for 2 weeks. During hospitalisation, participants received $200 \mathrm{mg}$ intravenous pentoxifylline twice daily in addition to oral dose $(1200 \mathrm{mg})$ or matching placebo.
Colgan, et al. (1990)
RCT; 2 arm parallel group; double blind; matching placebo
80 participants in 4 centres; outpatient clinics. Inclusio criteria: at least 2 month standard treatment with no improvement, $\mathrm{ABI}>0.8$, ulce clinically venous, no contraindication to pentoxifylline. Exclusion criteria: not reported. Mean age: group 1. 71, group 2. 70

Mean duration of ulcer: 1 .

Gmon, 2. 9mon. Mean ulcer size: $1.5 .2 \mathrm{sq} \mathrm{cm}, 2.4 .7 \mathrm{sq} \mathrm{cm}$. Mean ABI: 1. 1.05, 2. 1.06
Group 1: $(\mathrm{n}=38)$ pentoxifylline Number healed at 24 weeks: 1 . $1200 \mathrm{mg}$ in 3 divided doses daily, plus 2 layer compression bandaging. Group 2: $(n=42)$ $23 / 38(60.5 \%), 2.12 / 42$ $(28.6 \%)$. Side effects: $1.17 / 38$ $(44.7 \%), 2.14 / 42(33.3 \%)$.

Withdrawals: $1.3 / 38,2.9 / 42$ Reasons: oedema \& depression, dyspepsia \& diarrhoea, vomiting, skin rash, dizziness \& diarrhoea, celluliti $\&$ pain, headache \& nausea misdiagnosed pemphigoid, poor compliance.
Treatment duration: until weeks
Complete healing $=$ healing of reference ulcer 
Dale, et al. (1999) Factorial RCT; sequential sealed envelop; double blind; matching placebo
200 participants in two

centres; outpatient centres.

Inclusion criteria: Age $>18$;

duration $>2 \mathrm{mon}$; ulcer size $>$

1 diameter; pure venous

aetiology. Exclusion criteria:

MI in past 3 mon;

haemorrhage in past 8 weeks; hypersensitivity to xanthines, pentoxifylline, and drinks containing cola or caffeine systemic treatment with corticosteroids, cytotoxics, naftidrofuryl, oxyrutin, anticoagulents, fibrinolytics or experimental drugs with last 3mon; lumbar sympathectomy within last 3 weeks; presence of right heart failure; sreum creatinine $>180$

micromol/litre; heaptic insufficiency; diabetes;

malignat disease; rheumatoid arthritis or severe connective disorder; limited physical capacity or immobility; infected or gangrenous ulcer; presence of ulcer $<2 \mathrm{mon}$; admission to hospital likely to be required for > 10 days; pregnancy, lactation,

inadequate contraception; life expectancy $<6$ mon. Mean
Multiple interventions balanced between groups i.e. two types of compression evenly balanced between treatment and control groups. Group 1: $(\mathrm{n}=101)$

pentoxifylline $1200 \mathrm{mg}$ in three divided doses daily, plus compression (either elastic single layer or 4 layer bandage). Group 2: ( $\mathrm{n}=99)$ placebo plus compression.

Treatment duration: 24 weeks
Number healed at 24 weeks: 1 . Complete healing $=$ healing of $65 / 101$ (64.3\%), 2. 52/99 all ulcers on reference leg. $(52.5 \%)$. Global side effects: 1

3/101, 2. 3/99. Withdrawals:

11 in each arm. Reasons: side effects; no reason; patient

hospitalised; exclusion criteria discovered after entry; medication omitted by patient $(>14$ days); died. 
age: 1.70 .8 years, 2. 68.1 years.

Median ulcer duration: 1.6

mon, 2. 4mon. Median

maximum ulcer diameter: 1 .

$2.7 \mathrm{sq} \mathrm{cm}, 2.2 .9 \mathrm{sq} \mathrm{cm}$.

Falanga, et al

(1999)
RCT; 3 arm parallel group; double blind; matching placebo
131 participants (129 analysed) in 14 centres; outpatient

clinics. Inclusion criteria: one or more venous ulcers $(>1 \mathrm{~cm}$ in diameter); venous disease; presence of reflux; age 18-90; ulcer duration 2-24mon, absence of significant arteria insufficiency ( $\mathrm{ABI}>0.5$ ); ambulatory; BMI 90-150\% o ideal; able to give informed consent. Exclusion criteria: pregnancy; allergy to xanthines; lumber sympathectomy within last 3 months; HbA1c > 10\%, presence of diabetic or ischaemic ulcers; ulcers with exposed tendon or bone infected ulcers needing systemic antibiotics, history of poor compliance with

treatment. Mean Age: 1. 60.4 years, 2. 58.4 years, 3. 56.4

years. Mean duration of ulcer: 1. 6mon, 2. 6mon, 3. 6mon.
Group 1: $(n=45)$ placebo plus compression (Unna boot \& elastic bandage). Group 2: $(\mathrm{n}=41)$ pentoxifylline $1200 \mathrm{mg}$ in three divided doses daily plus compression. Group 3 $(\mathrm{n}=43)$ pentoxifylline $2400 \mathrm{mg}$ in three divided doses daily, plus compression. Treatment duration: 24 weeks.
Median time to healing: 1.100 days, 2.83 days, 3.71 days.

Number healed at 24 weeks (extrapolated from lifeanalysis): $1.28 / 45(63 \%), 2$. $31 / 41(75 \%), 3.31 / 43(73 \%)$. Global side effects: $1.13 / 45$ $(29 \%), 2.9 / 41(22 \%), 3.15 / 43$ $(35 \%)$. Withdrawals: $(\mathrm{n}=32)$ $10 / 45,2.11 / 41,3.11 / 43$. Reasons: not reported.
Complete healing $=$ healing of all ulcers on reference leg. Intention to treat analysis on all participants who enrolled, received treatment and attended at least one follow-up visit; 129/131 enrolled. Study sponsored by drug's manufacturer. 
Mean ulcer size: $1.9 .63 \mathrm{sq} \mathrm{cm}$

2. $11.43 \mathrm{sq} \mathrm{cm}, 3.7 .72 \mathrm{sq} \mathrm{cm}$.

Mean ABI: 1. 1.1, 2. 1.1, 3. 1.1

Schürmann and

Eberhardt (1986)
RCT; 2 arm parallel group; single blind; placebo
24 participants; setting not

stated. Inclusion criteria no

stated. Exclusion criteria

taking vasoactive drugs. Mean

ulcer size: $1.5 .4 \mathrm{sq} \mathrm{cm}, 2.2 .5$

$\mathrm{sq} \mathrm{cm}$. No other data reported.
Group 1: $(n=12)$ pentoxifylline

$1200 \mathrm{mg}$ daily plus

compression. Group 2: $(n=12)$

placebo plus compression.

Treatment duration: 8 weeks.
Healing at 8 weeks: $1.2 / 12$

$(16 \%), 2.3 / 12(25 \%)$. Mean

1. $2.5 \mathrm{sq} \mathrm{cm}, 2.1 .1 \mathrm{sq} \mathrm{cm}$. Side Blinding not described.

effects: $1.0 / 12,2.2 / 12$. Nil

withdrawals. 


\section{References}

Angelides, N.S., \& Weil von der Ahe, C.A. (1989). Effect of oral pentoxifylline therapy on venous lower extremity ulcers due to Deep Venous Incompetence. Angiology, 40(8), 752-763.

Anning, S.T. (1954). Leg ulcers: their causes and treatment. London: Churchill.

Antman, E.M., Lau, J., Kupelnick, B., Mosteller, F., \& Chalmers, T.C. (1992). A comparison of results of meta-analyses of randomized control trials and recommendations of clinical experts. JAMA, 268(2), 240-248.

Apollonio, A., \& Angeletti, R. (1992). Terapia conservativa delle ulcere flebostatiche (confronto tra pentossifillina defibrotide impiegati per via generale) [Conservative treatment of venous leg ulcers (pentoxifylline vs defibrotide)]. Giornale Italia Angiologia, 12(3), 153-156

Arenas, R., \& Atoche, C. (1988). Postthrombotic leg ulcers: Safety and efficacy of treatment with Pentoxifylline (Double-blind study in 30 patients). Dermatologia Revista Mexicana, 32(2), 34-38.

Bailey, K.R. (1987). Inter-study differences: How should they influence the interpretation and analysis of results? Statistics in Medicine, 6, 351-358.

Baker, S.R., Stacey, M.C., Jopp-McKay, A.G., Hoskin, S.E., \& Thompson, P.J. (1991). Epidemiology of chronic venous ulcers. British Journal of Surgery, 78, 864-867.

Barbarino, C. (1992). Pentoxifylline in the treatment of venous leg ulcers. Current Medical Research and Opinion, 12(9), 547-551.

Beecher, H.K. (1955). The powerful placebo. Journal of the American Medical Association, 159(17), 1602-1606.

Begg, C., Cho, M., Eastwood, S., Horton, R., Moher, D., Olkin, I., Pitkin, R., Rennie, D., Schulz, K.F., Simel, D., \& Stroup, D.F. (1996). Improving the quality of reporting of randomized controlled trials: The CONSORT Statement. JAMA, 276(8), 637-639.

Bosanquet, N. (1992). Costs of venous ulcers: From maintenance therapy to investment programmes. Pblebology, Suppl. 1, 44-46.

Bosanquet, N., Franks, P.J., Brown, D., Straub, J., Harper, D.R., \& Ruckley, C.V. (1995). Cost effectiveness of oxpentifylline in venous ulcer healing. Phlebology, Suppl 1, 36.

Browse, N.L. (1983). Venous ulceration. British Medical Journal, 286, 1920-1922.

Browse, N.L., \& Burnand, K.G. (1982). The cause of venous ulceration. Lancet, $i i$, 243-245. 
Burnard, P., \& Morrison, P. (1994). Nursing research in action: Developing basic skills (2nd ed.). London: MacMillan Press.

Burns, N., \& Grove, S.K. (1987). The practice of nursing research: Conduct, critique and utilization. Philadelphia: WB Saunders.

Callam, M.J., Ruckley, C.V., Harper, D.R., \& Dale, J.J. (1985). Chronic ulceration of the leg: extent of the problem and provision of care. British Medical Journal, 290, 1855-1856.

Callam, M.J., Harper, D.J., Dale, J.J., \& Ruckley, C.V. (1987). Chronic ulcer of the leg: clinical history. British Medical Journal, 294, 1389-1391.

Carr, L., Philips, Z., \& Posnett, J. (1999). Comparative cost-effectiveness of fourlayer compression bandaging in the treatment of venous leg ulceration. Journal of Wound Care, 8(5), 243-248.

Charles, H. (1991). Compression healing of ulcers. Journal of District Nursing, 4, 6-7.

Charles, H. (1995). The impact of leg ulcers on patients' quality of life. Professional Nurse, 10(9), 571-574.

Chase, S.K., Melloni, M., \& Savage, A. (1997). A forever healing: The lived experience of venous ulcer disease. Journal of V ascular Nursing, 15(2), 73-78.

Clarke, M., \& Oxman, A. Cochrane reviewers' handbook 4.0 [updated July 1999]. In Review Manager (RevMan) [Computer program]. Version 4.0. Oxford: The Cochrane Collaboration.

Cochrane, A.L. (1972). Effectiveness and efficiency: Random reflections on bealth services. London: Nuffield Provincial Hospitals Trust.

Cochrane, A.L. (1979). 1931-1971: A critical review, with particular reference to the medical profession, Medicines in the year 2000 (pp. 1-11). London: Office of Health Economics.

Cochrane Collaboration (1999). Review manager version 4.0 (RevMan) [Computer program]. Oxford, England: Update Software.

Cohen, J. (1977). Statistical power analysis for the behavioral sciences (revised ed.). New York: Academic Press.

Coleridge Smith, P.D., Thomas, P., Scurr, J.H., \& Dormandy, J.A. (1988). Causes of venous ulceration: a new hypothesis. British Medical Journal, 296, 1726-1727.

Colgan, M.P. (1990). Oxpentifylline treatment for venous leg ulcers (Letter). BMJ, $300,1725$.

Colgan, M.P., Dormandy, J.A., Jones, P.W., Schraibman, I.G., Shanik, D.G., \& Young, R.A.L. (1990). Oxpentifylline treatment of venous ulcers of the leg. BMJ, 300, 972-975. 
Cook, D.J., Guyatt, G.H., Ryan, G., Clifton, J., Buckingham, L., Willan, A., McIlroy, W., \& Oxman, A.D. (1993). Should unpublished data be included in metaanalyses? JAMA, 269(21), 2749-2753.

Cook, D.J., Sackett, D.L., \& Spitzer, W.O. (1995). Methodologic guidelines for systematic reviews of randomized control trials in health care from the Potsdam Consultation on Meta-analysis. Journal of Clinical Epidemiology, 48(1), 167-171.

Cooper, H., \& Hedges, L.V. (1994). The bandbook of research synthesis. New York: Russell Sage Foundation.

Cooper, H.M. (1982). Scientific guidelines for conducting integrative research reviews. Revien of Educational Research, 52(2), 291-302.

Cooper, H.M., \& Rosenthal, R. (1980). Statistical versus traditional procedures for summarizing research findings. Psychological Bulletin, 87(3), 442-449.

Cornwall, J.V., Dore, C.J., \& Lewis, J.D. (1986). Leg ulcers: epidemiology and aetiology. British Journal of Surgery, 73, 693-696.

Cullum, N., Fletcher, A.W., Nelson, E.A., \& Sheldon, T.A. (1999). Compression bandages and stockings in the treatment of venous ulcers (Cochrane Review). Oxford: Update Software.

Dale, J.J., Callam, M.J., Ruckley, C.V., Harper, D.R., \& Berrey, P.N. (1983). Chronic ulcers of the leg: a study of prevalence in a Scottish community. Health Bulletin, 41, 310-314.

Dale, J.J., Ruckley, C.V., Harper, D.R., Gibson, B., Nelson, E.A., \& Prescott, R.J. (1995). A factorial trial of drugs, dressings and bandages in the treatment of leg ulcers. G.W. Cherry, F. Gottrup, J.C. Lawrence, C.J. Moffatt, \& T.D. Turner (Eds.), 5th European Conference on Advances in Wound Management, Harrowgate, U.K.: MacMillan Magazines Ltd.

Dale, J.J., Ruckley, C.V., Harper, D.R., Gibson, B., Nelson, E.A., \& Prescott, R. (1999). Randomised, double blind placebo controlled trial of pentoxifylline in the treatment of venous leg ulcers. BMJ, 319, 875-878.

Devine, E.C., \& Cook, T.D. (1983). A meta-analytic analysis of effects of psychoeducational interventions on length of postsurgical hospital stay. Nursing Research, 32(5), 267-274.

Dickersin, K., \& Berlin, J.A. (1992). Meta-analysis: State-of-the-science. Epidemiologic Reviews, 14, 154-176.

Dickson, R., \& Entwistle, V. (1996). Systematic reviews: keeping up with research evidence. Nursing Standard, 10(19), 32.

Droogan, J., \& Cullum, N. (1998). Systematic reviews in nursing. International Journal of Nursing Studies, 35, 13-22. 
Duby, T., Hofman, D., Cameron, J., Dobloff-Brown, D., Cherry, G., \& Ryan, T.A. (1993). A randomized trial in the treatment of venous leg ulcers comparing short stretch bandages, four layer bandage system and a long-stretch paste bandage system. Wounds - Compendium of Clinical Research and Practice, 5, 276279.

Egger, M., \& Davey Smith, G. (1995). Misleading meta-analysis. BMJ, 310, 752-754.

Egger, M., \& Davey Smith, G. (1997). Meta-analysis: Potentials and promise. BMJ, 315, 1371-1374.

Egger, M., Zellweger-Zahner, T., Schneider, M., Junker, C., Lengeler, C., \& Antes, G. (1997). Language bias in randomised controlled trials published in English and German. Lancet, 350, 326-329.

Eysenck, H.J. (1952). The effects of psychotherapy: an evaluation. Journal of Consulting Psychology, 16, 319-324.

Eysenck, H. (1978). An exercise in mega-silliness. American Psychologist, 33, 517.

Eysenck, H.J. (1994). Meta-analysis and its problems. BMJ, 309, 789-792.

Eysenck, H.J. (1995). Meta-analysis or best-evidence synthesis? Journal of Evaluation in Clinical Research, 1(1), 29-36.

Falanga, V. (1999). Care of venous leg ulcers. Ostomy/Wound Management, 45(Suppl $1 \mathrm{~A}), 33 \mathrm{~S}-43 \mathrm{~S}$.

Falanga, V., Fujitani, R.M., Diaz, C., Hunter, G., Jorizzo, J., Lawrence, P.F., Lee, Y.B., Menzoian, J.O., Tretbar, L.L., Holloway, G.A., Hoballah, J., Seabrook, G.R., McMillan, D.E., \& Wolf, W. (1999). Systemic treatment of venous leg ulcers with high doses of pentoxifylline: efficacy in a randomized, placebocontrolled trial. Wound Repair Regeneration, 7(4), 208-213.

Feldman, K.A. (1971). Using the work of others: Some obervations on reviewing and integrating. Sociology of Education, 44(Winter), 86-102.

Finney, D.J. (1995). A statistician looks at met-analysis. Journal of Clinical Epidemiology, 48(1), 87-103.

Fleiss, J.L. (1986). Analysis of data from multiclinic trials. Controlled Clinical Trials, 7 , 267-275.

Franks, P.J., Bosanquet, N., Brown, D., Straub, J., Harper, D.R., \& Ruckley, C.V. (1999). Perceived health in a randomised trial of treatment for chronic venous ulceration. European Journal of Vascular and Endovascular Surgery, 17(2), 155-159.

Franks, P.J., \& Moffatt, C.J. (1998). Who suffers most from leg ulceration? Journal of Wound Care, 7(8), 383-385.

Gerbag, Z.B., \& Horwitz, R.I. (1988). Resolving conflicting clinical trials: Guidelines for meta-analysis. Journal of Clinical Epidemiology, 41(5), 503-509. 
Gifford, R.H., \& Feinstein, A.R. (1969). A critique of methodology in studies of anticoagulant therapy for acute myocardial infarction. New England Journal of Medicine, 280, 351-357.

Glass, G.V. (1976). Primary, secondary, and meta-analysis of research. Educational Researcher, 5, 3-8.

Glass, G.V. (1977). Integrating findings: The meta-analysis of research. Research in Education, 5, 351-379.

Hamer, C., Cullum, N.A., \& Roe, B.H. (1994). Patient's perceptions of chronic leg ulcers. Journal of Wound Care, 3, 99-101.

Harkiss, K.J. (1985). Cost analysis of dressing materials used in venous leg ulcers. Pharmacy Journal, 235, 268-269.

Hathaway, D. (1986). Effect of preoperative instruction on postoperative outcomes: A meta-analysis. Nursing Research, 35(5), 269-275.

Hedges, L.V., \& Olkin, I. (1980). Vote counting methods in research synthesis. Psychological Bulletin, 88, 359-369.

Hendricks, W.M., \& Swallow, R.T. (1985). Management of stasis leg ulcers with Unna's boot versus elastic support stockings. Journal of the American Academy of Dermatology, 12, 90-98.

Herdy, C.D.C., Thomaz, J.B., Souza, S.R., Robadey, R.A., \& Rodolfo, R. (1997). Acao da pentoxifilina na cicatrizacao das ulceras de estase: Um estudo clinico-comparativo [Efficacy of pentoxifylline in the healing of venous ulcers: a comparative clinical study]. Arquivos Brasileiros de Medicina, 71(4), 157161.

Hogstel, M.O., \& Sayner, N.C. (1986). Nursing research: An introduction. New York: McGraw Hill.

Hollis, S., \& Campbell, F. (1999). What is meant by intention to treat analysis? Survey of published randomised controlled trials. BMJ, 319, 670-674.

Hunt, M. (1997). How science takes stock: The story of meta-analysis. New York: Russell Sage Foundation.

ISIS-4 Collaborative Group. (1995). ISIS-4: a randomised factorial trial assessing early oral captopril, oral mononitrate, and intravenous magnesium sulphate in 58050 patients with suspected acute myocardial infarction. Lancet, 345, 669685.

Jackson, G.B. (1980). Methods for integrative reviews. Review of Educational Research, 50(3), 438-460.

Jadad, A. (1998). Randomised controlled trials. London: BMJ Books.

Jenick, M. (1989). Meta-analysis in Medicine: Where we are and where we want to go. Journal of Clinical Epidemiology, 42(1), 35-44. 
Kitka, M.J., Schuler, J.J., Meyer, J.P., Durham, J.R., Eldrup-Jorgensen, J., Schwarcz, T.H., \& Flanigan, D.P. (1988). A prospective randomized trial of Unna's boot versus hydroactive dressing in treatment of venous stasis ulcers. Journal of Vascular Surgery, 7, 478-483.

Kylma, J., \& Vehvilainen-Julkunen, K. (1997). Hope in nursing research: a metaanalysis of the ontological and epistemological foundations of research on hope. Journal of Advanced Nursing, 25(2), 364-371.

Lamping, D.L. (1997). Measuring health-related quality of life in venous disease: Practical and scientific considerations. Angiology, 48(1), 51-57.

Lees, T.A., \& Lambert, D. (1992). Prevalence of lower limb ulceration in an urban health district. British Journal of Surgery, 79, 1032-1034.

LeLorier, J., Gregoire, G., Benhaddad, A., Lapierre, J., \& Derderian, F. (1997). Discrepancies between meta-analyses and subsequent large randomized, controlled trials. New England Journal of Medicine, 337(8), 536-542.

Light, R.J., \& Pillemar, D.B. (1984). Summing up: The science of reviewing research. Massachusetts: Harvard University Press.

Light, R.J., \& Smith, P.V. (1971). Accumulating evidence: Procedures for resolving contradictions among different research studies. Harvard Educational Review, 41(4), 429-471.

Lindholm, C., Bjellerup, M., Christensen, O.B., \& Zederfeldt, B. (1993). Quality of life in chronic leg ulcer patients. Acta Dermato Venereologica (Stockholm), 73, 440-443.

LoBiondo-Wood, G., \& Haber, J. (1998). Nursing research: Methods, critical appraisal and utilization (4th ed.). St Louis: Mosby.

Mandel, H. (1936). Racial psychic bistory; a detailed introduction and a systematic review of investigations. Leipzig: Heims.

Mateo, M.A., \& Kirchhoff, K.T. (1991). Conducting and using nursing research in the clinical setting. Baltimore: Williams \& Wilkins.

McGowen, S. (1994). From problem to proposal. In J. Robertson (Ed.), Handbook of clinical nursing research. Melbourne: Churchill Livingstone.

Mirshahi, S., Soria, J., Mirshahi, M., Soria, C., Lenoble, M., Vasmant, D., Cambazard, F., \& Claudy, A. (1995). Expression of elastase and fibrin in venous leg ulcer biopsies: A pilot study of pentoxifylline versus placebo. Journal of Cardiovascular Pharmacology, 25(Suppl. 2), S101-S105.

Moher, D., Jadad, A.R., Nichol, G., Penman, M., Tugwell, P., \& Walsh, S. (1995). Assessing the quality of randomised controlled trials: an annotated bibliography of scales and checklists. Clinical Controlled Trials, 16, 62-73. 
Moher, D., Pham, B., Jones, A., Cook, D.J., Jadad, A.R., Moher, M., Tugwell, P., \& Klassen, T.P. (1998). Does quality of reports of randomised trials affect estimates of intervention efficacy reported in meta-analyses? Lancet, 352, 609613.

Morrell, C.J., Walters, S.J., Dixon, S., Collins, K.A., Brereton, L.M.L., Peters, J., \& Brooker, C.G.D. (1998). Cost effectiveness of community leg ulcer clinics: randomised controlled trial. BMJ, 316, 1487-1491.

Mulrow, C.D. (1987). The medical review article: State of the science. Annals of Internal Medicine, 106, 485-488.

Negus, D. (1995). Leg ulcers: A practical approach to management (2nd ed.). Oxford: Butterworth Heinemann.

Nelson, E.A., (ean2@york.ac.uk). (1999). Fixed effects vs random effects. Email to Andrew Jull (andrewj@ahsl.co.nz).

Nelzén, O., Berqvist, D., \& Lindhargen, A. (1991). Leg ulcer etiology - A crosssectional population study. Journal of V ascular Surgery, 14(4), 557-564.

Nelzén, O., Berqvist, D., Lindhagen, A., \& Hallbrook, T. (1991). Chronic leg ulcers: an underestimated problem in primary health care among elderly patients. Journal of Epidemiology and Community Health, 45, 184-187.

Nelzén, O., Bergqvist, D., \& Lindhagen, A. (1994). Venous and non-venous leg ulcers: clincal history and appearance in a population-study. British Journal of Surgery, 81, 182-187.

NHS Centre for Reviews and Dissemination (1996). Undertaking systematic reviems of research on effectiveness: $C R D$ guidelines for those carrying out or commissioning reviews. York: University of York.

O'Flynn, A. (1982a). Meta-analysis of behavioral intervention effects on weight loss in the obese. Unpublished doctoral dissertation, University of Connecticut.

O'Flynn, A.I. (1982b). Meta-analysis. Nursing Researcher, 31(5), 314-316.

Oxman, A.D., Cook, D.J., \& Guyatt, G.H. (1994). Users' guides to the medical literature VI: How to use an overview. JAMA, 272, 1367-1371.

Pearcey, P.A. (1995). Achieving research-based nursing practice. Journal of Advanced Nursing, 22, 33-39.

Phillips, T., Stanton, B., Provan, A., \& Lew, R. (1994). A study of the impact of leg ulcers on quality of life: Financial, social, psychologic implications. Journal of the American Academy of Dermatology, 31(1), 49-53.

Polit, D.F., \& Hungler, B.P. (1999). Nursing research: Principles and methods (6th ed.). Philadelphia: Lippincott. 
Porter, J.M., Rutherford, R.B., Clagett, G.P., Cranley, J.J., O'Donnell, T.F., Raju, S., Zierler, R.E., Browse, N., \& Nicolaides, A. (1988). Reporting standards in venous disease. Journal of Vascular Surgery, 8(2), 172-181.

Prescott, R.J., Nelson, E.A., Dale, J.J., Harper, D.R., \& Ruckley, C.V. (1998). Design of randomized controlled trials in the treatment of leg ulcers: More answers with fewer patients. Phlebology, 13, 107-112.

Price, P., \& Harding, K. (1995). Quality of life [Letter to the Editor]. Lancet, 346, 445.

Price, P., \& Harding, K. (1996). Measuring health related quality of life in patients with chronic leg ulcers. Wounds, 8, 91-94.

Rennie, D., \& Flanagin, A. (1992). Publication bias - the triumph of hope over experience. JAMA, 267, 411-412.

Robertson, J. (1994). Handbook of clinical nursing research. Melbourne: Churchill Livingstone.

Rosenthal, R. (1978). Combining results of independent studies. Psychological Bulletin, 85(1), 185-193.

Ruckley, C.V. (1997). Socioeconomic impact of chronic venous insufficiency and leg ulcers. Angiology, 48(1), 67-69.

Sackett, D.L., Haynes, R.B., Guyatt, G.H., \& Tugwell, P. (1991). Clinical epidemiology: A basic science for clinical medicine. Boston: Little, Brown \& Company.

Sacks, H.S., Berrier, J., Reitman, D., Ancona-Berk, V.A., \& Chalmers, T.C. (1987). Meta-analyses of randomized controlled trials. New England Journal of Medicine, $316(8), 450-455$.

Savitsky, J.P. (1986). Hemorheology and the therapy of vascular disease. American Pharmacy, 26(8), 38-40.

Scherer, R., Dickersin, K., \& Langenberg, P. (1994). Full publication of results initially presented as abstracts: a meta-analysis. JAMA, 272, 158-162.

Schürmann, W., \& Eberhardt, R. (1986). Wirksamkeit von Pentoxifyllin als Zusatz zu Kompressions- und Lokaltherapie bei Patienten mit Ulcus cruris varicosum/postthromboticum [The efficacy of pentoxifylline added to topical therapy in patients with varicose and postthrombotic ulcers]. Therapiewoche, 36, 2343-2345.

Seaman, C.H.C. (1987). Research methods: Principles, practice and theory for nursing (3rd ed.). Connecticut: Appleton \& Lange.

Shaikh, W., Vayda, E., \& Feldman, E. (1976). A systematic review of the literature on evaluative studies of tonsillectomy and adenoidectomy. Pediatrics, 57(3), 401407.

Slavin, R.E. (1986). Best-evidence synthesis: An alternative to meta-analytic and traditional reviews. Educational Researcher, 15(9), 5-11. 
Slavin, R.E. (1995). Best evidence synthesis: An intelligent alternative to metaanalysis. Journal of Clinical Epidemiology, 48(1), 9-18.

Smeeth, L., Haines, A., \& Ebrahim, S. (1999). Numbers needed to treat derived from meta-analyses - sometimes informative, usually misleading. BMJ, 318, 15481551.

Smith, M.L., \& Glass, G.V. (1977). Meta-analysis of psychotherapy outcome studies. American Psychologist, 32, 752-760.

Spittell, J.A. (1986). Pentoxifylline and vascular disease. Rational Drug Therapentics, 20(11), 4-6.

Stellin, G.P., \& Waxman, K. (1989). Current and potential therapeutic effects of Pentoxifylline. Comprehensive Therapy, 15(5), 11-13.

Stoffers, H.E.J.H., Kester, A.D.M., Kaiser, V., Rinkens, P.E.L.M., Kitslaar, P.J.E.H.M., \& Knotterus, J.A. (1996). The diagnostic value of the measurement of the Ankle-Brachial Systolic Pressure Index in primary health care. Journal of Clinical Epidemiology, 49 (12), 1401-1405

Stranden, E., \& Kroese, A.J. (1995). Venodynamic aspects of the pathophysiology of leg ulcers. In A. Waersted, B. Beermann, O. Westbye, \& K. Strandberg (Eds.), The treatment of venous leg ulcers. Oslo: Norwegian Medicines Control Authority Medical Product Agency.

Sutton, A.J., Jones, D.R., Abrams, K.R., Sheldon, T.A., \& Song, F. (1999). Systematic reviews and meta-analysis: a structured review of the methodological literature. Journal of Health Services Research and Policy, 4(1), 49-55.

Taveggia, T.C. (1974). Resolving research controversy through empirical cumulation. Sociological Methods and Research, 2(4), 395-407.

Thompson, S.G. (1994). Why sources of heterogeneity in meta-analysis should be investigated. BMJ, 309, 1351-1355.

Thompson, S.G., \& Pocock, S.J. (1991). Can meta-analyses be trusted? Lancet, $338(1127-1130)$.

Villar, J., Carroli, G., \& Belizán, J.M. (1995). Predictive ability of meta-analyses of randomised controlled trials. Lancet, 345, 772-776.

Wachter, K.W. (1988). Disturbed by meta-analysis? Science, 241, 1407-1408.

Walshe, C. (1995). Living with a venous ulcer: a descriptive study of patients' experiences. Journal of Advanced Nursing, 22, 1092-1100.

Ward, A., \& Clissold, S.P. (1987). Pentoxifylline: A review of its pharmacodynamic and pharmacokinetic properties, and its therapeutic efficacy. Drugs, 34, 50-97.

Weitgasser, H. (1983). The use of pentoxifylline ('Trental 400') in the treatment of leg ulcers: results of a double blind trial. Pharmatherapentica, 3(Suppl 1), 143-151. 
Widmer, L.K. (1978). Peripheral venous disorders. Basle study III. Bern: Hans Huber.

Wilkinson, E., Butterfield, S., Cooper, S., \& Young, E. (1997). Trial of two bandaging systems for chronic venous ulcers. Journal of Wound Care, 6, 339-340.

Wilson, H.S. (1989). Research in nursing (2nd ed.). California: Addison-Wesley.

Wolf, F.M. (1986). Meta-analysis: Quantitative methods for research synthesis. California: Sage Publications.

Yusuf, S., Koon, T., \& Woods, K. (1993). Intravenous magnesium in acute myocardial infarction: An effective, safe, simple and inexpensive intervention. Circulation, 87, 2043-2046. 